\title{
Immune landscapes predict chemotherapy resistance and immunotherapy response in acute myeloid leukemia
}

\author{
Jayakumar Vadakekolathu', Mark D. Minden², Tressa Hood ${ }^{3}$, Sarah E. Church ${ }^{3}$, Stephen
} Reeder $^{1}$, Heidi Altmann ${ }^{4}$, Amy H. Sullivan ${ }^{3}$, Elena J. Viboch ${ }^{3}$, Tasleema Patel ${ }^{5}$, Narmin Ibrahimova², Sarah E. Warren ${ }^{3}$, Andrea Arruda², Yan Liang ${ }^{3}$, Thomas H. Smith ${ }^{3}$, Gemma A. Foulds $^{1}$, Michael D. Bailey ${ }^{3}$, James Gowen-MacDonald ${ }^{3}$, John Muth ${ }^{6}$, Marc Schmitz ${ }^{7,8,9}$, Alessandra Cesano ${ }^{3}$, A. Graham Pockley ${ }^{1,10}$, Peter J.M. Valk ${ }^{11}$, Bob Löwenberg ${ }^{11}$, Martin Bornhäuser $^{4,8,9}$, Sarah K. Tasian ${ }^{5}$, Michael P. Rettig ${ }^{12}$, Jan Davidson-Moncada ${ }^{6}$, John F. DiPersio $^{12}$, Sergio Rutella ${ }^{1,10, *}$

\footnotetext{
1John van Geest Cancer Research Centre, Nottingham Trent University, Nottingham, NG11 8NS, United Kingdom

${ }^{2}$ Division of Medical Oncology and Hematology, Princess Margaret Cancer Centre, Toronto, ON M5G 2C1, Canada

${ }^{3}$ NanoString Technologies Inc., Seattle, WA 98109, United States of America

${ }^{4}$ Department of Medicine, Universitätsklinikum Carl Gustav Carus, 01307 Dresden, Germany ${ }^{5}$ Department of Pediatrics, Division of Oncology and Centre for Childhood Cancer Research, Children's Hospital of Philadelphia and University of Pennsylvania School of Medicine, PA 19104, United States of America

${ }^{6}$ MacroGenics Inc., Rockville, MD 20850, United States of America

${ }^{7}$ Institute of Immunology, Faculty of Medicine Carl Gustav Carus, Technische Universität Dresden, 01307 Dresden, Germany

${ }^{8}$ National Center for Tumor Diseases (NCT), Partner Site Dresden, 01307 Dresden, Germany ${ }^{9}$ German Cancer Consortium (DKTK), Partner Site Dresden, and German Cancer Research Center (DKFZ), 69120 Heidelberg, Germany

${ }^{10}$ Centre for Health, Ageing and Understanding Disease (CHAUD), Nottingham Trent University, Nottingham, NG11 8NS, United Kingdom

${ }^{11}$ Department of Hematology, Erasmus University Medical Centre, 3000CA Rotterdam, Netherlands

${ }^{12}$ Division of Oncology, Department of Internal Medicine, Washington University in St. Louis, St. Louis, MO 63110, United States of America
}

*To whom correspondence should be addressed:

E-mail: sergio.rutella@ntu.ac.uk

Open Researcher and Contributor Identifier (ORCID) ID: 0000-0003-1970-7375

One Sentence Summary: Dissecting the immune architecture of AML and its influence on therapeutic response identifies patients for whom immunotherapy will be most beneficial. 
Abstract: Acute myeloid leukemia $(A M L)$ is a molecularly and clinically heterogeneous hematological malignancy. Although immunotherapy may be an attractive modality to exploit in patients with AML, the ability to predict the groups of patients and the types of cancer that will respond to immune targeting remains limited. This study dissected the complexity of the immune architecture of $A M L$ at high resolution and assessed its influence on therapeutic response. Using 442 primary bone marrow samples from three independent cohorts of children and adults with AML, we defined immune-infiltrated and immune-depleted disease classes and revealed critical differences in immune gene expression across age groups and molecular disease subtypes. Importantly, interferon (IFN)- $\gamma$-related mRNA profiles were predictive for both chemotherapy resistance and response of primary refractory/relapsed AML to flotetuzumab immunotherapy. Our compendium of microenvironmental gene and protein profiles provides insights into the immuno-biology of AML and could inform the delivery of personalized immunotherapies to IFN- $\gamma$-dominant AML subtypes. 


\section{Introduction}

Acute myeloid leukemia (AML) is a malignant disorder characterized by the accumulation of myeloblasts in the bone marrow (BM) and blood (1). The discovery of the genomic landscape of AML, including the identification of targetable mutations (2), has propelled the development of novel anti-leukemic agents and is enabling disease classification and patient stratification into risk groups (3). Despite success in many areas, AML is cured in only $35-40 \%$ of patients $<60$ years of age and in $5-15 \%$ of patients $>60$ years of age. While chemotherapy resistance is common, the majority of patients die of disease relapse. Investigation of new molecularlytargeted and immuno-modulatory agents therefore remains a high priority for both children and adults (4).

Tumor phenotypes are dictated not only by the neoplastic cell component, but also by the immunologic milieu within the tumor microenvironment (TME), which is equipped to subvert host immune responses and hamper effector T-cell function (5). In silico approaches have been instrumental for the identification of immunogenomic features with therapeutic and prognostic implications. In solid tumors, six immune subtypes have been described: wound healing, interferon (IFN)- $\gamma$-dominant, inflammatory, lymphocyte-depleted, immunologically quiet, and transforming growth factor (TGF)- $\beta$-dominant (6). These are characterized by differences in macrophage or lymphocyte signatures, T helper type (Th)-1 to Th2 cell ratio, extent of intratumoral heterogeneity and neoantigen load, aneuploidy, cell proliferation, expression of immunomodulatory genes, and patient survival (6).

Although immunotherapy may be an attractive modality to exploit in patients with AML (7), the ability to predict the groups of patients and the forms of leukemia that will respond to immune targeting remains limited (8-11). Clinical studies in patients with solid tumors have shown that responses to anti-programmed cell death 1 (PD-1)/programmed death ligand 1 (PD-L1)targeted immunotherapy occur most often in individuals with immune-inflamed lesions that are characterized by pre-existing $\mathrm{CD} 8^{+} \mathrm{T}$-cell responses, release of pro-inflammatory and effector cytokines (12-14), and an augmented T-cell receptor (TCR) clonal diversity pre-treatment (15, 
16). The T-cell inflamed gene expression profile (GEP) is a measure of IFN- $\gamma$-responsive genes that are related to adaptive immune resistance (AIR) mechanisms of immune escape such as indoleamine 2,3-dioxygenase-1 (IDO1) and PD-L1 (17), and is predictive of clinical benefit with pembrolizumab immunotherapy $(18,19)$. Although IFN- $\gamma$ plays a critical role in eliciting anti-tumor T-cell activity and enabling tumor rejection, prolonged IFN- $\gamma$ signaling under conditions of persistent antigen exposure has been shown to activate a PD-L1-independent, STAT1-driven multigenic program which confers resistance to radiotherapy and anti-cytotoxic T-lymphocyte antigen 4 (CTLA-4) immunotherapy in mouse models of melanoma (20). Herein, we used targeted immune gene expression profiling (IGEP) and spatially resolved multiplexed digital spatial profiling (DSP) for the high-dimensional analysis of the immunological contexture of a broad collection of BM samples from patients with AML and for the identification of molecular determinants of immunotherapeutic benefit. We reveal unifying immune features and critical differences that define classes and subclasses of TMEs and deliver predictions of chemotherapy resistance, survival and immunotherapy response that are beyond the current capabilities of single molecular markers. 


\section{Results}

Targeted immune gene expression profiling (IGEP) identifies immune subtypes of $A M L$

We first analyzed unfractionated, archival BM samples from treatment-naïve patients with nonpromyelocytic AML (PMCC discovery series; $n=290$ cases; Table 1) (21). We derived immune scores from mRNA expression, similar to those of previous publications, and devised an RNAbased, quantitative metric of immune infiltration $(22,23)$. As shown in Fig. S1A-B, patients with adverse cytogenetic features exhibited a shorter relapse-free survival (RFS) and overall survival (OS) compared with patients with intermediate and favorable cytogenetic risk, thus confirming the overall performance of well-established European Leukemia-Net (ELN) categories (24). A Pearson correlation matrix of immune gene sets allowed us to identify coexpression patterns of pre-defined immune cell types and immune biological activities. Immune signature modules in pre-treatment BM samples reflected the co-expression of genes associated with 1) type I and type II IFN biology, 2) adaptive immune responses, and 3) myeloid cell abundance (macrophages, neutrophils and dendritic cells [DCs]); Fig. 1A). We then computed the sum of the individual scores in each signature module in Fig. 1A and generated three immune scores (IFN-dominant, adaptive, and myeloid) which individually separated AML cases according to high and low expression values (Fig. S1C). Specifically, the IFN-dominant gene module was calculated as the sum of IFN- $\gamma$ signaling, IFN downstream, immunoproteasome, myeloid inflammation, inflammatory chemokine, IL-10, MAGEs, $P D-L 1$ and $P D-L 2$ scores. When considered in aggregate, IFN-dominant, adaptive, and myeloid scores dichotomized BM samples into two immune subtypes, herein termed immune-infiltrated and immune-depleted (Fig. 1B and Fig. S1D-E) (25), which expressed comparable amounts of leukemia-associated antigens CD34, CD123 (IL3RA) and CD117 $(K I T)$. This observation suggests that targeted IGEP of bulk BM specimens largely captured elements of the immunological TME rather than features of the tumor cell compartment (Fig. S1F).

As shown in Fig. 1C, AML cases with immune-infiltrated profiles had higher expression of IFNstimulated genes and T-cell recruiting factors (STAT1, CXCL10, IRF1), T-cell markers and 
cytolytic effectors (CD8A, CD8B, GZMB, PRF1), counter-regulatory immune checkpoints and immunotherapy drug targets (IDO1, CTLA4, PD-L1 and BTLA), and molecules involved in antigen processing and presentation (TAP1, TAP2, HLA-A, HLA-B and HLA-C). Conceivably, high T-cell infiltration and expression of MHC and PD-L1 in the immune-infiltrated AML subtype reflected a pre-existing IFN- $\gamma$-driven adaptive immune response. This type of response has previously been associated with suppressed anti-tumor immune reactivity (13, $26)$, but also with immunotherapy responses in patients with solid tumors $(9,18,27)$ and $A M L$ (11). STAT1, a central component of the IFN- $\gamma$ signaling pathway and predictor of response to immune checkpoint blockade (28), was more strongly correlated with the presence of T-cell inhibitory receptors TNFRSF14 (a ligand for the immunoglobulin superfamily members BTLA and CD160), PD-L1, HAVCR2 (Tim-3) and LAG-3, and with IFN-stimulated genes MX1, IFIT1 and IRF1 in the immune-infiltrated relative to the immune-depleted subtype, consistent with their coordinated regulation in an inflamed TME (Fig. 1D).

Highly multiplexed digital spatial profiling (DSP) reveals distinct T-cell neighbors in immuneinfiltrated and immune-depleted $A M L$

Transcriptomic data do not provide information on spatial relationships of tumor-infiltrating immune cells within the TME. Therefore, we used GeoMx DSP to characterize the expression of 31 immuno-oncology $(\mathrm{IO})$ proteins in 10 randomly selected, fresh frozen paraffin embedded (FFPE) BM biopsies from treatment-naïve patients with AML (SAL cohort) with varying degrees of T-cell infiltration (Fig. S2 and Fig. S3). We selected 24 geometric regions of interest (ROIs) per BM sample using fluorescent anti-CD3 (visualization marker for T-cell-rich ROIs) and anti-CD123 antibodies (visualization marker for myeloid blast-rich ROls; Fig. 2A) (29). T-cell gene expression scores (calculated as detailed in Fig. S4A) correlated with DSP CD3 protein status (Fig. S4B). Furthermore, mRNA and proteins for B cells $(P=0.0026)$, monocytes $(P=0.0156)$ and $\mathrm{Bcl}-2(P=0.0139)$ significantly and positively correlated, serving as a validation for the mRNA-based immune scores (Fig. S4C). 
We then asked whether CD3-rich and CD3-poor BM samples (defined by a median split of barcode counts) differed in terms of co-localization patterns of relevant IO proteins. As shown in Fig. S4D, CD3 ${ }^{+}$T-cells in immune-infiltrated biopsies co-localized with B cells, antigen processing and presentation-related proteins ( $\beta 2$-microglobulin), negative immune checkpoint $\mathrm{B} 7-\mathrm{H} 3$ and $\beta$-catenin. In contrast, $\mathrm{CD}^{+} \mathrm{T}$-cells in immune-depleted biopsies co-localized with markers of immunological memory (CD45RO) and T-cell exhaustion (PD-1). We next assigned each ROI to either the T-cell-rich (CD3 "hotspot") or the T-cell-poor category, using the top and bottom quartile of CD3 barcode counts (Fig. S5A). When compared to T-cell-poor ROIs, CD3 hotspots showed higher protein expression of CD8, $\beta 2$-microglobulin, B7-H3, PD1, total and phosphorylated STAT3, CD14 and CD19 (Fig. S5B). As shown in Fig. 2B, PTEN positively correlated with CD8 and GZMB. Furthermore, baseline expression of PD-L1, Bcl-2, VISTA, Ki-67 and FoxP3 was significantly lower in patients who achieved remission in response to induction chemotherapy compared to patients with primary induction failure (Fig. S5C).

When analyzing overall protein expression patterns (10 samples $\times 24$ ROls per sample $\times 31$ proteins $=7,440$ data points), we identified four protein signatures (SIG), which were then further assessed in silico for correlations with clinical-biological disease characteristics and potential prognostic value in The Cancer Genome Atlas (TCGA)-AML cases (162 sequenced AML samples with putative copy-number alterations, mutations and mRNA expression zscores [threshold \pm 2.0 ]). Abnormalities in SIG1, SIG2 and SIG4 genes (blue boxes in Fig. 2C) did not correlate with specific disease characteristics or clinical outcomes. In contrast, mRNA up-regulation, gene amplification, deep deletion and mis-sense mutations in SIG3 genes, which were detected in $26 \%$ of TCGA-AML cases as mutually exclusive genomic events (Fig. 2D), significantly correlated with TP53 mutation status, an established adverse prognosticator in AML ( $P$ value from mutation enrichment analysis=0.0285). SIG3 were enriched for gene ontology (GO) biological processes related to T-cell lineage commitment, positive T-cell selection and T-cell homeostasis (Fig. 2E). De-regulation in SIG3 genes, which included PD- 
L1, FoxP3, GZMB, PTEN and BCL2, were predominantly observed in patients with immuneinfiltrated mRNA profiles (Fig. 2F) and correlated with higher number of mutations $(P=0.021)$ and with adverse ELN cytogenetic features $\left(\chi^{2}=25.03 ; P<0.001\right)$, but not with other disease characteristics at presentation, including white blood cell (WBC) count, and percentage of AML blasts in pre-treatment blood and BM samples. Finally, patients with abnormalities in SIG3 genes experienced poor clinical outcomes, as shown by the significantly lower RFS and OS rates (Fig. 2G). Although the mutually exclusive pattern of SIG3 genomic abnormalities would suggest that the altered genes are linked in a common biological process or pathway (30), GZMB and FoxP3 were the only SIG3 genes that individually predicted shorter OS (Fig. S6). Collectively, highly multiplexed in situ detection of 10 proteins highlights critical differences in T-cell infiltrated versus T-cell depleted AML subtypes and identifies protein signatures with prognostic potential in T-cell infiltrated pre-treatment samples.

Interactions between immune subgroups, common cytogenetic alterations and clinical factors We next correlated immune signature scores with clinical and demographic factors, including WBC count and blast cell count at diagnosis, ELN cytogenetic category (available in 249 cases from the PMCC discovery series) and patient age. Leukemia burden was significantly lower in the immune-infiltrated AML subtype (median WBC count at diagnosis $P<0.0001$; median percentage of BM blasts $P<0.0001$; and median number of AML blasts per $\mu \mathrm{L}$ of blood $P<$ 0.0001; Fig. S7A-C). Immune signature scores were not correlated with the ELN cytogenetic risk category when considered individually (Fig. S7D). Finally, patients with high immune infiltration tended to be of a more advanced age at diagnosis compared with patients with low immune infiltration $(P<0.0001$; Fig. S7E).

Immune subtypes improve survival prediction

The activation of immune pathways has context-dependent prognostic impact that differs between tumor types (6). We next assessed the ability of the immune subtype to refine the 
accuracy of outcome prediction separately for each ELN cytogenetic risk category. Among patients with favorable risk, RFS and OS times were significantly longer in individuals with an immune-infiltrated TME (Fig. 3A). In contrast, clinical outcomes in ELN adverse risk cases were worse in individuals with an immune-infiltrated TME (Fig. 3A). Interestingly, CD8 exhaustion scores and PD1 scores were significantly higher in patients with ELN adverse risk compared with ELN favorable risk (Fig. 3B), suggesting that leukemia progression may be sustained by tumor cell-extrinsic (immune) mechanisms in patients with more aggressive disease. We also observed that the ELN classifier assisted outcome prediction only in the immune-infiltrated subtype (Fig. 3C), allowing the identification of patient subgroups with excellent survival estimates ( $87.5 \%$ RFS and $77.8 \%$ OS) or with very unsatisfactory outcomes [10.4\% RFS (log-rank $\left.\chi^{2}=15.07 ; P<0.0001\right)$ and $7.2 \%$ OS (log-rank $\left.\left.\chi^{2}=25.75 ; P<0.0001\right)\right]$. Unexpectedly, our immunological classifier was unable to stratify survival in patients with intermediate ELN risk. ELN intermediate patients with NPM1 mutation and FLT3-ITD information (available in 100 cases) were then subclassified into molecular low risk (NPM1 mutations without FLT3-ITD), molecular intermediate risk (NPM1 wild-type without FLT3-ITD or with low FLT3-ITD allelic ratio) and molecular high risk cases (NPM1 wild-type with high FLT3-ITD allelic ratio) (31). As shown in Fig. 3D, the molecular classifier separated survival both in patients with immune-infiltrated AML and in those with an immune-depleted TME. The inclusion of immune gene signatures also improved the ability of NPM1 and FLT3-ITD mutation status to predict survival in multivariate logistic regression models relative to molecular risk alone (AUROC $=0.938$ versus 0.765 ; Fig. $3 E$ ). Specifically, the IFN downstream score (Wald $\chi^{2}=4.1 ; P=0.043$ ), CD8 score (Wald $\chi^{2}=4.2 ; P=0.04$ ), exhausted $\mathrm{CD}^{+} \mathrm{T}$-cell score (Wald $\left.\chi^{2}=4.4 ; P=0.037\right), I D O 1$ score (Wald $\left.\chi^{2}=4.01 ; P=0.045\right)$ and CTLA4 score (Wald $\chi^{2}=5.2 ; P=0.022$ ) significantly contributed to the model.

By performing Cox proportional hazards regression, we also discovered a set of 21 differentially expressed $(\mathrm{DE})$ immune genes [false discovery rate $(F D R)<0.05$ ] between the favorable and adverse-risk ELN category (Fig. 4A and Table S1). This gene set was highly 
expressed in immune-infiltrated AMLs (Fig. 4B) and separated ELN intermediate patients into subgroups with low and high gene expression values, with the former being closely similar to ELN favorable-risk patients while the latter resembled ELN adverse-risk patients. The 21-gene classifier exhibited enrichment of gene ontologies (GO) and pathways related to T-cell activation, TCR downstream signaling and regulation of cytokine production (Fig. 4C). Importantly, RFS and OS estimates were significantly worse for intermediate-risk patients with high versus low expression of the $21 \mathrm{DE}$ genes (Fig. 4D). This finding was validated in silico using transcriptomic data from two independent AML cohorts (TCGA [Fig. 4E] and HOVON [Fig. 4F]).

Finally, we used informative censoring to evaluate the impact of the immune subtypes on outcome whilst excluding potential clinical benefit from graft-versus-leukemia (GVL) effects after allogeneic HSCT. As shown in Fig. S8A and compared to all patients, survival differences remained evident when patients were censored on the date of allogeneic HSCT, suggesting that immune gene profiles largely predicted outcome after conventional chemotherapy. A competing risks regression analysis in which allogeneic HSCT was treated as a potential confounder $(32,33)$ confirmed that the IFN-dominant gene module, but not the adaptive and myeloid gene modules, predicted for shorter OS (Table S2). We also assessed the potential impact of the immune subtype on post-transplantation outcomes. Causes of death were not different in patients with immune-infiltrated and immune-depleted AML (Table S3). In contrast to patients with immune-depleted $A M L$, individuals with immune-infiltrated $A M L$ benefited from a more profound GVL effect after allogeneic HSCT, as suggested by a significantly longer median OS time (31.9 months) compared with patients receiving chemotherapy only (18.9 months, log-rank $P=0.018$ ). Patients with immune-infiltrated AML and adverse ELN features derived the greatest benefit from allogeneic HSCT (28\% versus $3 \%$ survival rate after chemotherapy with or without allogeneic HSCT, respectively; Fig. S8B-C).

Immune landscapes stratify AML patients in independent validation sets and differ across age groups and disease stages 
$A M L$ is a disease with age-dependent biological specificities $(34,35)$. Furthermore, pediatric AML are inherently of low immunogenicity and are therefore less likely to respond to singleagent checkpoint inhibition (36). To characterize the immunological landscape of AML across age groups and longitudinally in patients who initially achieve complete remission (CR) and then experience disease recurrence, we profiled BM samples from a pediatric (CHOP series, $\mathrm{n}=34$ cases, $61 \mathrm{BM}$ specimens in total) and an adult AML cohort (SAL series, $n=46$ cases, 91 BM specimens in total). In line with findings in the PMCC discovery series, we identified IFNdominant, adaptive and myeloid mRNA profiles (Fig. S9A-D) which individually separated AML cases according to high and low expression values and, when considered in aggregate, dichotomized AML cases into an immune-infiltrated and immune-depleted subtype (Fig. S9EF). As summarized in Fig. $\mathbf{5 A}$, comparison between children and adults with AML revealed a set of DE immune genes involved with cytokine and chemokine signaling, as indicated by GO (Fig. S10A and Table S4) and protein interaction network analysis (Fig. S10B). Specifically, genes encoding pro-inflammatory and pro-angiogenic cytokines and chemokines, including IL8, CCL3L1, CCL3 and CXCL2, were more expressed in adult AML relative to childhood AML (Fig. 5A). The tumor inflammation signature (TIS) score and the IFN- $\gamma$ score were significantly higher in elderly patients ( $>60$ years of age) relative to younger adults ( $<60$ years of age) and to children aged less that 18 years (Fig. S10C).

When comparing matched BM samples from a subset of adult patients in the SAL series $(n=21)$ at the time of diagnosis and achievement of CR after induction chemotherapy (Fig. 5B and Fig. S11), we identified a set of DE genes that were enriched for GO biological processes related to immune responses, apoptosis, drug resistance, transcriptional mis-regulation, and cell surface receptor/cytokine-mediated signaling (Table S5). As shown in Fig. 5B, FLT3, CD99, and milk-fat globule EGF-8 (MGFE8), which have previously been implicated in cancer stem cell self-renewal (37), were lower in AML cases with $C R$, serving as a data reliability check. In agreement with recently published observations (38), CTLA4 expression was higher in CR relative to disease onset, with concomitant down-regulation of CD244 coinhibitory molecule, suggesting the occurrence of T-cell activation after treatment (Fig. S10D). 
Furthermore, immune genes significantly associated with relapsed AML (SAL series) largely captured $\mathrm{CD}^{+} \mathrm{T}$-cell infiltration, elements of T-cell biology, including TCR downstream signaling (CD8A, CD5, CD3z [CD247]), leukocyte differentiation and immune regulation (Fig. 5C and Table S6). The increased expression of surrogate markers of terminal T-cell differentiation, senescence and exhaustion [TBX21 (T-bet) $(39,40), T I G I T, K L R D 1$ (38) and KLRF1] in relapsed $\mathrm{AML}$ suggests that $\mathrm{BM}$-infiltrating cytotoxic $\mathrm{T}$ cells may fail to restrain leukemia growth (Fig. S10E). The DE genes between patient subgroups with newly diagnosed, CR and relapsed AML in the SAL cohort, and between childhood and adult cases, were largely non-overlapping, as shown in Fig. 5D.

Upon activation with a polyclonal stimulus, intracellular cytokine staining of BM suspensions showed higher concomitant production of IFN- $\gamma$ and TNF- $\alpha$ by microenvironmental $\mathrm{CD} 4^{+}$and $\mathrm{CD}^{+} \mathrm{T}$ lymphocytes in the immune-infiltrated compared with the immune-depleted subgroup $\left(P=0.0273\right.$; Fig. S12A-D). Polyfunctional IFN- $\gamma^{+} T N F-\alpha^{+}$T cells were significantly reduced in remissional BM samples compared with diagnostic and relapse BMs $(P=0.0257)$ and were particularly low in patients with documented minimal residual disease (MRD) negativity (Fig. S12E).

\section{IFN-related gene sets improve the prediction of therapy resistance}

We then asked whether the IFN-dominant gene module may assist the prediction of therapeutic resistance, which we empirically defined as failure to achieve CR in patients who survived at least 28 days (primary refractory $A M L$ ) or as early relapse ( $<3$ months) after achieving $\mathrm{CR}$, as previously published by others (41). When patients in the PMCC cohort were dichotomized based on higher or lower than median IFN scores, a higher percentage of patients with primary refractory disease was observed in the IFN-score ${ }^{\text {high }}$ AML cases $(65.4 \%$ versus $34.6 \% ; P=0.0022$, Fisher's exact test), suggesting that transcriptional programs orchestrated by microenvironmental IFN- $\gamma$ might render $\mathrm{AML}$ blasts resistant to chemotherapeutic agents $(20,42)$. In contrast, the frequency of primary refractory cases was 
not different when comparing patients with higher or lower than median adaptive module scores $(29.6 \%$ versus $25.4 \% ; P=\mathrm{NS})$ and myeloid module scores $(26.7 \%$ versus $28.1 \%$; $P=$ NS). In multivariate logistic regression analysis, the IFN-related module scores improved the ability of the ELN category to predict therapeutic resistance, but not patient survival (Fig. 6AB; Table S7). Specifically, the myeloid inflammation score $(P=0.003)$, IFN- $\gamma$ signaling score $(P=0.014)$ and IFN downstream score $(P=0.034)$ significantly contributed to the model (Table S7). Gene sets defining gene modules 2 and 3 in Fig. 1, reflective of adaptive immune responses and BM infiltration with cells of the myeloid lineage, respectively, were not associated with either therapeutic resistance or patient survival.

We next tested the predictive and prognostic power of immune scores in silico using a broad collection of public transcriptomic data. We initially devised binary logistic regression models utilizing RNA-sequencing data from 196 patients on the Beat AML Master Trial with clinical response information (43). When considering disease type (primary versus secondary), WBC count and patient age at diagnosis, the inclusion of genes capturing IFN- $\gamma$-related biology significantly improved the predictive ability of the ELN risk category (AUROC $=0.921$ versus 0.709 with ELN cytogenetic risk alone; model $\chi^{2}=106.4$ versus 29.6 ; increased specificity $=4 \%$; increased sensitivity $=17 \%$; decreased false positive rate $=39 \%$; decreased false negative rate=18\%; Fig. 6C).

Confirming our findings in the PMCC and Beat AML cohorts, IFN-dominant, adaptive and myeloid mRNA profiles, when used in aggregate, stratified patients in the HOVON database (618 non-promyelocytic AML cases (44)) into subgroups with high and low immune infiltration (Fig. 6D-E). Individuals with immune-infiltrated AML had lower leukemia burden (median percentage of $\mathrm{BM}$ blasts $=56 \%$ versus $71 \%$ in patients with immune-depleted $\mathrm{AML} ; P<0.0001$ ) and tended to have more advanced age at diagnosis (median=51 years, range 15-74, versus 46 years, range $15-77 ; P=0.0067)$. A higher percentage of patients with IFN-dominant AML failed to achieve CR in response to induction chemotherapy when compared to non-IFNdominant AML cases (27.2\% versus $15.2 \% ; P=0.0004$, Fisher's exact test). In contrast, the 
occurrence of primary induction failure was not different when patients were dichotomized based on higher or lower than median adaptive module scores (21.4\% versus $21.0 \%$ IF rate; $P=$ NS) or myeloid module scores $(21.7 \%$ versus $20.7 \%$ IF rate; $P=$ NS). Gene set enrichment analysis (GSEA) with all transcripts in the HOVON dataset provided as input and ranked by the $\log _{2}$ fold-change between non-responders and responders confirmed the over-expression of curated hallmark gene sets linked to IFN- $\gamma$ responses and inflammatory responses in chemotherapy-refractory patients (Fig. 6F). When tested in a multinomial logistic regression model incorporating patient age, leukemia burden and ELN cytogenetic risk (available in 615 HOVON cases) (45), immune gene sets defining the IFN-dominant module significantly and independently predicted whether patients responded to induction chemotherapy and whether they experienced disease relapse (Table S8). In contrast, immune gene signatures were unable to assist the prediction of non-leukemic deaths (Table S8).

Mutations in tumor suppressor genes and transcription factors are enriched in immuneinfiltrated $A M L$ cases

It has recently been shown that genetic drivers of solid malignancies dictate neutrophil and Tcell recruitment, thus affecting the immune milieu of the tumor and assisting patient stratification (46). We asked whether clonal driver mutations may correlate with the immune subtypes that we identified herein. We therefore retrieved TCGA AML RNA-sequencing data from cBioPortal (http://www.cbioportal.org/) and computed immune cell type-specific and biological activity scores (22). IFN-related gene sets, including the TIS score, were higher in TCGA-AML cases with TP53 and RUNX1 mutations relative to molecular lesions that confer favorable or intermediate risk (Fig. S13A). In contrast, the majority of TCGA-AML cases with NPM1 mutations with or without FLT3-ITD (intermediate-risk and favorable-risk cases, respectively) were classified as immune-depleted. Clonal hematopoiesis of indeterminate potential (CHIP) is a hematological malignancy precursor condition defined by somatic mutations in leukemia-associated driver genes, including DNMT3A, TET2 and ASXL1, and associated with increased risk for inflammatory diseases of aging (47). Interestingly, the TIS 
score, but not the IFN- $\gamma$ score, was significantly higher in TCGA AML cases with CHIP-defining mutations compared with patient subgroups with other molecular lesions (Fig. S13B). When extending our in-silico analysis to the Beat AML cohort (281 cases in total), 16 out of 17 (94\%) TP53-mutated AMLs expressed higher amounts of genes implicated in downstream IFN signaling and higher CD8 transcripts and markers of cytotoxicity compared with TP53 wildtype cases (Fig. S14A-B).

IFN- $\gamma$-related gene expression and protein profiles correlate with anti-leukemia responses after flotetuzumab immunotherapy

Finally, we hypothesized that higher expression of IFN- $\gamma$-related genes in immune-infiltrated AML cases, while underpinning chemotherapy resistance, might identify patients with AML who derive benefit from immunotherapy with flotetuzumab (48), a CD3×CD123 dual affinity re-targeting (DART) molecule. BM samples collected prior to flotetuzumab treatment from 30 adult patients with chemotherapy-refractory or relapsed AML enrolled in the CP-MGD006-01 clinical trial (NCT\#02152956) were profiled using the PanCancer IO360 gene expression assay. Patients' characteristics are summarized in Table $\mathbf{S 9}$ and flotetuzumab anti-leukemic activity was defined as either $\mathrm{CR}, \mathrm{CR}$ with partial hematologic recovery (CRh), CR with incomplete hematologic recovery (CRi), partial response or overall benefit ( $>30 \%$ reduction in $\mathrm{BM}$ and/or blood blasts). BM samples from $92 \%$ of patients with evidence of flotetuzumab antileukemic activity (11 out of 12) had an immune-infiltrated TME relative to non-responders (Fig. 7A). The IFN-dominant module score was significantly higher in patients with chemotherapyrefractory $A M L$ compared with relapsed $A M L$ at time of flotetuzumab treatment, and in individuals with evidence of anti-leukemic activity compared to non-responders (Fig. 7B). Notably, the TIS score was a strong predictor of anti-leukemic responses to flotetuzumab, with an AUROC value of 0.847 (Fig. 7C). On-treatment BM samples (available in 19 patients at the end of cycle 1) displayed increased antigen presentation and immune activation relative to baseline samples, as reflected by higher TIS scores $(6.47 \pm 0.22$ versus $5.93 \pm 0.15, P=$ 
$0.0006)$, antigen processing machinery scores $(5.67 \pm 0.16$ versus $5.31 \pm 0.12, P=0.002)$, IFN$\gamma$ signaling scores $(3.58 \pm 0.27$ versus $2.81 \pm 0.24, P=0.0004)$ and $P D-L 1$ expression $(3.43 \pm 0.28$ versus 2.73 $\pm 0.21, P=0.0062$; Fig. 7D). GeoMx DSP of BM FFPE biopsies from a subgroup of 11 patients identified protein profiles at baseline that distinguish responders from nonresponders (Fig. 7E-F and Fig. S15). The IFN- $\gamma$-inducible molecule STING was upregulated post-cycle 1 of flotetuzumab in two patients who achieved CR (Fig. 7G). In these individuals, T-cell activation markers (CD27, CD45RO, CD44), Bcl-2, immune checkpoints (ICOS, PD-L2, CTLA4 and 4-1BB [CD137]) and CD4 were highly expressed in ROls with T-cell clustering (CD3 hotspots) around CD123 ${ }^{+}$AML blasts (Fig. 7H), supporting a local immune-modulatory effect of flotetuzumab. Overall, these data suggest a clinical benefit for AML patients with an immune-infiltrated TME and validate the translational relevance of our findings. 


\section{Discussion}

Using large cohorts of subjects, the current study reveals underlying transcriptomic features that stratify the TME of AML into immune subtypes and may assist therapeutic predictions by defining patients who will potentially derive the greatest benefit from immunotherapies. We identified two subtypes of differentially immune-infiltrated tumors, an observation that was validated in independent childhood and adult $A M L$ series, reinforcing the notion that unique molecular features can distinguish AML across age groups (35). The IFN-related gene sets identified in our study improved the prediction of therapeutic resistance following conventional ' $3+7$ ' cytarabine and anthracycline chemotherapy beyond that provided by the ELN cytogenetic risk category $(\mathrm{AUC}=0.815$ in PMCC cases [discovery series] and 0.870 in Beat AML cases [in silico validation series]) (49). In recent Southwestern Oncology Group (SWOG) and MD Anderson Cancer Center clinical trials, pretreatment covariates such as cytogenetic risk and age only yielded AUROCs of 0.65 and 0.59 for therapeutic resistance, respectively (41). Models that were established in the present study by incorporating IFN-related mRNA profiles also outperform a recently developed 29-gene and cytogenetic risk predictor of chemotherapy resistance $(A U R O C=0.76)(50)$. Our observation that CD8 exhaustion scores and PD1 scores are significantly higher in patients with ELN adverse risk compared with ELN favorable risk is congruent with a previous report showing unique transcriptional programs associated with ELN cytogenetic risk groups in $\mathrm{CD}^{+} \mathrm{T}$ cells from patients with $\mathrm{AML}(51)$. Intriguingly, an IFN-related DNA damage resistance signature (IRDS) correlates with resistance to adjuvant chemotherapy and with recurrence after radiotherapy in patients with breast cancer (42), suggesting that tumor cells over-expressing IRDS genes, including STAT1, ISG15 and IFIT1, as a result of chronic activation of the IFN signaling pathway might receive pro-survival rather than cytotoxic signals in response to DNA damage (52).

The immune-infiltrated AML cases were highly immune-suppressed, as indicated by elevated expression of IFN-inducible negative immune checkpoints and immunotherapy targets IDO1 and PD-L1, and simultaneously secreted high amounts of IFN- $\gamma$ and TNF- $\alpha$, a measure of polyfunctional Th1 responses (53). Furthermore, adults with relapsed AML in the SAL series 
expressed a higher magnitude of T-cell exhaustion molecules relative to matched pretreatment samples, suggesting the occurrence of escape from immune surveillance at the time of disease relapse (54). In general, solid tumors with a substantial T-cell component and displaying a type I immune response are associated with better OS and progression-free survival estimates (6). However, the highly proliferative IFN- $\gamma$-dominant solid tumors may correlate with a less favorable survival, despite being infiltrated with $C D 8^{+} T$ cells and harboring the highest type 1 macrophage signature scores (6). By utilizing targeted IGEP, our study highlights that the activation of IFN-related pathways and the relative abundance of immune cell types, including the over-expression of T-cell markers and TCR signaling intermediates in relapsed $A M L$ relative to disease onset, have negative prognostic implications in AML. This is conceivably the result of a non-productive anti-leukemia immune response and/or IFN-driven resistance to DNA damage induced by chemotherapeutic agents (20). Patients with immune-infiltrated AML at baseline had better OS estimates when treated with allogeneic HSCT compared with chemotherapy alone, suggesting that GVL effects were more profound in this patient subgroup. In contrast, no survival advantage from allogeneic HSCT was evident in patients with immune-depleted AML.

The heterogeneity of immune infiltration can also be determined by tumor cell-intrinsic factors, including chemokine secretion (55) and expression of cancer driver genes, all of which affect response to immunotherapies $(46,56,57)$. Interestingly, we detected associations between mutations in tumor suppressor and cancer driver genes and immune subtypes of AML and we identified TP53 mutations as being strongly correlated with an IFN- $\gamma$-dominant TME and with prognostic protein signatures, including the expression of PD-L1, FOXP3, PTEN and GZMB, that were revealed by spatially-resolved, highly multiplexed protein profiling. Notably, GZMB expression has recently been associated with features of exhaustion and senescence in AML $\mathrm{CD}^{+} \mathrm{T}$ cells (38). A recent study showed higher proportions of PD-L1-expressing CD8 ${ }^{+} \mathrm{T}$ cells, activation of IFN- $\gamma$-associated genes and favorable responses to pembrolizumab immunotherapy in TP53-mutated lung cancers (58). It is tempting to speculate that immune- 
infiltrated, TP53-mutated AML cases, which have very low response rates when treated with standard anthracycline-based and cytarabine-based induction chemotherapy, could benefit from $\mathrm{T}$ cell-targeting approaches and/or hypomethylating agents that potentially alter the immune surveillance of AML (59). PTEN loss has been correlated with reduced $\mathrm{CD}^{+} \mathrm{T}$-cell infiltration, with lower expression of IFNG and GZMB transcripts and with resistance to immune checkpoint blockade in metastatic melanoma and in primary sarcoma $(60,61)$. The higher expression of CD8 and GZMB that we observed in ROls with higher PTEN suggests that PTEN may represent a novel molecular driver of T-cell infiltration in AML. In addition, higher expression of GZMB correlated with worse clinical outcomes and individually separated AML patients into subgroups with different survival probabilities. In this respect, signatures of dysfunctional T cells, including high expression of GZMB and other transcripts associated with effector $\mathrm{CD}^{+}{ }^{+}$T-cell differentiation such as IFNG, may be increased in AML patients at diagnosis and persist with higher frequency only in chemotherapy non-responders (38). Finally, IFN- $\gamma$-related gene expression programs in the AML TME, including the TIS score, correlated with response to flotetuzumab immunotherapy in 30 heavily pre-treated patients with relapsed/refractory AML on clinical trial CP-MGD006-01. Flotetuzumab treatment was associated with increased expression of antigen processing machinery components, IFN- $\gamma$ signaling molecules such as STING, negative immune checkpoints and lymphocyte activation markers, including heightened $P D-L 1$ mRNA and protein, in BM ROls with CD3 hotspots. This finding provides a biological rationale for designing clinical studies with sequential flotetuzumab and immune checkpoint blockade in AML patients in remission with minimal residual disease.

The use of bulk BM aspirates is a potential limitation of our analysis. Future studies should use single-cell RNA sequencing of purified $\mathrm{CD}^{+} \mathrm{T}$ cells to further dissect individual variation in response to T-cell engagers (62). We also acknowledge that the detailed phenotypes, antigen specificities and intratumoral TCR repertoires of T cells in patients with immuneinfiltrated AML remain to be established (63). 
In conclusion, our work unveils the heterogeneity of the immune landscape of AML and provides a novel precision medicine-based conceptual framework for delivering $\mathrm{T}$ celltargeting immunotherapy to subgroups of patients with IFN- $\gamma$-dominant $A M L$, who may be refractory to conventional cytotoxic chemotherapy but responsive to T-cell engagers. The immunological stratification of pre-treatment BM samples may therefore enable rapid risk prediction and selection of frontline therapeutic modalities (11), in conjunction with cytogenetic and mutational information. 


\section{Materials and Methods}

\section{Study design}

The CP-MGD006-01 clinical trial (NCT\#02152956) is a multicenter, open-label, phase 1/2 dose escalation and dose expansion study. Thirty patients with primary refractory $(n=23)$ and relapsed $\operatorname{AML}(n=7)$ treated with flotetuzumab at the recommended phase 2 dose (500 $\mathrm{ng} / \mathrm{kg} /$ day) were included in the current analysis. Patients received a lead-in dose of flotetuzumab during week 1 , followed by $500 \mathrm{ng} / \mathrm{kg} /$ day during weeks $2-4$ of cycle 1 , and a 4day on/3-day off schedule for cycle 2 and beyond. Eligible patients were 18 years of age or older, with relapsed or refractory AML (according to WHO criteria) unlikely to benefit from cytotoxic chemotherapy defined as a) refractory to $\geq 2$ induction attempts (primary induction failure); b) first relapse with an initial complete remission (CR) duration $<6$ months (early relapse) or relapse in patients that achieve a CR lasting $\geq 6$ months following prior therapy (late relapse), or d) prior failure of hypomethylating agents. All participants were required to have Eastern Cooperative Oncology Group performance status of $\leq 2$, a peripheral blast count of $<20,000 / \mathrm{mm}^{3}$ at the time of first treatment, and adequate organ function. Patients with a prior history of allogeneic HSCT, active untreated autoimmune disorders, or active central nervous system leukemia were excluded. The trial was approved by the Institutional Review Boards of participating centers and was conducted according to the current International Conference on Harmonization (ICH) Guideline for Good Clinical Practice (ICH E6), and all applicable local and national regulations and ethical principles in accordance with the Helsinki Declaration. All participants provided written informed consent prior to enrollment.

BM aspirates were collected at baseline $(n=30)$ and after cycle 1 of flotetuzumab $(n=19)$ to evaluate the temporal immunological effects associated with therapeutic response. Disease status was assessed by modified IWG criteria. Anti-leukemic response was defined as either $\mathrm{CR}, \mathrm{CR}$ with incomplete hematologic recovery $(\mathrm{CRi}), \mathrm{CR}$ with partial hematologic recovery (CRh), partial remission (PR) or "other benefit" (OB; $>30 \%$ decrease in BM blasts). Nonresponders were individuals with either treatment failure (TF), stable disease (SD) or progressive disease (PD). Patient and disease characteristics are detailed in Table S8. 


\section{Patients' demographics (discovery cohorts)}

Patient and disease characteristics are detailed in Table 1. Primary patient specimens (nonpromyelocytic $\mathrm{AML}$ ) and associated clinical data were obtained on research protocols approved by the Investigational Review Boards of the Children's Hospital of Philadelphia (CHOP), USA and Princess Margaret Cancer Centre (PMCC), Canada and by the Ethics Committee of TU Dresden and Studienallianz Leukämie (SAL), Germany.

\section{RNA isolation and processing}

Messenger RNA was isolated and processed as previously described (45). For the PMCC, SAL and CHOP patient cohorts, 100-150 ng per sample of RNA extracted from 442 bulk BM aspirates from patients with AML treated with curative intent were analyzed on the NanoString nCounter FLEX analysis system using the PanCancer Immune [PCI] profiling panel (for research use only and not for use in diagnostic procedures), which measures mRNA expression of 770 genes representing 14 immune cell types, common checkpoint inhibitors, cancer testis antigens and genes covering both the innate and adaptive immune response without the need for amplification $(25,64,65)$. BM samples from patients receiving flotetuzumab immunotherapy were analyzed using the PanCancer IO 360 panel (for research use only and not for use in diagnostic procedures).

nCounter data quality control, data normalization and signature calculation

The reporter probe counts, i.e., the number of times the color-coded barcode for that gene is detected, were tabulated in a comma separated value (CSV) format for data analysis with the nSolver software package (version 4.0.62) and nSolver Advanced Analysis module (version 2.0.115; NanoString Technologies). The captured transcript counts were normalized to the geometric mean of the housekeeping reference genes included in the assay and the code set's internal positive controls. The relative abundance of immune cell types and immunooncology biological signatures were computed as previously published $(22,23)$. For samples 
run on the $\mathrm{PCI}$ profiling panel, we also calculated an approximation of the TIS using 16 of the 18 functional genes and 5 of the 10 housekeeper genes that are present in the PanCancer IO360 mRNA panel (18).

Gene ontology (GO) and gene set enrichment analysis (GSEA)

Metascape.org was used to enrich genes for GO biological processes and pathways. GSEA was performed using the GSEA software v.3.0 (Broad Institute, Cambridge, USA) (66). The hallmark IFN- $\gamma$ response (M5911) and inflammatory response gene sets (M5913) were downloaded from the Molecular Signature Database (MSigDB) $(22,23)$.

\section{GeoMx Digital Spatial Profiling (DSP)}

Ten FFPE BM biopsies from patients with newly diagnosed AML (SAL series) and 19 FFPE BM biopsies from patients receiving flotetuzumab immunotherapy $(n=11$ at baseline and $n=8$ post-cycle 1) were profiled using the prototype or commercial GeoMx DSP platform, respectively (Fig. S15). Samples were stained using 3 fluorescent visualization markers, CD3 (T cell), CD123 (myeloid blast), SYTO 83 or 13 (nuclei), and UV-cleavable oligo-labeled antibodies (panels are shown in Table S10). Stained slides were loaded on the DSP instrument and digitally scanned. Fluorescent scans were used to select 24 geometric regions of interest (ROIs) for molecular profiling $(27,67)$. The DSP instrument then UV-illuminated selected ROIs to release conjugated oligos and the micro-capillary fluidics system collected released oligos, which were counted on the nCounter system. Data were normalized to technical controls and area.

Intracellular cytokine staining

Cells were aliquoted into $12 \times 75 \mathrm{~mm}$ tubes $\left(0.5 \times 10^{6}\right.$ cells per tube $)$ in $500 \mu \mathrm{L}$ RPMI 1640 (Lonza) $+10 \%$ fetal bovine serum (FBS) (v/v). Two tubes were set up per sample, the first as an unstimulated control and the second stimulated with $50 \mathrm{ng} / \mathrm{mL}$ PMA and $1 \mu \mathrm{g} / \mathrm{mL}$ ionomycin (both from Sigma Aldrich). Samples also received $10 \mu \mathrm{g} / \mathrm{mL}$ brefeldin A (BioLegend), then were vortexed gently and incubated at $37^{\circ} \mathrm{C}$ for 5 hours. Following incubation, cells were 
washed in PBS then incubated in $100 \mu \mathrm{L}$ PBS containing $5 \mu \mathrm{L}$ Human FcR Blocking Reagent (Miltenyi Biotec), fluorescently labeled mAbs for surface markers of interest (CD3, CD4, CD8; BioLegend) and fixable LIVE/DEAD viability stain (Molecular Probes) for 30 minutes at $4^{\circ} \mathrm{C}$ protected from light. Unbound antibody was washed off using PBS, then cells were fixed by incubating in $200 \mu \mathrm{L} 1 \times$ True-Nuclear Fix Buffer (True-Nuclear Transcription Factor Buffer Set, BioLegend) for 20 minutes. Cells were washed in 1×Perm Buffer (True-Nuclear Transcription Factor Buffer Set, BioLegend) then resuspended in $100 \mu \mathrm{L}$ 1×Perm Buffer and fluorescentlylabeled mAbs for intracellular cytokines of interest (IL-2, IL-4, IL-10, IL-17, IFN- $\gamma$ and TNF- $\alpha$; antibody clones are provided in Table S11) and incubated for 20 minutes at room temperature protected from light. Unbound antibody was washed off using $1 \times$ Perm Buffer, cells were resuspended in $400 \mu \mathrm{L}$ PBS and immediately analyzed on a 3-laser, 10-color Gallios flow cytometer (Beckman Coulter).

\section{Data sources for in silico analyses}

The first data series (E-MTAB-3444), hereafter referred to as the HOVON series (44), was retrieved from Array Express and encompassed three independent cohorts of adults ( $\leq 60$ years) with de novo AML (last accessed on March 4, 2019). BM and blood samples were collected at diagnosis and were analyzed on the Affymetrix Human Genome U133 Plus 2.0 Microarray $(44,68)$. Patients were treated with curative intent according to the Dutch-Belgian Hematology-Oncology Cooperative Group and the Swiss Group for Clinical Cancer Research (HOVON/SAKK) AML-04, -04A, -29, $-32,-42,-42 \mathrm{~A},-43$ or -92 protocols (available at http://www.hovon.nl). Clinical annotations were provided by the authors. The second data series, hereafter referred to as The Cancer Genome Atlas (TCGA) series, consisted of RNAsequencing data (Illumina HiSeq2000) from 162 adult AML patients with complete cytogenetic, immunophenotypic and clinical annotation who were enrolled on Cancer and Leukemia Group B (CALGB) treatment protocols 8525, 8923, 9621, 9720, 10201 and 19808 (69). RNA and clinical data were retrieved from the TCGA data portal (https://tcgadata.nci.nih.gov/tcga/tcgaDownload.jsp). The third data series (Beat AML) was retrieved using 
the VIZOME user interface (http://www.vizome.org/aml/) and consisted of RNA-sequencing data from primary specimens from 281 AML patients with detailed clinical annotations, including diagnostic information, treatments, responses and outcomes treated on the Beat AML Master Trial (43). Patient and disease characteristics for in silico data sources are summarized in Data File S1.

\section{Statistical analyses}

Descriptive statistics included calculation of mean, median, SD, and proportions to summarize study outcomes. Comparisons were performed with the Mann-Whitney $U$ test for paired or unpaired data (two-sided), as appropriate, or with the ANOVA with correction for multiple comparisons. IBM SPSS Statistics (version 24) and GraphPad Prism (version 8) were used for statistical analyses. A two-sided $P$ value $<0.05$ was considered to reflect statistically significant differences. The log-rank (Mantel-Cox) test was used to compare survival distributions.

Therapeutic resistance was defined as failure to achieve complete remission (CR) despite not experiencing early treatment-related mortality (within 28 days of chemotherapy initiation; primary refractory cases) or as early relapse ( $<3$ months) after achieving CR (41). Overall survival (OS) was computed from the date of diagnosis to the date of death. Relapse-free survival (RFS) was measured from the date of first CR to the date of relapse or death. Subjects lost to follow-up were censored at their date of last known contact.

Binary logistic regression and multinomial logistic regression were used to ascertain the relative contribution of immune subtypes and other pretreatment covariates selected a priori based on known clinical relevance (ELN risk group, FLT3-ITD status, NPM1 mutational status, patient age at diagnosis and primary versus secondary $A M L)$ toward the predicted likelihood of response to induction chemotherapy, AML relapse and patient death (45). Competing risks regression analyses by the method of Fine and Gray were performed using STATA/IC (version 16.0) (32). Allogeneic HSCT, a potential confounder, was treated as an event whose occurrence precluded the occurrence of the primary clinical endpoint (death) (32). 


\section{Supplementary Materials}

Fig. S1. Immune gene signatures and survival in the AML discovery series (PMCC cohort; $\mathrm{n}=290$ cases).

Fig. S2: GeoMx digital spatial profiling (DSP) and region of interest (ROI) selection in a representative pre-treatment BM trephine biopsy (SAL series) with high T-cell infiltration.

Fig. S3: GeoMx digital spatial profiling (DSP) and region of interest (ROI) selection in a representative pre-treatment BM trephine biopsy (SAL series) with low T-cell infiltration.

Fig. S4: Highly multiplexed protein profiling in the SAL patient cohort.

Fig. S5: Correlation between CD3 infiltration and expression of immuno-oncology (IO)-related proteins as revealed by GeoMx digital spatial profiling (DSP) of BM trephine biopsies (SAL series).

Fig. S6: Expression of SIG3 genes in TCGA-AML cases and in healthy tissues.

Fig. S7: Association between immune gene signatures and patients' characteristics in the AML discovery series (PMCC cohort; $n=290$ cases).

Fig. S8: Clinical outcomes of patients with immune-infiltrated and immune-depleted AML treated with allogeneic HSCT (PMCC cohort).

Fig. S9: Immune gene signatures in the CHOP and SAL cohorts.

Fig. S10: Gene ontology and pathway analyses.

Fig. S11: Immune scores in AML patients (SAL cohort) at time of diagnosis and achievement of complete remission (paired samples).

Fig. S12: Intracellular cytokine staining of BM samples from patients with immune-infiltrated and immune-depleted AML.

Fig. S13: Immune subtypes associate with cancer driver gene mutations in The Cancer Genome Atlas (TCGA)-AML and Beat AML trial specimens.

Fig. S14: Immune signature scores and cancer driver gene mutations in The Cancer Genome Atlas (TCGA)-AML and Beat AML trial specimens.

Fig. S15: Selection of regions of interest in BM biopsies from patients receiving flotetuzumab immunotherapy. 
Table S1: Differentially expressed (DE) genes (false discovery rate<0.05) between ELN favorable-risk and adverse-risk AML (HOVON discovery cohort).

Table S2: Competing risks analysis for overall survival (OS) prediction (PMCC discovery cohort).

Table S3: Causes of death in patients receiving allogeneic hematopoietic stem cell transplantation (HSCT; PMCC cohort).

Table S4: Gene ontologies (GO) of and KEGG pathways captured by DE genes between adult (SAL cohort) and childhood AML (CHOP cohort).

Table S5: GO of and KEGG pathways captured by DE genes between adult AML patients at the time of diagnosis and achievement of complete remission (SAL cohort).

Table S6: GO of and KEGG pathways captured by DE genes between adult AML patients at the time of relapse and achievement of complete remission (SAL cohort).

Table S7: Binary logistic regression predicting therapeutic resistance (PMCC cohort).

Table S8: Multinomial logistic regression predicting therapeutic response (HOVON cohort).

Table S9: Study participants in the CP-MGD006-01 clinical trial (NCT\#02152956) of flotetuzumab immunotherapy.

Table S10: Protein panels used for GeoMx Digital Spatial Profiling (DSP) of FFPE bone marrow biopsies (SAL cohort $[A]$ and flotetuzumab cohort $[B]$ ).

Table S11: Antibody panel used for intracellular cytokine staining of bone marrow cell suspensions.

Data File S1: Patient and disease characteristics (in silico data sources). 


\section{References}

1. H. Dohner, D. J. Weisdorf, C. D. Bloomfield, Acute myeloid leukemia. N Engl J Med 373, 1136-1152 (2015).

2. E. Papaemmanuil, M. Gerstung, L. Bullinger, V. I. Gaidzik, P. Paschka, N. D. Roberts, N. E. Potter, M. Heuser, F. Thol, N. Bolli, G. Gundem, P. Van Loo, I. Martincorena, P. Ganly, L. Mudie, S. McLaren, S. O'Meara, K. Raine, D. R. Jones, J. W. Teague, A. P. Butler, M. F. Greaves, A. Ganser, K. Dohner, R. F. Schlenk, H. Dohner, P. J. Campbell, Genomic classification and prognosis in acute myeloid leukemia. N Engl J Med 374, 2209-2221 (2016).

3. A. Khwaja, M. Bjorkholm, R. E. Gale, R. L. Levine, C. T. Jordan, G. Ehninger, C. D. Bloomfield, E. Estey, A. Burnett, J. J. Cornelissen, D. A. Scheinberg, D. Bouscary, D. C. Linch, Acute myeloid leukaemia. Nat Rev Dis Primers 2, 16010 (2016).

4. C. C. Coombs, M. S. Tallman, R. L. Levine, Molecular therapy for acute myeloid leukaemia. Nat Rev Clin Oncol 13, 305-318 (2016).

5. M. Binnewies, E. W. Roberts, K. Kersten, V. Chan, D. F. Fearon, M. Merad, L. M. Coussens, D. I. Gabrilovich, S. Ostrand-Rosenberg, C. C. Hedrick, R. H. Vonderheide, M. J. Pittet, R. K. Jain, W. Zou, T. K. Howcroft, E. C. Woodhouse, R. A. Weinberg, M. F. Krummel, Understanding the tumor immune microenvironment (TIME) for effective therapy. Nat Med 24, 541-550 (2018).

6. V. Thorsson, D. L. Gibbs, S. D. Brown, D. Wolf, D. S. Bortone, T. H. Ou Yang, E. Porta-Pardo, G. F. Gao, C. L. Plaisier, J. A. Eddy, E. Ziv, A. C. Culhane, E. O. Paull, I. K. A. Sivakumar, A. J. Gentles, R. Malhotra, F. Farshidfar, A. Colaprico, J. S. Parker, L. E. Mose, N. S. Vo, J. Liu, Y. Liu, J. Rader, V. Dhankani, S. M. Reynolds, R. Bowlby, A. Califano, A. D. Cherniack, D. Anastassiou, D. Bedognetti, A. Rao, K. Chen, A. Krasnitz, H. Hu, T. M. Malta, H. Noushmehr, C. S. Pedamallu, S. Bullman, A. I. Ojesina, A. Lamb, W. Zhou, H. Shen, T. K. Choueiri, J. N. Weinstein, J. Guinney, J. Saltz, R. A. Holt, C. E. Rabkin, N. Cancer Genome Atlas Research, A. J. Lazar, J. S. Serody, E. G. Demicco, M. L. Disis, B. G. Vincent, L. Shmulevich, The immune landscape of cancer. Immunity 48, 812-830 (2018).

7. S. Gill, S. K. Tasian, M. Ruella, O. Shestova, Y. Li, D. L. Porter, M. Carroll, G. DanetDesnoyers, J. Scholler, S. A. Grupp, C. H. June, M. Kalos, Preclinical targeting of human acute myeloid leukemia and myeloablation using chimeric antigen receptormodified T cells. Blood 123, 2343-2354 (2014).

8. G. T. Gibney, L. M. Weiner, M. B. Atkins, Predictive biomarkers for checkpoint inhibitor-based immunotherapy. Lancet Oncol 17, e542-e551 (2016).

9. R. Cristescu, R. Mogg, M. Ayers, A. Albright, E. Murphy, J. Yearley, X. Sher, X. Q. Liu, H. Lu, M. Nebozhyn, C. Zhang, J. K. Lunceford, A. Joe, J. Cheng, A. L. Webber, N. Ibrahim, E. R. Plimack, P. A. Ott, T. Y. Seiwert, A. Ribas, T. K. McClanahan, J. E. Tomassini, A. Loboda, D. Kaufman, Pan-tumor genomic biomarkers for PD-1 checkpoint blockade-based immunotherapy. Science 362, (2018).

10. N. Daver, G. Garcia-Manero, S. Basu, P. C. Boddu, M. Alfayez, J. E. Cortes, M. Konopleva, F. Ravandi-Kashani, E. Jabbour, T. Kadia, G. M. Nogueras-Gonzalez, J. Ning, N. Pemmaraju, C. D. DiNardo, M. Andreeff, S. A. Pierce, T. Gordon, S. M. Kornblau, W. Flores, Z. Alhamal, C. Bueso-Ramos, J. L. Jorgensen, K. P. Patel, J. Blando, J. P. Allison, P. Sharma, H. Kantarjian, Efficacy, safety, and biomarkers of response to azacitidine and nivolumab in relapsed/refractory acute myeloid leukemia: A nonrandomized, open-label, phase II study. Cancer Discov 9, 370-383 (2019).

11. S. Rutella, S. E. Church, J. Vadakekolathu, E. Viboch, A. H. Sullivan, T. Hood, S. E. Warren, A. Cesano, R. La Motte-Mohs, J. Muth, H. Lelièvre, B. Lowenberg, J. F. DiPersio, J. K. Davidson-Moncada, Adaptive immune gene signatures correlate with response to flotetuzumab, a CD123 × CD3 bispecific DART® molecule, in patients with relapsed/refractory acute myeloid leukemia. Blood 132, 444-444 (2018).

12. D. S. Chen, I. Mellman, Elements of cancer immunity and the cancer-immune set point. Nature 541, 321-330 (2017). 
13. P. C. Tumeh, C. L. Harview, J. H. Yearley, I. P. Shintaku, E. J. Taylor, L. Robert, B. Chmielowski, M. Spasic, G. Henry, V. Ciobanu, A. N. West, M. Carmona, C. Kivork, E. Seja, G. Cherry, A. J. Gutierrez, T. R. Grogan, C. Mateus, G. Tomasic, J. A. Glaspy, R. O. Emerson, H. Robins, R. H. Pierce, D. A. Elashoff, C. Robert, A. Ribas, PD-1 blockade induces responses by inhibiting adaptive immune resistance. Nature 515, 568-571 (2014).

14. J. Zhao, A. X. Chen, R. D. Gartrell, A. M. Silverman, L. Aparicio, T. Chu, D. Bordbar, D. Shan, J. Samanamud, A. Mahajan, I. Filip, R. Orenbuch, M. Goetz, J. T. Yamaguchi, M. Cloney, C. Horbinski, R. V. Lukas, J. Raizer, A. I. Rae, J. Yuan, P. Canoll, J. N. Bruce, Y. M. Saenger, P. Sims, F. M. Iwamoto, A. M. Sonabend, R. Rabadan, Immune and genomic correlates of response to anti-PD-1 immunotherapy in glioblastoma. Nat Med 25, 462-469 (2019).

15. K. A. Schalper, M. E. Rodriguez-Ruiz, R. Diez-Valle, A. Lopez-Janeiro, A. Porciuncula, M. A. Idoate, S. Inoges, C. de Andrea, A. Lopez-Diaz de Cerio, S. Tejada, P. Berraondo, F. Villarroel-Espindola, J. Choi, A. Gurpide, M. Giraldez, I. Goicoechea, J. Gallego Perez-Larraya, M. F. Sanmamed, J. L. Perez-Gracia, I. Melero, Neoadjuvant nivolumab modifies the tumor immune microenvironment in resectable glioblastoma. Nat Med 25, 470-476 (2019).

16. T. F. Cloughesy, A. Y. Mochizuki, J. R. Orpilla, W. Hugo, A. H. Lee, T. B. Davidson, A. C. Wang, B. M. Ellingson, J. A. Rytlewski, C. M. Sanders, E. S. Kawaguchi, L. Du, G. Li, W. H. Yong, S. C. Gaffey, A. L. Cohen, I. K. Mellinghoff, E. Q. Lee, D. A. Reardon, B. J. O'Brien, N. A. Butowski, P. L. Nghiemphu, J. L. Clarke, I. C. ArrillagaRomany, H. Colman, T. J. Kaley, J. F. de Groot, L. M. Liau, P. Y. Wen, R. M. Prins, Neoadjuvant anti-PD-1 immunotherapy promotes a survival benefit with intratumoral and systemic immune responses in recurrent glioblastoma. Nat Med 25, 477-486 (2019).

17. J. M. Taube, R. A. Anders, G. D. Young, H. Xu, R. Sharma, T. L. McMiller, S. Chen, A. P. Klein, D. M. Pardoll, S. L. Topalian, L. Chen, Colocalization of inflammatory response with $\mathrm{B} 7-\mathrm{H} 1$ expression in human melanocytic lesions supports an adaptive resistance mechanism of immune escape. Sci Transl Med 4, 127ra137 (2012).

18. M. Ayers, J. Lunceford, M. Nebozhyn, E. Murphy, A. Loboda, D. R. Kaufman, A. Albright, J. D. Cheng, S. P. Kang, V. Shankaran, S. A. Piha-Paul, J. Yearley, T. Y. Seiwert, A. Ribas, T. K. McClanahan, IFN-gamma-related mRNA profile predicts clinical response to PD-1 blockade. J Clin Invest 127, 2930-2940 (2017).

19. P. A. Ott, Y. J. Bang, S. A. Piha-Paul, A. R. A. Razak, J. Bennouna, J. C. Soria, H. S. Rugo, R. B. Cohen, B. H. O'Neil, J. M. Mehnert, J. Lopez, T. Doi, E. M. J. van Brummelen, R. Cristescu, P. Yang, K. Emancipator, K. Stein, M. Ayers, A. K. Joe, J. K. Lunceford, T-cell-inflamed gene-expression profile, programmed death ligand 1 expression, and tumor mutational burden predict efficacy in patients treated with pembrolizumab across 20 cancers: KEYNOTE-028. J Clin Oncol 37, 318-327 (2019).

20. J. L. Benci, B. Xu, Y. Qiu, T. J. Wu, H. Dada, C. Twyman-Saint Victor, L. Cucolo, D. S. M. Lee, K. E. Pauken, A. C. Huang, T. C. Gangadhar, R. K. Amaravadi, L. M. Schuchter, M. D. Feldman, H. Ishwaran, R. H. Vonderheide, A. Maity, E. J. Wherry, A. J. Minn, Tumor interferon signaling regulates a multigenic resistance program to immune checkpoint blockade. Cell 167, 1540-1554 e1512 (2016).

21. S. W. Ng, A. Mitchell, J. A. Kennedy, W. C. Chen, J. McLeod, N. Ibrahimova, A. Arruda, A. Popescu, V. Gupta, A. D. Schimmer, A. C. Schuh, K. W. Yee, L. Bullinger, T. Herold, D. Gorlich, T. Buchner, W. Hiddemann, W. E. Berdel, B. Wormann, M. Cheok, C. Preudhomme, H. Dombret, K. Metzeler, C. Buske, B. Lowenberg, P. J. Valk, P. W. Zandstra, M. D. Minden, J. E. Dick, J. C. Wang, A 17-gene stemness score for rapid determination of risk in acute leukaemia. Nature 540, 433-437 (2016).

22. P. Danaher, S. Warren, A. Cesano, Development of gene expression signatures characterizing the tumor-immune interaction. J Clin Oncol 36, 205-205 (2018). 
23. P. Danaher, S. Warren, L. Dennis, L. D'Amico, A. White, M. L. Disis, M. A. Geller, K. Odunsi, J. Beechem, S. P. Fling, Gene expression markers of Tumor Infiltrating Leukocytes. J Immunother Cancer 5, 18 (2017).

24. K. Mrozek, G. Marcucci, D. Nicolet, K. S. Maharry, H. Becker, S. P. Whitman, K. H. Metzeler, S. Schwind, Y. Z. Wu, J. Kohlschmidt, M. J. Pettenati, N. A. Heerema, A. W. Block, S. R. Patil, M. R. Baer, J. E. Kolitz, J. O. Moore, A. J. Carroll, R. M. Stone, R. A. Larson, C. D. Bloomfield, Prognostic significance of the European LeukemiaNet standardized system for reporting cytogenetic and molecular alterations in adults with acute myeloid leukemia. J Clin Oncol 30, 4515-4523 (2012).

25. J. Vadakekolathu, T. Patel, S. Reeder, H. Schaarschmidt, M. Schmitz, M.

Bornhäuser, S. E. Warren, T. Hood, P. Danaher, A. Cesano, J. M. Beechem, A. G. Pockley, S. K. Tasian, S. Rutella, Immune gene expression profiling in children and adults with acute myeloid leukemia identifies distinct phenotypic patterns. Blood 130, 3942-3942 (2017).

26. S. Spranger, R. M. Spaapen, Y. Zha, J. Williams, Y. Meng, T. T. Ha, T. F. Gajewski, Up-regulation of PD-L1, IDO, and Tregs in the melanoma tumor microenvironment is driven by CD8+ T cells. Sci Transl Med 5, 200 ra116 (2013).

27. C. U. Blank, E. A. Rozeman, L. F. Fanchi, K. Sikorska, B. van de Wiel, P. Kvistborg, O. Krijgsman, M. van den Braber, D. Philips, A. Broeks, J. V. van Thienen, H. A. Mallo, S. Adriaansz, S. Ter Meulen, L. M. Pronk, L. G. Grijpink-Ongering, A. Bruining, R. M. Gittelman, S. Warren, H. van Tinteren, D. S. Peeper, J. Haanen, A. C. J. van Akkooi, T. N. Schumacher, Neoadjuvant versus adjuvant ipilimumab plus nivolumab in macroscopic stage III melanoma. Nat Med 24, 1655-1661 (2018).

28. R. M. Zemek, E. De Jong, W. L. Chin, I. S. Schuster, V. S. Fear, T. H. Casey, C. Forbes, S. J. Dart, C. Leslie, A. Zaitouny, M. Small, L. Boon, A. R. R. Forrest, D. O. Muiri, M. A. Degli-Esposti, M. J. Millward, A. K. Nowak, T. Lassmann, A. Bosco, R. A. Lake, W. J. Lesterhuis, Sensitization to immune checkpoint blockade through activation of a STAT1/NK axis in the tumor microenvironment. Sci Transl Med 11, (2019).

29. A. Ehninger, M. Kramer, C. Rollig, C. Thiede, M. Bornhauser, M. von Bonin, M. Wermke, A. Feldmann, M. Bachmann, G. Ehninger, U. Oelschlagel, Distribution and levels of cell surface expression of CD33 and CD123 in acute myeloid leukemia. Blood Cancer J 4, e218 (2014).

30. G. Ciriello, E. Cerami, C. Sander, N. Schultz, Mutual exclusivity analysis identifies oncogenic network modules. Genome Res 22, 398-406 (2012).

31. J. Versluis, F. E. In 't Hout, R. Devillier, W. L. van Putten, M. G. Manz, M. C. Vekemans, M. C. Legdeur, J. R. Passweg, J. Maertens, J. Kuball, B. J. Biemond, P. J. Valk, B. A. van der Reijden, G. Meloni, H. C. Schouten, E. Vellenga, T. Pabst, R. Willemze, B. Lowenberg, G. Ossenkoppele, F. Baron, G. Huls, J. J. Cornelissen, Comparative value of post-remission treatment in cytogenetically normal AML subclassified by NPM1 and FLT3-ITD allelic ratio. Leukemia 31, 26-33 (2017).

32. S. lacobelli, E. S. Committee, Suggestions on the use of statistical methodologies in studies of the European Group for Blood and Marrow Transplantation. Bone Marrow Transplant 48 Suppl 1, S1-37 (2013).

33. B. C. Medeiros, Interpretation of clinical endpoints in trials of acute myeloid leukemia. Leuk Res 68, 32-39 (2018).

34. J. E. Farrar, H. L. Schuback, R. E. Ries, D. Wai, O. A. Hampton, L. R. Trevino, T. A. Alonzo, J. M. Guidry Auvil, T. M. Davidsen, P. Gesuwan, L. Hermida, D. M. Muzny, N. Dewal, N. Rustagi, L. R. Lewis, A. S. Gamis, D. A. Wheeler, M. A. Smith, D. S. Gerhard, S. Meshinchi, Genomic profiling of pediatric acute myeloid leukemia reveals a changing mutational landscape from disease diagnosis to relapse. Cancer Res 76, 2197-2205 (2016).

35. H. Bolouri, J. E. Farrar, T. Triche, Jr., R. E. Ries, E. L. Lim, T. A. Alonzo, Y. Ma, R. Moore, A. J. Mungall, M. A. Marra, J. Zhang, X. Ma, Y. Liu, Y. Liu, J. M. G. Auvil, T. M. Davidsen, P. Gesuwan, L. C. Hermida, B. Salhia, S. Capone, G. Ramsingh, C. M. 
Zwaan, S. Noort, S. R. Piccolo, E. A. Kolb, A. S. Gamis, M. A. Smith, D. S. Gerhard, $S$. Meshinchi, The molecular landscape of pediatric acute myeloid leukemia reveals recurrent structural alterations and age-specific mutational interactions. Nat Med 24, 103-112 (2018).

36. R. G. Majzner, S. Heitzeneder, C. L. Mackall, Harnessing the immunotherapy revolution for the treatment of childhood cancers. Cancer Cell 31, 476-485 (2017).

37. S. S. Chung, W. S. Eng, W. Hu, M. Khalaj, F. E. Garrett-Bakelman, M. Tavakkoli, R. L. Levine, M. Carroll, V. M. Klimek, A. M. Melnick, C. Y. Park, CD99 is a therapeutic target on disease stem cells in myeloid malignancies. Sci Trans/ Med 9, (2017).

38. H. A. Knaus, S. Berglund, H. Hackl, A. L. Blackford, J. F. Zeidner, R. MontielEsparza, R. Mukhopadhyay, K. Vanura, B. R. Blazar, J. E. Karp, L. Luznik, I. Gojo, Signatures of CD8+ T cell dysfunction in AML patients and their reversibility with response to chemotherapy. JCl Insight 3, e120974 (2018).

39. D. V. Dolfi, K. D. Mansfield, A. M. Polley, S. A. Doyle, G. J. Freeman, H. Pircher, K. E. Schmader, E. J. Wherry, Increased T-bet is associated with senescence of influenza virus-specific CD8 T cells in aged humans. J Leukoc Bio/ 93, 825-836 (2013).

40. M. A. Paley, D. C. Kroy, P. M. Odorizzi, J. B. Johnnidis, D. V. Dolfi, B. E. Barnett, E. K. Bikoff, E. J. Robertson, G. M. Lauer, S. L. Reiner, E. J. Wherry, Progenitor and terminal subsets of CD8+ T cells cooperate to contain chronic viral infection. Science 338, 1220-1225 (2012).

41. R. B. Walter, M. Othus, G. Borthakur, F. Ravandi, J. E. Cortes, S. A. Pierce, F. R. Appelbaum, H. A. Kantarjian, E. H. Estey, Prediction of early death after induction therapy for newly diagnosed acute myeloid leukemia with pretreatment risk scores: a novel paradigm for treatment assignment. J Clin Oncol 29, 4417-4423 (2011).

42. R. R. Weichselbaum, H. Ishwaran, T. Yoon, D. S. Nuyten, S. W. Baker, N. Khodarev, A. W. Su, A. Y. Shaikh, P. Roach, B. Kreike, B. Roizman, J. Bergh, Y. Pawitan, M. J. van de Vijver, A. J. Minn, An interferon-related gene signature for DNA damage resistance is a predictive marker for chemotherapy and radiation for breast cancer. Proc Natl Acad Sci U S A 105, 18490-18495 (2008).

43. J. W. Tyner, C. E. Tognon, D. Bottomly, B. Wilmot, S. E. Kurtz, S. L. Savage, N. Long, A. R. Schultz, E. Traer, M. Abel, A. Agarwal, A. Blucher, U. Borate, J. Bryant, R. Burke, A. Carlos, R. Carpenter, J. Carroll, B. H. Chang, C. Coblentz, A. d'Almeida, R. Cook, A. Danilov, K. T. Dao, M. Degnin, D. Devine, J. Dibb, D. K. t. Edwards, C. A. Eide, I. English, J. Glover, R. Henson, H. Ho, A. Jemal, K. Johnson, R. Johnson, B. Junio, A. Kaempf, J. Leonard, C. Lin, S. Q. Liu, P. Lo, M. M. Loriaux, S. Luty, T. Macey, J. MacManiman, J. Martinez, M. Mori, D. Nelson, C. Nichols, J. Peters, J. Ramsdill, A. Rofelty, R. Schuff, R. Searles, E. Segerdell, R. L. Smith, S. E. Spurgeon, T. Sweeney, A. Thapa, C. Visser, J. Wagner, K. Watanabe-Smith, K. Werth, J. Wolf, L. White, A. Yates, H. Zhang, C. R. Cogle, R. H. Collins, D. C. Connolly, M. W. Deininger, L. Drusbosky, C. S. Hourigan, C. T. Jordan, P. Kropf, T. L. Lin, M. E. Martinez, B. C. Medeiros, R. R. Pallapati, D. A. Pollyea, R. T. Swords, J. M. Watts, S. J. Weir, D. L. Wiest, R. M. Winters, S. K. McWeeney, B. J. Druker, Functional genomic landscape of acute myeloid leukaemia. Nature 562, 526-531 (2018).

44. P. J. Valk, R. G. Verhaak, M. A. Beijen, C. A. Erpelinck, S. Barjesteh van Waalwijk van Doorn-Khosrovani, J. M. Boer, H. B. Beverloo, M. J. Moorhouse, P. J. van der Spek, B. Lowenberg, R. Delwel, Prognostically useful gene-expression profiles in acute myeloid leukemia. N Engl J Med 350, 1617-1628 (2004).

45. S. Wagner, J. Vadakekolathu, S. K. Tasian, H. Altmann, M. Bornhauser, A. G. Pockley, G. R. Ball, S. Rutella, A parsimonious 3-gene signature predicts clinical outcomes in an acute myeloid leukemia multicohort study. Blood Adv 3, 1330-1346 (2019).

46. M. Bezzi, N. Seitzer, T. Ishikawa, M. Reschke, M. Chen, G. Wang, C. Mitchell, C. Ng, J. Katon, A. Lunardi, S. Signoretti, J. G. Clohessy, J. Zhang, P. P. Pandolfi, Diverse 
genetic-driven immune landscapes dictate tumor progression through distinct mechanisms. Nat Med 24, 165-175 (2018).

47. E. K. Cook, T. Izukawa, S. Young, G. Rosen, M. Jamali, L. Zhang, D. Johnson, E. Bain, J. Hilland, C. K. Ferrone, J. Buckstein, J. Francis, B. Momtaz, A. J. M. McNaughton, X. Liu, B. Snetsinger, R. Buckstein, M. J. Rauh, Comorbid and inflammatory characteristics of genetic subtypes of clonal hematopoiesis. Blood Adv 3, 2482-2486 (2019).

48. G. R. Chichili, L. Huang, H. Li, S. Burke, L. He, Q. Tang, L. Jin, S. Gorlatov, V. Ciccarone, F. Chen, S. Koenig, M. Shannon, R. Alderson, P. A. Moore, S. Johnson, E. Bonvini, A CD3xCD123 bispecific DART for redirecting host T cells to myelogenous leukemia: preclinical activity and safety in nonhuman primates. Sci Transl Med 7, 289ra282 (2015).

49. R. B. Walter, M. Othus, A. K. Burnett, B. Lowenberg, H. M. Kantarjian, G. J. Ossenkoppele, R. K. Hills, F. Ravandi, T. Pabst, A. Evans, S. R. Pierce, M. C. Vekemans, F. R. Appelbaum, E. H. Estey, Resistance prediction in AML: analysis of 4601 patients from MRC/NCRI, HOVON/SAKK, SWOG and MD Anderson Cancer Center. Leukemia 29, 312-320 (2015).

50. T. Herold, V. Jurinovic, A. M. N. Batcha, S. A. Bamopoulos, M. Rothenberg-Thurley, B. Ksienzyk, L. Hartmann, P. A. Greif, J. Phillippou-Massier, S. Krebs, H. Blum, S. Amler, S. Schneider, N. Konstandin, M. C. Sauerland, D. Gorlich, W. E. Berdel, B. J. Wormann, J. Tischer, M. Subklewe, S. K. Bohlander, J. Braess, W. Hiddemann, K. H. Metzeler, U. Mansmann, K. Spiekermann, A 29-gene and cytogenetic score for the prediction of resistance to induction treatment in acute myeloid leukemia. Haematologica 103, 456-465 (2018).

51. R. Radpour, C. Riether, C. Simillion, S. Hopner, R. Bruggmann, A. F. Ochsenbein, CD8+ T cells expand stem and progenitor cells in favorable but not adverse risk acute myeloid leukemia. Leukemia 33, 2379-2392 (2019).

52. N. N. Khodarev, M. Beckett, E. Labay, T. Darga, B. Roizman, R. R. Weichselbaum, STAT1 is overexpressed in tumors selected for radioresistance and confers protection from radiation in transduced sensitive cells. Proc Natl Acad Sci U S A 101, 1714-1719 (2004).

53. Q. Han, N. Bagheri, E. M. Bradshaw, D. A. Hafler, D. A. Lauffenburger, J. C. Love, Polyfunctional responses by human $T$ cells result from sequential release of cytokines. Proc Natl Acad Sci U S A 109, 1607-1612 (2012).

54. M. J. Christopher, A. A. Petti, M. P. Rettig, C. A. Miller, E. Chendamarai, E. J. Duncavage, J. M. Klco, N. M. Helton, M. O'Laughlin, C. C. Fronick, R. S. Fulton, R. K. Wilson, L. D. Wartman, J. S. Welch, S. E. Heath, J. D. Baty, J. E. Payton, T. A. Graubert, D. C. Link, M. J. Walter, P. Westervelt, T. J. Ley, J. F. DiPersio, Immune Escape of Relapsed AML Cells after Allogeneic Transplantation. N Engl J Med 379, 2330-2341 (2018).

55. J. Li, K. T. Byrne, F. Yan, T. Yamazoe, Z. Chen, T. Baslan, L. P. Richman, J. H. Lin, Y. H. Sun, A. J. Rech, D. Balli, C. A. Hay, Y. Sela, A. J. Merrell, S. M. Liudahl, N. Gordon, R. J. Norgard, S. Yuan, S. Yu, T. Chao, S. Ye, T. S. K. Eisinger-Mathason, R. B. Faryabi, J. W. Tobias, S. W. Lowe, L. M. Coussens, E. J. Wherry, R. H. Vonderheide, B. Z. Stanger, Tumor cell-intrinsic factors underlie heterogeneity of immune cell infiltration and response to immunotherapy. Immunity 49, 178-193 e177 (2018).

56. J. J. Luke, R. Bao, R. F. Sweis, S. Spranger, T. F. Gajewski, WNT/beta-catenin pathway activation correlates with immune exclusion across human cancers. Clin Cancer Res, (2019).

57. Y. Senbabaoglu, R. S. Gejman, A. G. Winer, M. Liu, E. M. Van Allen, G. de Velasco, D. Miao, I. Ostrovnaya, E. Drill, A. Luna, N. Weinhold, W. Lee, B. J. Manley, D. N. Khalii, S. D. Kaffenberger, Y. Chen, L. Danilova, M. H. Voss, J. A. Coleman, P. Russo, V. E. Reuter, T. A. Chan, E. H. Cheng, D. A. Scheinberg, M. O. Li, T. K. Choueiri, J. J. Hsieh, C. Sander, A. A. Hakimi, Tumor immune microenvironment 
characterization in clear cell renal cell carcinoma identifies prognostic and immunotherapeutically relevant messenger RNA signatures. Genome Biol 17, 231 (2016).

58. Z. Y. Dong, W. Z. Zhong, X. C. Zhang, J. Su, Z. Xie, S. Y. Liu, H. Y. Tu, H. J. Chen, Y. L. Sun, Q. Zhou, J. J. Yang, X. N. Yang, J. X. Lin, H. H. Yan, H. R. Zhai, L. X. Yan, R. Q. Liao, S. P. Wu, Y. L. Wu, Potential predictive value of TP53 and KRAS mutation status for response to PD-1 blockade immunotherapy in lung adenocarcinoma. Clin Cancer Res 23, 3012-3024 (2017).

59. J. S. Welch, A. A. Petti, C. A. Miller, C. C. Fronick, M. O'Laughlin, R. S. Fulton, R. K. Wilson, J. D. Baty, E. J. Duncavage, B. Tandon, Y. S. Lee, L. D. Wartman, G. L. Uy, A. Ghobadi, M. H. Tomasson, I. Pusic, R. Romee, T. A. Fehniger, K. E. StockerlGoldstein, R. Vij, S. T. Oh, C. N. Abboud, A. F. Cashen, M. A. Schroeder, M. A. Jacoby, S. E. Heath, K. Luber, M. R. Janke, A. Hantel, N. Khan, M. J. Sukhanova, R. W. Knoebel, W. Stock, T. A. Graubert, M. J. Walter, P. Westervelt, D. C. Link, J. F. DiPersio, T. J. Ley, TP53 and decitabine in acute myeloid leukemia and myelodysplastic syndromes. N Engl J Med 375, 2023-2036 (2016).

60. W. Peng, J. Q. Chen, C. Liu, S. Malu, C. Creasy, M. T. Tetzlaff, C. Xu, J. A. McKenzie, C. Zhang, X. Liang, L. J. Williams, W. Deng, G. Chen, R. Mbofung, A. J. Lazar, C. A. Torres-Cabala, Z. A. Cooper, P. L. Chen, T. N. Tieu, S. Spranger, X. Yu, C. Bernatchez, M. A. Forget, C. Haymaker, R. Amaria, J. L. McQuade, I. C. Glitza, T. Cascone, H. S. Li, L. N. Kwong, T. P. Heffernan, J. Hu, R. L. Bassett, Jr., M. W. Bosenberg, S. E. Woodman, W. W. Overwijk, G. Lizee, J. Roszik, T. F. Gajewski, J. A. Wargo, J. E. Gershenwald, L. Radvanyi, M. A. Davies, P. Hwu, Loss of PTEN promotes resistance to T cell-mediated immunotherapy. Cancer Discov 6, 202-216 (2016).

61. S. George, D. Miao, G. D. Demetri, D. Adeegbe, S. J. Rodig, S. Shukla, M. Lipschitz, A. Amin-Mansour, C. P. Raut, S. L. Carter, P. Hammerman, G. J. Freeman, C. J. Wu, P. A. Ott, K. K. Wong, E. M. Van Allen, Loss of PTEN Is associated with resistance to anti-PD-1 checkpoint blockade therapy in metastatic uterine leiomyosarcoma. Immunity 46, 197-204 (2017).

62. B. P. Fairfax, C. A. Taylor, R. A. Watson, I. Nassiri, S. Danielli, H. Fang, E. A. Mahe, R. Cooper, V. Woodcock, Z. Traill, M. H. Al-Mossawi, J. C. Knight, P. Klenerman, M. Payne, M. R. Middleton, Peripheral CD8(+) T cell characteristics associated with durable responses to immune checkpoint blockade in patients with metastatic melanoma. Nat Med 26, 193-199 (2020).

63. T. D. Wu, S. Madireddi, P. E. de Almeida, R. Banchereau, Y. J. Chen, A. S. Chitre, E. Y. Chiang, H. Iftikhar, W. E. O'Gorman, A. Au-Yeung, C. Takahashi, L. D. Goldstein, C. Poon, S. Keerthivasan, D. E. de Almeida Nagata, X. Du, H. M. Lee, K. L. Banta, S. Mariathasan, M. Das Thakur, M. A. Huseni, M. Ballinger, I. Estay, P. Caplazi, Z. Modrusan, L. Delamarre, I. Mellman, R. Bourgon, J. L. Grogan, Peripheral T cell expansion predicts tumour infiltration and clinical response. Nature, (2020).

64. T. Sugio, K. Miyawaki, K. Kato, K. Sasaki, K. Yamada, J. Iqbal, T. Miyamoto, K. Ohshima, T. Maeda, H. Miyoshi, K. Akashi, Microenvironmental immune cell signatures dictate clinical outcomes for PTCL-NOS. Blood Adv 2, 2242-2252 (2018).

65. J. E. Payton, N. R. Grieselhuber, L. W. Chang, M. Murakami, G. K. Geiss, D. C. Link, R. Nagarajan, M. A. Watson, T. J. Ley, High throughput digital quantification of mRNA abundance in primary human acute myeloid leukemia samples. $J$ Clin Invest 119, 1714-1726 (2009).

66. A. Subramanian, P. Tamayo, V. K. Mootha, S. Mukherjee, B. L. Ebert, M. A. Gillette, A. Paulovich, S. L. Pomeroy, T. R. Golub, E. S. Lander, J. P. Mesirov, Gene set enrichment analysis: a knowledge-based approach for interpreting genome-wide expression profiles. Proc Natl Acad Sci U S A 102, 15545-15550 (2005).

67. S. Rutella, J. Vadakekolathu, H. Altmann, T. Patel, S. Reeder, Y. Liang, M. Schmitz, T. Hood, P. Danaher, S. Warren, A. Cesano, J. M. Beechem, A. G. Pockley, S. K. 
Tasian, M. Bornhäuser, Capturing the complexity of the immune microenvironment of acute myeloid leukemia with 3D biology technology. J Clin Oncol 36, 50-50 (2018).

68. V. Stavropoulou, S. Kaspar, L. Brault, M. A. Sanders, S. Juge, S. Morettini, A. Tzankov, M. Iacovino, I. J. Lau, T. A. Milne, H. Royo, M. Kyba, P. J. M. Valk, A. Peters, J. Schwaller, MLL-AF9 expression in hematopoietic stem cells drives a highly invasive AML expressing EMT-related genes linked to poor outcome. Cancer Cell $\mathbf{3 0}$, 43-58 (2016).

69. T. J. Ley, C. Miller, L. Ding, B. J. Raphael, A. J. Mungall, A. Robertson, K. Hoadley, T. J. Triche, Jr., P. W. Laird, J. D. Baty, L. L. Fulton, R. Fulton, S. E. Heath, J. Kalicki-Veizer, C. Kandoth, J. M. Klco, D. C. Koboldt, K. L. Kanchi, S. Kulkarni, T. L. Lamprecht, D. E. Larson, L. Lin, C. Lu, M. D. McLellan, J. F. McMichael, J. Payton, H. Schmidt, D. H. Spencer, M. H. Tomasson, J. W. Wallis, L. D. Wartman, M. A. Watson, J. Welch, M. C. Wendl, A. Ally, M. Balasundaram, I. Birol, Y. Butterfield, R. Chiu, A. Chu, E. Chuah, H. J. Chun, R. Corbett, N. Dhalla, R. Guin, A. He, C. Hirst, M. Hirst, R. A. Holt, S. Jones, A. Karsan, D. Lee, H. I. Li, M. A. Marra, M. Mayo, R. A. Moore, K. Mungall, J. Parker, E. Pleasance, P. Plettner, J. Schein, D. Stoll, L.

Swanson, A. Tam, N. Thiessen, R. Varhol, N. Wye, Y. Zhao, S. Gabriel, G. Getz, C. Sougnez, L. Zou, M. D. Leiserson, F. Vandin, H. T. Wu, F. Applebaum, S. B. Baylin, R. Akbani, B. M. Broom, K. Chen, T. C. Motter, K. Nguyen, J. N. Weinstein, N. Zhang, M. L. Ferguson, C. Adams, A. Black, J. Bowen, J. Gastier-Foster, T. Grossman, T. Lichtenberg, L. Wise, T. Davidsen, J. A. Demchok, K. R. Shaw, M. Sheth, H. J. Sofia, L. Yang, J. R. Downing, G. Eley, Genomic and epigenomic landscapes of adult de novo acute myeloid leukemia. N Engl J Med 368, 2059-2074 (2013).

70. R. Edgar, M. Domrachev, A. E. Lash, Gene Expression Omnibus: NCBI gene expression and hybridization array data repository. Nucleic Acids Res 30, 207-210 (2002).

71. T. Metsalu, J. Vilo, ClustVis: a web tool for visualizing clustering of multivariate data using Principal Component Analysis and heatmap. Nucleic Acids Res 43, W566-570 (2015).

72. Z. Tang, B. Kang, C. Li, T. Chen, Z. Zhang, GEPIA2: an enhanced web server for large-scale expression profiling and interactive analysis. Nucleic Acids Res 47, W556-W560 (2019).

73. M. Lauten, A. Moricke, R. Beier, M. Zimmermann, M. Stanulla, B. Meissner, E. Odenwald, A. Attarbaschi, C. Niemeyer, F. Niggli, H. Riehm, M. Schrappe, Prediction of outcome by early bone marrow response in childhood acute lymphoblastic leukemia treated in the ALL-BFM 95 trial: differential effects in precursor B-cell and T-cell leukemia. Haematologica 97, 1048-1056 (2012).

74. W. Kern, T. Haferlach, C. Schoch, H. Loffler, W. Gassmann, A. Heinecke, M. C. Sauerland, W. Berdel, T. Buchner, W. Hiddemann, Early blast clearance by remission induction therapy is a major independent prognostic factor for both achievement of complete remission and long-term outcome in acute myeloid leukemia: data from the German AML Cooperative Group (AMLCG) 1992 Trial. Blood 101, 64-70 (2003).

\section{Acknowledgments}

\section{Funding}

This work was supported by grants from the Qatar National Research Fund (NPRP8-2297-3494 to SR); the Roger Counter Foundation, United Kingdom (to AGP and SR); the John and Lucille van Geest Foundation (to AGP and SR); the James Skillington Challenge for Leukemia 
(to AGP); the NCl K08 CA184418 and 1 U01 CA232486 and the Andrew McDonough B+ Foundation (to SKT); and R35CA210084 (to JFD). National Cancer Institute of the National Institutes of Health under Award Number R50CA211466 (MPR). St Baldrick's Foundation Stand Up To Cancer (SU2C) Pediatric Cancer Dream Team Translational Research Grant (SU2C-AACR-DT-27-17 to SKT). Stand Up To Cancer is a division of the Entertainment Industry Foundation. Research grants are administered by the American Association for Cancer Research, the scientific partner of SU2C. The Study Alliance of Leukemia (www.salaml.org) is gratefully acknowledged for providing primary patient material and clinical data.

\section{Author contributions}

Concept and design: S. Rutella

Development of methodology: J. Vadakekolathu, T. Hood, S.E. Church, S. Reeder, S.E. Warren, Y. Liang, T.H. Smith, M.D. Bailey, J. Gowen-MacDonald, S. Rutella Acquired, consented and managed patients; processed patient samples: M.D. Minden, H. Altmann, T. Patel, N. Ibrahimova, A. Arruda, J. Muth, P.J.M. Valk, B. Löwenberg, M. Bornhäuser, S.K. Tasian, M.P. Rettig, J.F. DiPersio

Analysis and interpretation of data: J. Vadakekolathu, M.D. Minden, T. Hood, S.E. Church, S. Reeder, A.H. Sullivan, E.J. Viboch, S.E. Warren, Y. Liang, T.H. Smith, G.A. Foulds, M.D. Bailey, J. Gowen-MacDonald, M. Schmitz, A. Cesano, P.J.M. Valk, B. Löwenberg, A.G. Pockley, M. Bornhäuser, S.K. Tasian, J. Davidson-Moncada, J.F. DiPersio, S. Rutella Clinical trial implementation: J.F. DiPersio was principal investigator at Washington University in St. Louis, St. Louis, United States of America. B. Löwenberg was principal investigator at Erasmus University Medical Centre, Rotterdam, Netherlands.

Writing of the manuscript: S. Rutella

Review and/or revision of the manuscript: J. Vadakekolathu, M.D. Minden, T. Hood, S.E. Church, E.J. Viboch, S.E. Warren, G.A. Foulds, M. Schmitz, A. Cesano, P.J.M. Valk, B. Löwenberg, A.G. Pockley, M. Bornhäuser, S.K. Tasian, M.P. Rettig, J. Davidson-Moncada, J.F. DiPersio, S. Rutella 
Study supervision: S. Rutella

Competing interests

John Muth, Jan Davidson-Moncada: Employees, MacroGenics Inc., Rockville, MD, USA;

Sarah E. Church, Sarah E. Warren, Yan Liang, Thomas H. Smith, Michael D. Bailey, James

Gowen-MacDonald: Employees, NanoString Technologies Inc., Seattle, WA, USA;

The other authors have no competing interests to disclose.

Patents

Bispecific CD123 $\times$ CD3 Diabodies for the Treatment of Hematologic Malignancies. Provisional application (Attorney Docket No. 1301.0161P3) filed 25 July 2019 and assigned Serial No. 62/878,368.

Data and materials availability

All data associated with this study are present in the paper or Supplementary Materials. Gene expression data have been deposited in NCBl's Gene Expression Omnibus (70) and are accessible through GEO Series accession number GSE134589/ 


\section{Figure Legends}

Fig. 1: Immune gene sets stratify bone marrow samples from patients with newly diagnosed AML. A) Unsupervised hierarchical clustering (Euclidean distance, complete linkage) of the correlation matrix of immune and biological activity signatures identifies coexpression patterns (grey boxes) of immune gene sets (correlation value color-coded per the legend, where blue denotes a Pearson correlation coefficient of -1.0 and red indicates a Pearson correlation coefficient of 1.0) in the bone marrow (BM) microenvironment of patients with AML (PMCC cohort; $n=290$ ), namely, IFN-dominant, adaptive and myeloid gene modules. Immune cell type (23) and signature scores (22) were calculated from mRNA expression as pre-defined linear combinations (weighted averages) of biologically relevant gene sets. Morpheus, an online tool developed at the Broad Institute (MA, USA) was used for data analysis and visualization. B) IFN-dominant, adaptive and myeloid scores in aggregate stratify patients with newly diagnosed AML into two distinct clusters, which are referred in this study as immune-infiltrated and immune-depleted (25). ClustVis, an online tool for clustering of multivariate data, was used for data analysis and visualization (71). C) Violin plots summarizing the expression of IFN-stimulated genes (ISGs), T-cell and cytotoxicity markers, negative immune checkpoints, genes implicated in antigen processing and presentation, and immunotherapy targets in AML cases with an immune-infiltrated and immune-depleted tumor microenvironment (TME). Data were compared using the Mann-Whitney $U$ test for unpaired data (two-sided). ${ }^{*} P<0.05 ;{ }^{* *} P<0.0001$. D) Spearman correlation coefficients between STAT1, ISGs [IRF1, MX1, IFIT1, TNFRSF14, PD-L1 (CD274)], surrogate markers for cytotoxic T cells (CD8A, GZMA) and negative immune checkpoints [LAG3, HAVCR2 (Tim-3)] under conditions of high and low immune infiltration.

Fig. 2: Multiplexed protein detection with GeoMx DSP identifies prognostic signatures in immune-infiltrated AML. A) CD3 hotspots (green fluorescence) in representative regions of interest (ROIs) from a bone marrow (BM) trephine biopsy obtained at time of AML diagnosis (SAL series). B) Association between PTEN and CD8/GZMB expression in geometric ROls 
$(n=240)$ from 10 BM FFPE sections (median split of PTEN barcode counts). Comparisons of CD8 and GZMB expression between PTEN ${ }^{\text {high }}$ and PTEN ${ }^{\text {low }}$ ROls were performed using the Mann-Whitney $U$ test for unpaired data (two-sided). C) Correlation matrix of protein expression in BM biopsies from 10 patients with newly diagnosed AML (SAL series). Protein expression data was subjected to unsupervised hierarchical clustering. Heatmaps were built using Morpheus with blue boxes denoting four distinct protein co-expression patterns (Pearson correlation coefficient $>0.45$ ) or signatures (SIG). D) Abnormalities in SIG3 genes (mRNA upregulation, gene amplification, deep deletion and mis-sense mutations, relative to the gene's expression distribution in all profiled AML samples) in TCGA cases. Data were retrieved, analyzed and visualized using cBioPortal. Abnormalities in only one gene utilized in the query (by default, non-synonymous mutations, fusions, amplifications and deep deletions) were sufficient to define that particular patient sample as "altered". E) GO enrichment analysis. For the gene list submitted to metascape.org, pathway and process enrichment analyses were carried out using all genes in the genome as the enrichment background. Terms with a $P$ value $<0.01$, a minimum count of 3 , and an enrichment factor $>1.5$ (defined as the ratio between the observed counts and the counts expected by chance) were collected and grouped into clusters based on their membership similarities. F) Heatmap of immune cell type-specific scores and biological activity scores in TCGA-AML cases with and without abnormalities (Alt.) of SIG3 genes. ClustVis was used for data analysis and visualization (71). G) Kaplan-Meier estimates of relapse-free survival (RFS) and overall survival (OS) in TCGA-AML patients with (red line) and without (blue line) abnormalities of SIG3 genes. HR = hazard ratio. Survival curves were compared using a log-rank (Mantel-Cox) test.

Fig. 3: Clinical correlates of immune profiles in patients with newly diagnosed AML. A) Stratification of patient survival (PMCC cohort; $n=290$ ) within each ELN cytogenetic risk category by immune subtype (immune-infiltrated and immune-depleted). Kaplan-Meier estimates of RFS and OS are shown. Survival curves were compared using a log-rank (Mantel-Cox) test. ${ }^{*} P<0.05 ;{ }^{* *} P<0.01$. HR=hazard ratio; $\mathrm{Cl}=$ confidence interval. B) 
Expression of PD1 and markers of $\mathrm{CD} 8^{+} \mathrm{T}$-cell exhaustion across cytogenetically defined patient categories. Data were compared using the Kruskal-Wallis test for unpaired

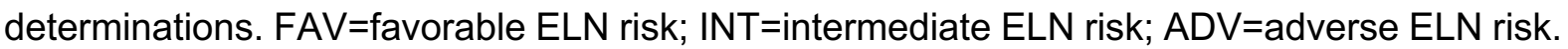
C) Cytogenetically defined categories stratify survival in patients with immune-infiltrated AML. Kaplan-Meier estimates of RFS and OS are shown. Survival curves were compared using a log-rank (Mantel-Cox) test. ${ }^{* *} P<0.001$. D) Molecularly defined categories stratify survival in ELN intermediate AML cases $(n=100)$ with immune-infiltrated and immune-depleted mRNA profiles. Patients were subclassified into molecular low risk (NPM1 mutations without FLT3ITD), molecular intermediate risk (NPM1 wild-type without FLT3-ITD or with low FLT3-ITD allelic ratio) and molecular high risk (NPM1 wild-type with FLT3-ITD), as previously reported (31). Kaplan-Meier estimates of OS are shown. Survival curves were compared using a logrank (Mantel-Cox) test. ${ }^{* * *} P<0.001$. E) AUROC curve measuring the predictive ability of molecular risk (blue curve) and immune subtype (red curve) for OS. SE=standard error; $\mathrm{Cl}=$ confidence interval. $\mathrm{AUROC}=1.0$ would denote perfect prediction and $\mathrm{AUROC}=0.5$ would denote no predictive ability.

Fig. 4: A 21-gene classifier stratifies survival in ELN-intermediate risk AML ( $n=100)$. A) Expression of the 21 differentially expressed (DE) genes across the PMCC discovery cohort (unsupervised hierarchical clustering; Euclidean distance; complete linkage). FDR=false discovery rate. Morpheus was used for data analysis and visualization. B) Expression of the 21 DE genes in patients with immune-infiltrated and immune-depleted AML. Data were compared using the Mann-Whitney $U$ test for unpaired determinations. Red bars indicate median values. C) DE genes between favorable and adverse-risk AML were mapped to gene ontology (GO) biological processes and pathways using Metascape.org. D) Kaplan-Meier estimate of RFS and OS in patients with ELN intermediate-risk AML stratified by the 21-gene classifier. Survival curves were compared using a log-rank (Mantel-Cox) test. HR=hazard ratio; $\mathrm{Cl}=$ confidence interval. ${ }^{* *} P<0.01 ;{ }^{* * *} P<0.001$. E) Kaplan-Meier estimate of OS in TCGA AML cases (first independent validation cohort) stratified by the 21-gene classifier 
(median split of gene expression values). Data were accessed, analyzed and visualized through the Gene Expression Profiling Interactive Analysis (GEPIA) portal (http://gepia2.cancer-pku.cn/\#survival) (72). Survival curves were compared using a log-rank (Mantel-Cox) test. F) Kaplan-Meier estimate of OS in HOVON AML cases (second independent validation cohort) stratified by the 21-gene classifier (median split of gene expression values). Survival curves were compared using a log-rank (Mantel-Cox) test. ${ }^{* * \star *} P$ $<0.0001$

Fig. 5: Differentially expressed immune genes across age groups and disease stages. A) Top 20 differentially expressed (DE) immune genes between childhood $(n=34)$ and adult AML cases $(n=46)$. ClustVis was used for data analysis and visualization. Violin plots summarize the expression of relevant chemokine genes. Data were compared using the Mann-Whitney $U$ test for paired determinations. Volcano plots of DE genes were generated using the nSolver software package. ${ }^{* * *} P<0.0001$. B) Top 20 DE immune genes between matched BM samples from 22 adult patients (SAL cohort) at diagnosis and achievement of complete remission (CR). Data were compared using the Mann-Whitney $U$ test for paired determinations. Volcano plots of DE genes were generated using the nSolver software package. ${ }^{* \star *} P<0.001$; ${ }^{* \star *} P<0.0001$. Data were compared using the Mann-Whitney $U$ test for paired determinations. C) Top 20 DE immune genes between matched BM samples from 22 adult patients (SAL cohort) at diagnosis and disease relapse. Data were compared using the Mann-Whitney $U$ test for paired determinations. Volcano plots of DE genes were generated using the nSolver software package. ${ }^{* *} P<0.01 ;{ }^{* *} P<0.001$. D) Venn diagram showing overlap in DE genes between children and adults with $\mathrm{AML}$, and patients at disease onset, achievement of CR and relapse.

Fig. 6: IFN-related mRNA profiles predict therapeutic resistance. A) Binary logistic regression predicting therapeutic response from IFN-related scores and conventional prognosticators, i.e., ELN cytogenetic risk category, WBC count at diagnosis, disease type 
(primary versus secondary AML), and patient age at diagnosis (PMCC discovery cohort). AUROC=area under receiver operating characteristic. The dotted line indicates currently accepted thresholds (>0.80) of AUROC with good predictive ability in AML (49). B) AUROC curves measuring the predictive ability of ELN cytogenetic risk and IFN-related scores for therapeutic response (PMCC discovery cohort; $n=290$ ). SE=standard error; $\mathrm{Cl}=$ confidence interval. $A U R O C=1.0$ would denote perfect prediction and $A U R O C=0.5$ would denote no predictive ability. C) AUROC curves measuring the predictive ability of ELN cytogenetic risk and IFN-related scores for therapeutic response in Beat AML trial specimens (validation cohort). SE=standard error; $\mathrm{Cl}=$ confidence interval. D) Unsupervised hierarchical clustering (Euclidean distance, complete linkage) of the correlation matrix of immune and biological activity signatures identifies co-expression patterns of immune gene sets (correlation value color-coded per the legend; Pearson correlation coefficient $>0.45$; blue boxes) in the bone marrow $(B M)$ microenvironment of AML patients in the HOVON series $(n=618$ cases with therapy response and ELN cytogenetic risk information). E) IFN-dominant, adaptive and myeloid scores in patients in the HOVON series. The Venn diagram shows the overlap between curated hallmark gene sets linked to IFN- $\gamma$ responses $(n=186)$ and inflammatory responses $(n=189)$. MSigDB=Molecular Signature Database. F) Gene set enrichment analysis (GSEA) plots representing the normalized enrichment score (NES) of hallmark IFN- $\gamma$ response genes, inflammatory response genes and a subset of overlapping genes $(n=36)$ between IFN- $\gamma$ and inflammatory gene sets in AML patients in the HOVON series who failed to respond to induction chemotherapy. Gene sets were downloaded from the MSigDB. Each run was performed with 1,000 permutations. FDR=false discovery rate.

Fig. 7: Immune subtypes associate with response to flotetuzumab immunotherapy. A) Unsupervised hierarchical clustering (Euclidean distance, complete linkage) of immune and biological activity signatures in the BM microenvironment of patients with relapsed/refractory AML $(n=30)$ receiving flotetuzumab immunotherapy in the CP-MGD006-01 clinical trial 
(NCT\#02152956). Anti-leukemic response was defined as detailed in Materials and Methods.

B) IFN-module and tumor inflammation signature (TIS) scores in baseline BM samples from patients with primary refractory and relapsed AML. Red dots denote patients with evidence of anti-leukemic activity of flotetuzumab. Horizontal lines indicate median values. Comparisons were performed using the Mann-Whitney $U$ test for unpaired data (two-sided). ${ }^{* *} P<0.01$. C) Area under receiver operating characteristic (AUROC) curves measuring the predictive ability of the IFN-module score and TIS scores for therapeutic response to flotetuzumab. $\mathrm{Cl}=$ confidence interval. D) Immune activation in the TME during flotetuzumab treatment (matched BM samples from 19 patients). Red dots denote patients with evidence of flotetuzumab anti-leukemic activity. Horizontal lines indicate median values. Comparisons were performed with the Mann-Whitney $U$ test for paired data (two-sided). Pre=baseline. C1=cycle 1. ${ }^{* *} P<0.01 .{ }^{* *} P<0.001$. E) Principal component analysis (PCA) of GeoMx DSP housekeeping-normalized barcode counts for 52 proteins from 11 pre-treatment and 8 matched post-cycle 1 (C1) BM samples with 5-35 regions of interest (ROls) profiling the entire FFPE biopsy (Fig. S12). Points are colored by no response (NR; pink) or complete response (CR; green). F) Identification of CD3 hotspots in ROls from a BM biopsy of a representative patient who achieved CR after flotetuzumab immunotherapy. $\mathrm{CD}^{+} \mathrm{T}$ cells are shown in yellow. G) Differential expression of immuno-oncology (IO)-related proteins between baseline and post-C1 FFPE BM biopsies from two patients achieving CR after flotetuzumab immunotherapy. Analysis was performed using a linear mixed effect model. Vertical dotted lines represent $\pm 0.5 \log 2$ fold change (FC) and the horizontal dotted line indicates a $P$ value of 0.05. NS=not significant. H) Differential expression of IO-related proteins between ROls with or without CD3 hotspots in two patient samples after 1 cycle of flotetuzumab who achieved CR. Analysis was performed using a linear mixed effect model. Vertical dotted lines represent $\pm 0.5 \log 2$ fold-change and the horizontal dotted line indicates a $P$ value of 0.05 . 
Table 1. AML cohorts selected for targeted immune gene expression profiling

\begin{tabular}{|c|c|c|c|}
\hline Patient series & PMCC & CHOP & SAL \\
\hline \# of patients & 290 & 34 & 46 \\
\hline Age (0-39), $n$ & 76 & 34 & 13 \\
\hline Age (40-59), $n$ & 126 & 0 & 19 \\
\hline $\operatorname{Age}(\geq 60), n$ & 88 & 0 & 14 \\
\hline Median age (years, range) & $52(18-81)$ & $10(0.1-20)$ & $53.5(23-75)$ \\
\hline WBC count at presentation & $\begin{array}{l}19.15 \times 10^{3} / \mu \mathrm{L} \\
(0.7-399)\end{array}$ & N.A. & $\begin{array}{c}56.45 \times 10^{3} / \mu \mathrm{L} \\
(0.84-320.2)\end{array}$ \\
\hline $\begin{array}{r}\text { Cytogenetic risk group, } n \\
\text { ELN favorable } \\
\text { ELN intermediate } \\
\text { ELN adverse } \\
\text { N.A. }\end{array}$ & $\begin{array}{c}35 \\
155 \\
59 \\
41\end{array}$ & $\begin{array}{c}8 \\
20 \\
6 \\
-\end{array}$ & $\begin{array}{c}10 \\
27 \\
4 \\
5\end{array}$ \\
\hline $\begin{array}{l}\text { Disease status at time of BM } \\
\text { sampling, } n \\
\text { Complete remission } \\
\text { Relapse }\end{array}$ & $\begin{array}{c}290 \\
0 \\
0\end{array}$ & $\begin{array}{l}34 \\
16 \\
11\end{array}$ & $\begin{array}{l}46 \\
21 \\
24\end{array}$ \\
\hline \# of BM samples analyzed & 290 & 61 & 91 \\
\hline $\begin{array}{l}\text { \# of BM samples analyzed using } \\
\text { the GeoMx DSP platform }\end{array}$ & 0 & 0 & 10 \\
\hline $\begin{array}{l}\text { De novo/secondary/therapy- } \\
\text { related }\end{array}$ & $244 / 46 / 0$ & $36 / 4 / 0$ & $39 / 5 / 2$ \\
\hline $\begin{array}{l}\text { Response to induction } \\
\text { chemotherapy } \\
\text { Yes } \\
\text { No } \\
\text { Primary induction failure } \\
\text { N.A. }\end{array}$ & $\begin{array}{c}210 \\
80 \\
39 \\
-\end{array}$ & $\begin{array}{c}26\left(\mathrm{M} 1^{*}\right) \\
3\left(\mathrm{M} 2^{*}\right) \\
2 \\
5\end{array}$ & $\begin{array}{c}33 \\
4 \\
2 \\
3\end{array}$ \\
\hline $\begin{array}{l}\text { Relapse } \\
\qquad \begin{array}{r}\text { Yes } \\
\text { No } \\
\text { N.A. }\end{array}\end{array}$ & $\begin{array}{c}132 \\
118 \\
1\end{array}$ & $\begin{array}{c}18 \\
15 \\
1\end{array}$ & $\begin{array}{c}31 \\
15 \\
0\end{array}$ \\
\hline Median follow-up time (months) & 101.23 & 70.2 & 55.54 \\
\hline Median RFS (months) & 19.1 & 25.6 & 13.64 \\
\hline Median OS (months) & 21.37 & 66.8 & 50.58 \\
\hline
\end{tabular}

Legend: N.A. $=$ Not available; $\mathrm{PMCC}=$ Princess Margaret Cancer Centre $; \mathrm{CHOP}=$ Children's Hospital of Philadelphia; SAL = Studienallianz Leukämie; ELN = European Leukemia-Net; BM = bone marrow; $\mathrm{DSP}=$ digital spatial profiling; N.A. = not available. Median follow-up time was calculated using the reverse Kaplan-Meier method, with the event indicator reversed so that the outcome of interest becomes censored. * $\mathrm{M} 1, \mathrm{M} 2$ and $\mathrm{M} 3 \mathrm{BM}$ remission status was defined as $<5 \%, 5 \%$ to $24 \%$ and $>25 \%$ AML blasts after induction chemotherapy, respectively (73). RFS $=$ relapse-free survival; OS = overall survival. 


\section{Supplementary Figures}
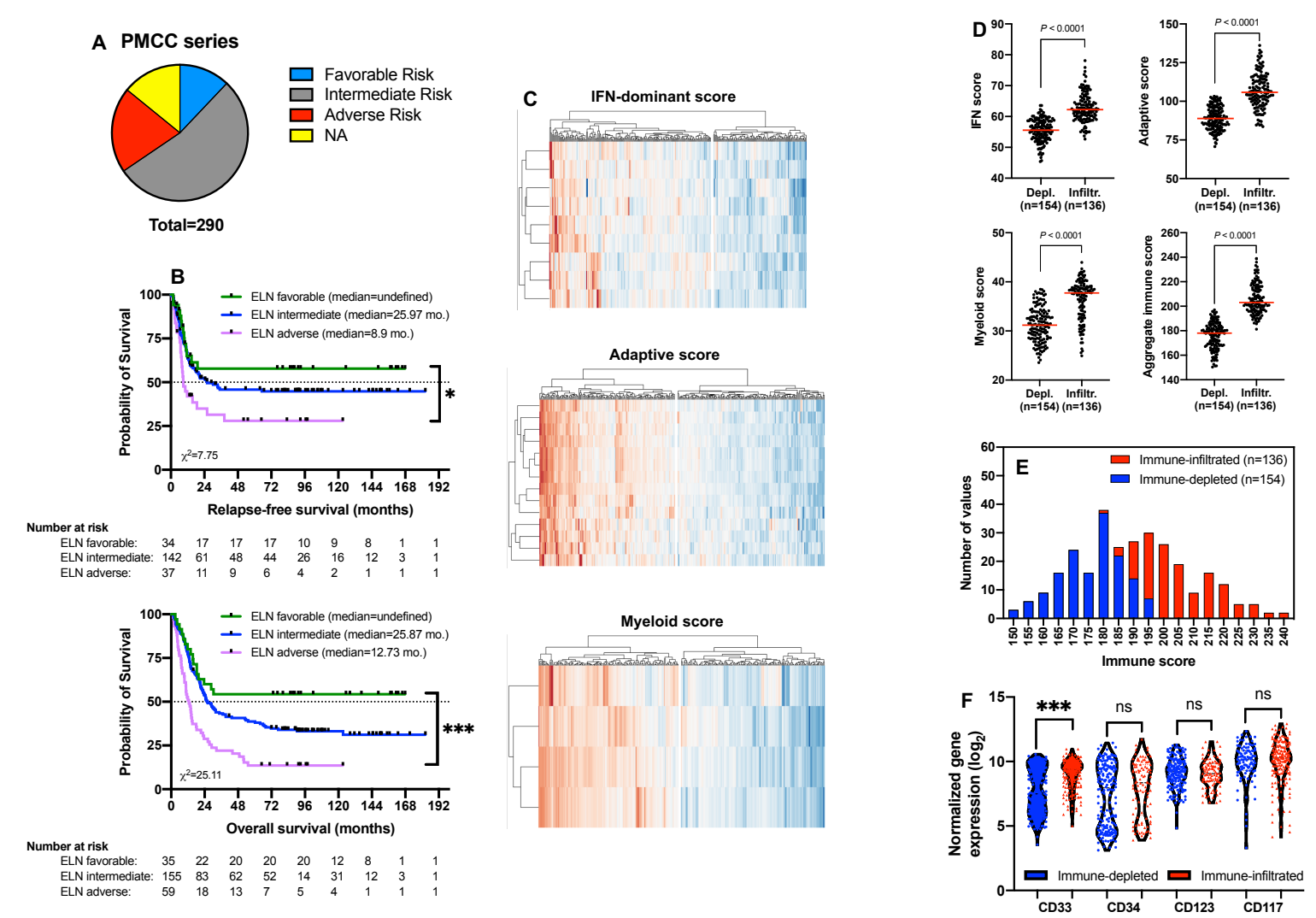

Fig. S1: Immune gene signatures and survival in the AML discovery series. A) Pie chart showing patient distribution by European Leukemia Net (ELN) cytogenetic risk category in the PMCC cohort ( $n=290$ cases). NA = not available. B) Cohort-wide relapse-free survival (RFS) and overall survival (OS); disease and patient characteristics are detailed in Table 1. The Kaplan-Meier method was used to generate survival curves, which were compared using a log-rank test $\left({ }^{*} p<0.05 ;{ }^{* * *} p<0.001\right)$. Tick marks indicate censored observations. C) Interferon (IFN), adaptive, and myeloid signature scores individually separate AML cases according to high and low expression values. ClustVis, an online tool for clustering of multivariate data, was used for data analysis and visualization. D) Immune scores in the immune-depleted and immune-infiltrated subgroups. Data were compared using the Mann-Whitney $U$ test for unpaired determinations. Red bars indicate median values. E) Distribution of the aggregate immune score in patients with immune-infiltrated and immune-depleted AML. F) Violin plots summarizing the expression of leukemia-associated markers (CD33, CD34, CD123, CD117) in immune-infiltrated and immune-depleted AML cases from the PMCC cohort. Data were compared using the Mann-Whitney $U$ test for unpaired determinations $\left({ }^{*} p<0.05 ;{ }^{* * *} p<0.001\right.$; ns=not significant). E) Kaplan-Meier estimate of RFS in patients with AML separated by immune gene score quartiles (highest $[n=78]$ and lowest $[n=79]$ ). Survival curves were compared using the log-rank test $\left({ }^{*} p<0.05 ;{ }^{* *} p<0.01\right)$. 


\section{SAL series}

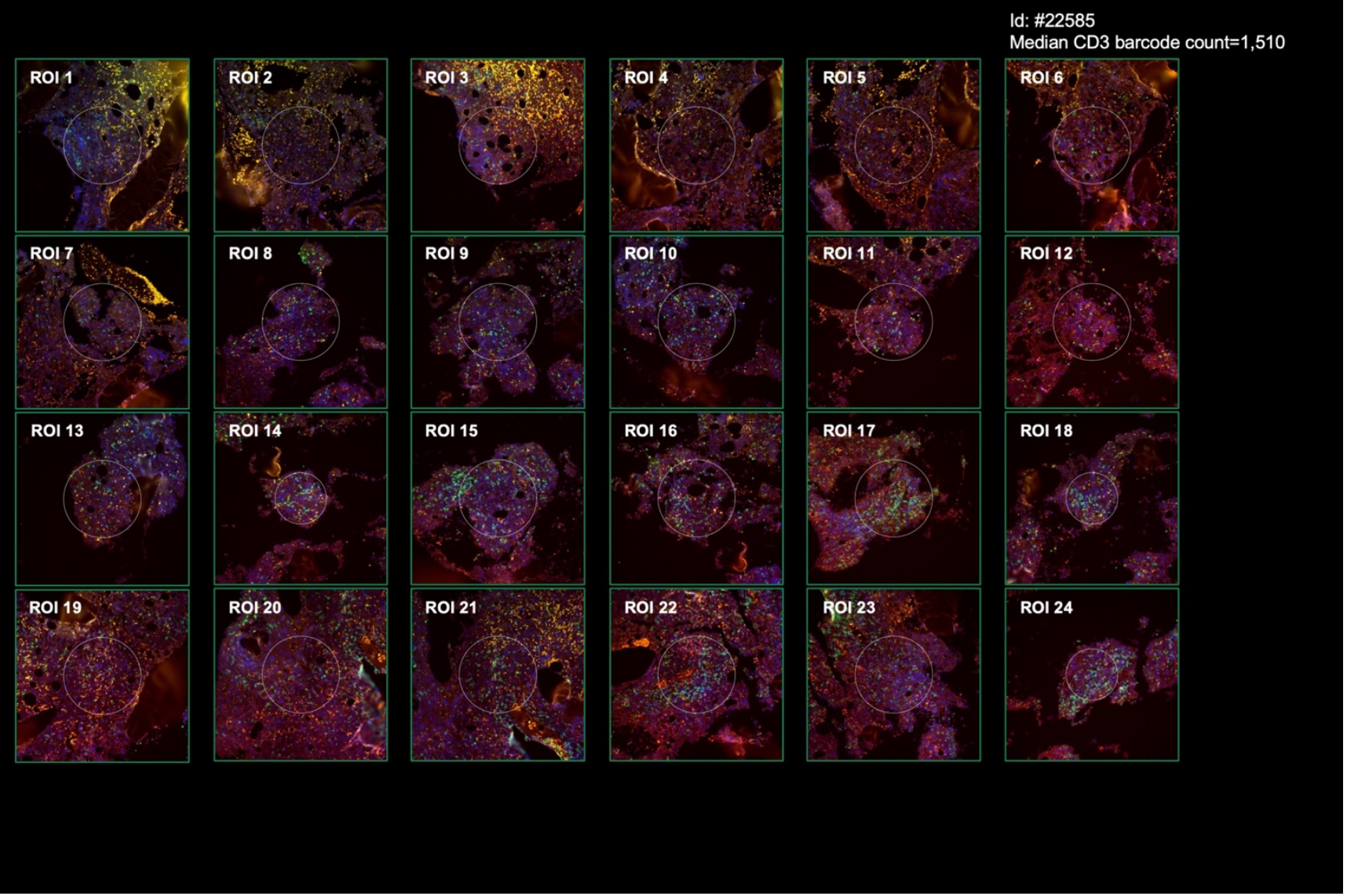

Fig. S2: GeoMx digital spatial profiling (DSP) and region of interest (ROI) selection in a representative pre-treatment BM trephine biopsy (SAL series) with high T-cell infiltration. CD3 staining is shown in green. The median CD3 barcode count (24 ROls) is indicated. 


\section{SAL series}

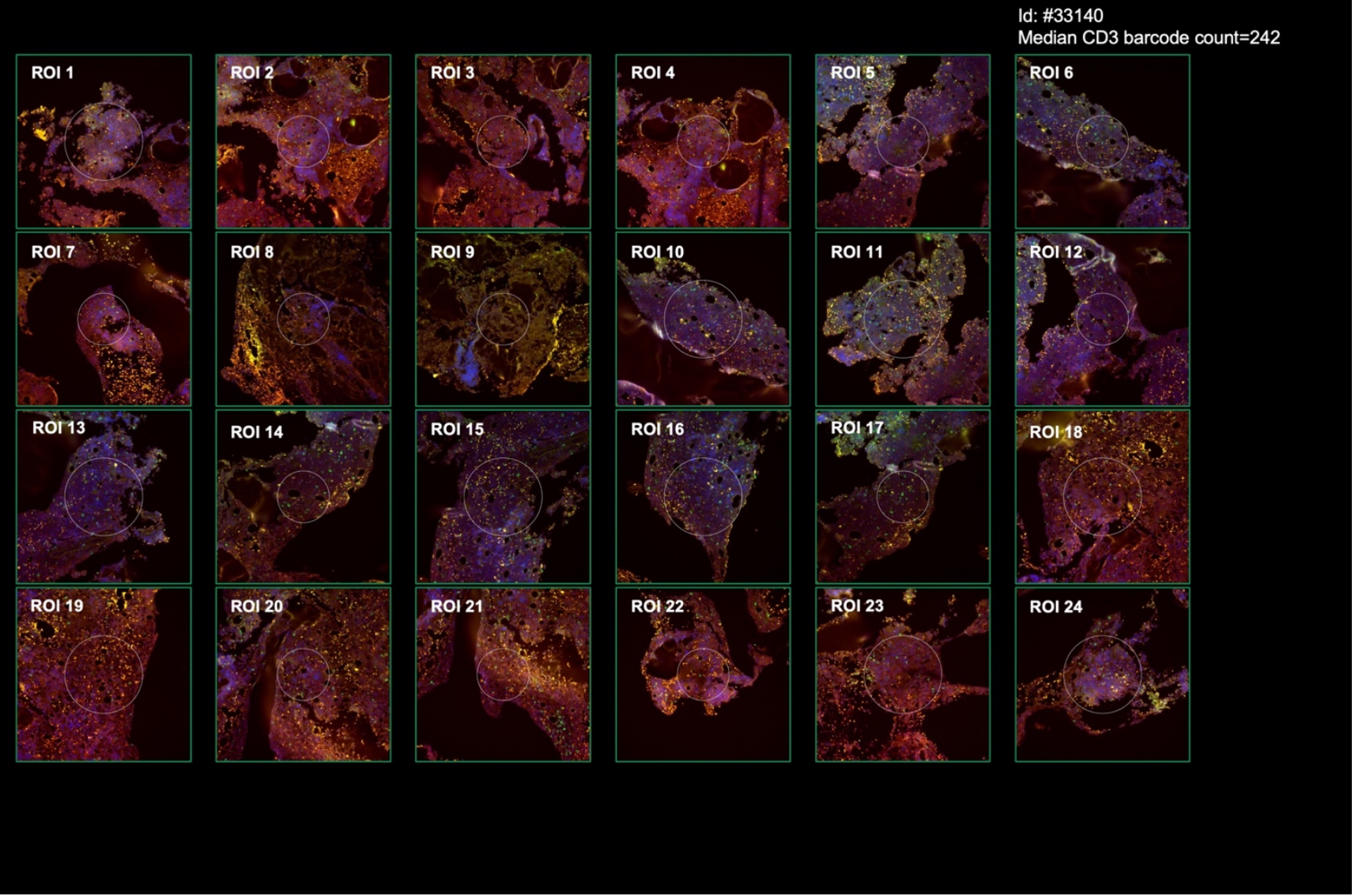

Fig. S3: GeoMx digital spatial profiling (DSP) and region of interest (ROI) selection in a representative pre-treatment BM trephine biopsy (SAL series) with low T-cell infiltration. CD3 staining is shown in green. The median CD3 barcode count (24 ROls) is indicated. 


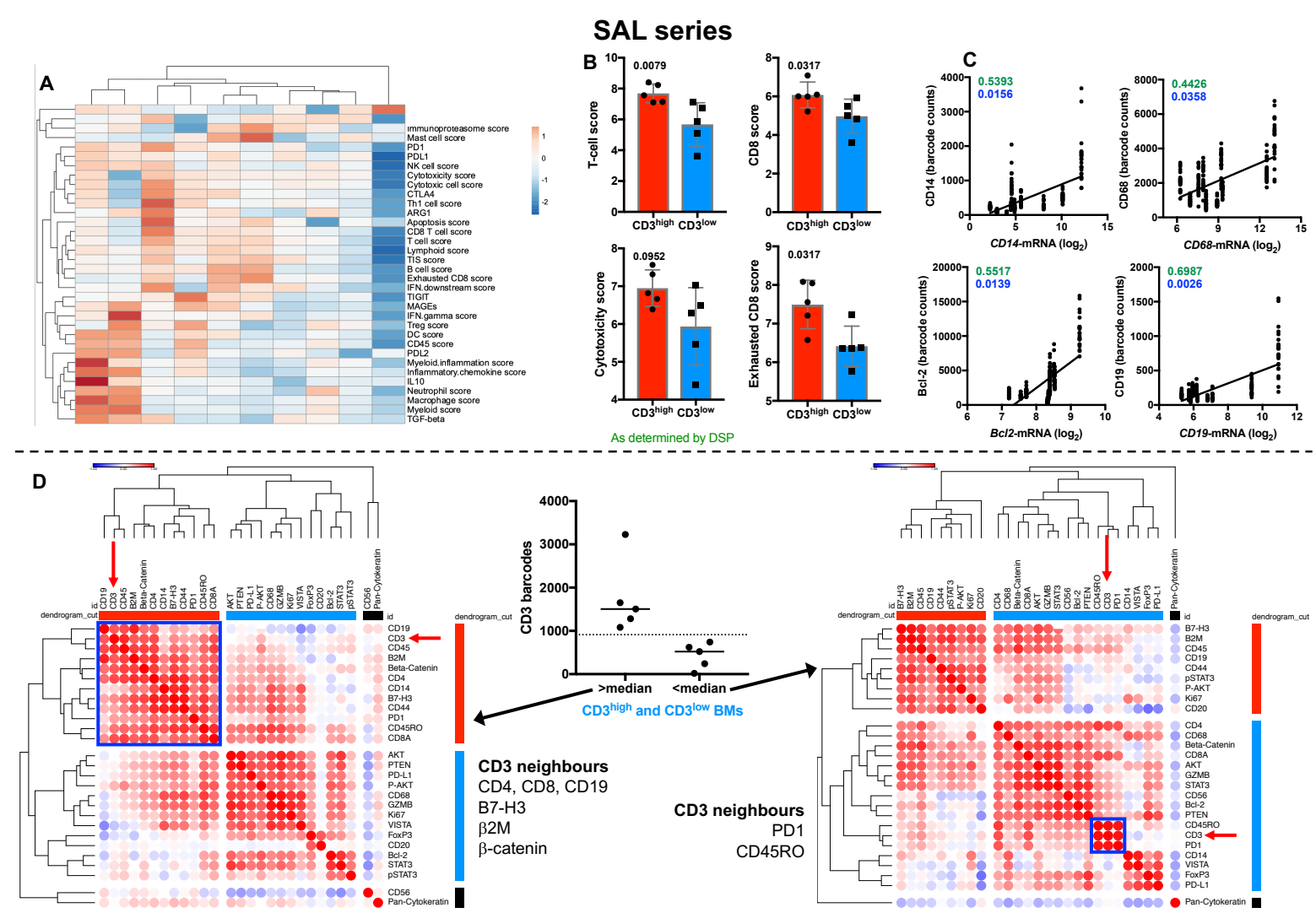

Fig. S4: Highly multiplexed protein profiling in the SAL patient cohort. A) Unsupervised hierarchical clustering (Euclidean distance, complete linkage) of immune cell type-specific and biological activity scores (mRNA measurements using the PanCancer Immune Profiling panel) in 10 pre-treatment BM trephine biopsies used for GeoMx DSP. ClustVis, an online tool for clustering of multivariate data, was used for data analysis and visualization. B) Association between CD3 protein expression, as determined by GeoMx DSP, and mRNA immune gene signatures (T-cell score, CD8 score, exhausted CD8 score, NK score, cytotoxicity score and macrophage score) as measured with the $\mathrm{nCounter} \mathrm{platform.} \mathrm{Data} \mathrm{were} \mathrm{compared} \mathrm{using} \mathrm{the}$ Mann-Whitney $U$ test for unpaired determinations. C) Correlation between mRNA and protein expression, as determined by GeoMx DSP. Spearman rank correlation coefficients are shown in green; $p$ values are shown in blue. D) Unsupervised hierarchical clustering (Euclidean distance, complete linkage) of the correlation matrix of immuno-oncology proteins detected with GeoMx Digital Spatial Profiling (DSP) allows the identification of CD3 neighbors in 10 pretreatment BM trephine biopsies from adult patients with high and low T-cell infiltration (median split). $\beta 2 \mathrm{M}=\beta 2$-microglobulin. Morpheus, an online tool developed at the Broad Institute (MA, USA) was used for data analysis and visualization. 


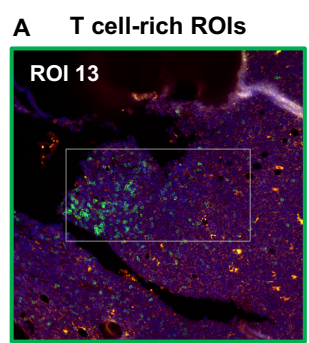

T cell-poor ROls
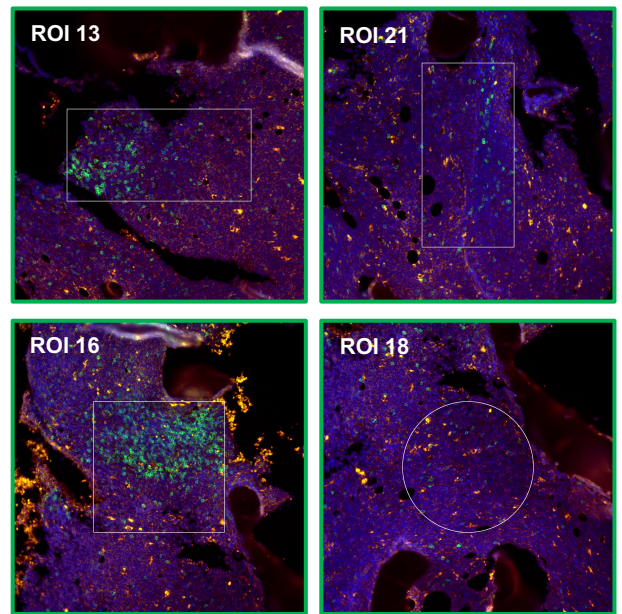

ROI 18

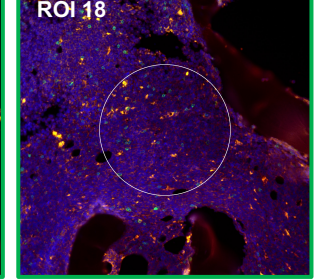

$\mathrm{CD}^{++}=$Top $25 \%$ quartile (cohort-wide) $\mathrm{CD}^{-/+}=$Bottom $25 \%$ quartile (cohort-wide)

C
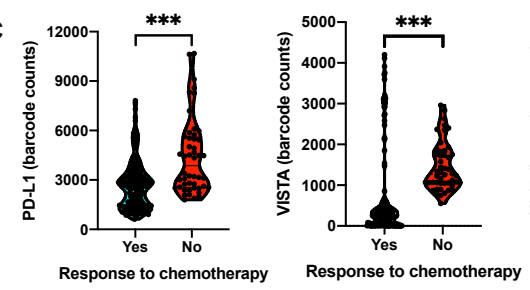
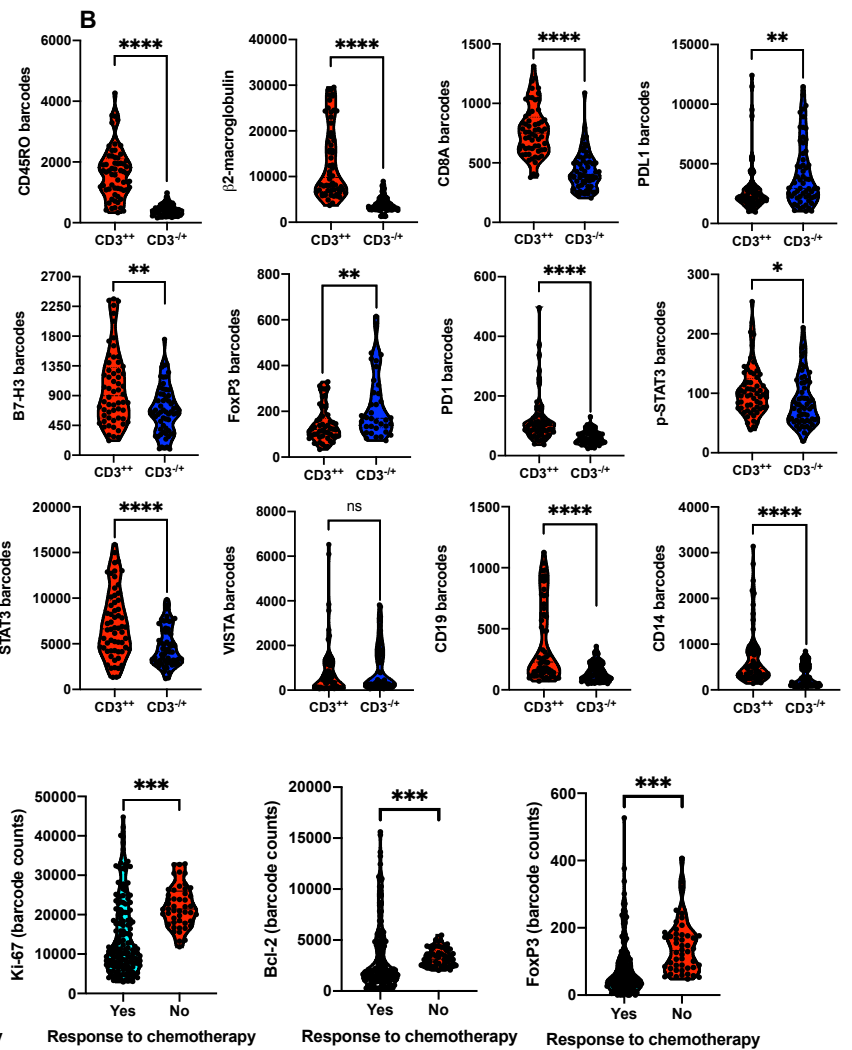

Fig. S5: Correlation between CD3 infiltration and expression of immuno-oncology (IO)related proteins as revealed by GeoMx digital spatial profiling (DSP) of BM trephine biopsies (SAL series). A) CD3 staining is shown in green. ROls $(n=24)$ were assigned to either the T-cell-rich (CD3 "hotspot"; top 25\% quartile of CD3 barcode counts) or the T-cellpoor category (bottom 25\% quartile of CD3 barcode counts). B) Violin plots showing expression of CD8, 32 -microglobulin, activation marker CD45RO, B7-H3, PD1, total and phosphorylated STAT3, CD14 and CD19 in relation to CD3 infiltration. Data were compared using the Mann-Whitney $U$ test for unpaired determinations $\left({ }^{*} P<0.05\right.$; ${ }^{* *} P<0.01$; ${ }^{* * * *} P<0.0001$; ns=not significant). C) Violin plots showing the association between protein expression and response to induction chemotherapy, which was defined as either morphologic complete remission (CR) or early blast clearance ( $<10 \%$ blasts on day 16 , i.e., one week after the end of induction chemotherapy), as previously published $(45,74)$. Data were compared using the Mann-Whitney $U$ test for unpaired determinations $\left({ }^{* * *} P<0.001\right)$. 

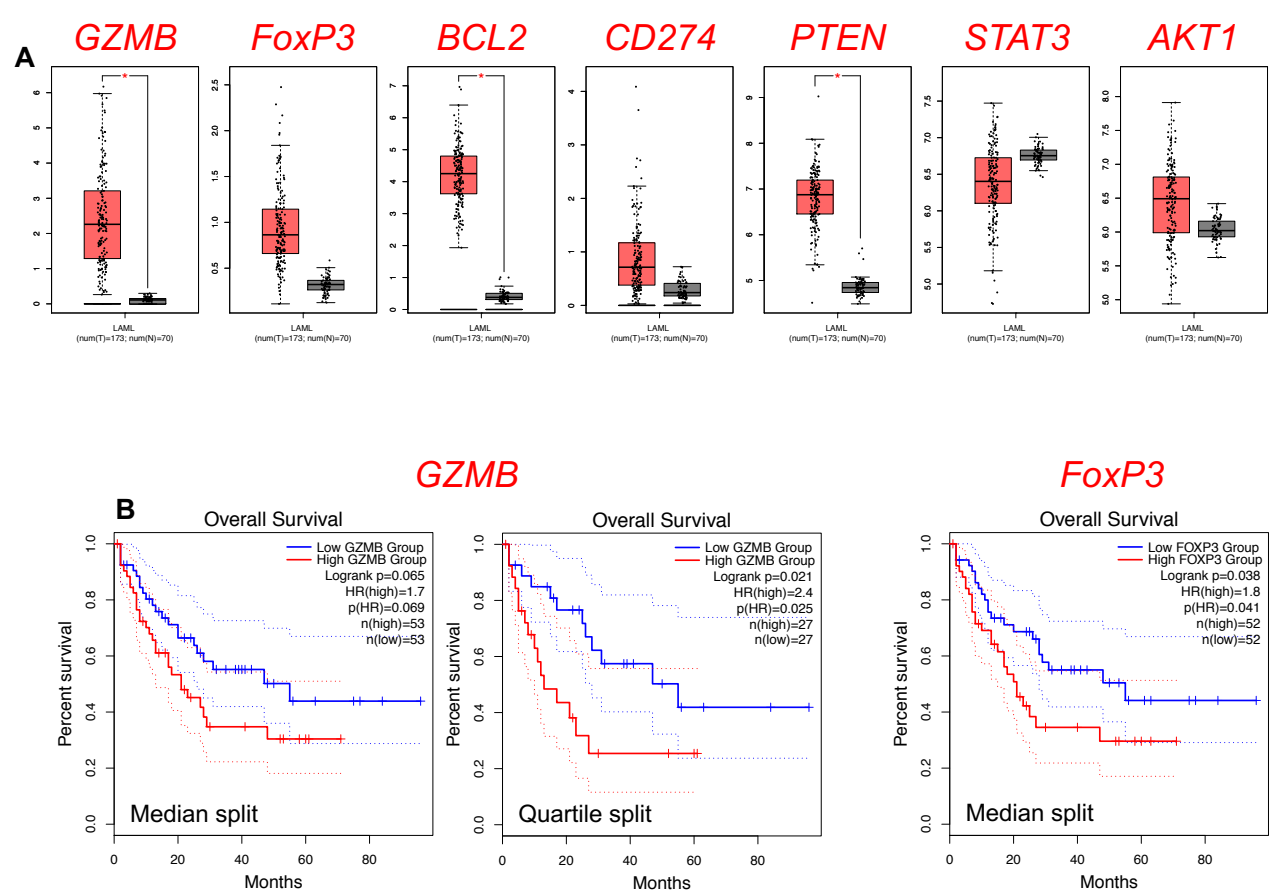

GZMB
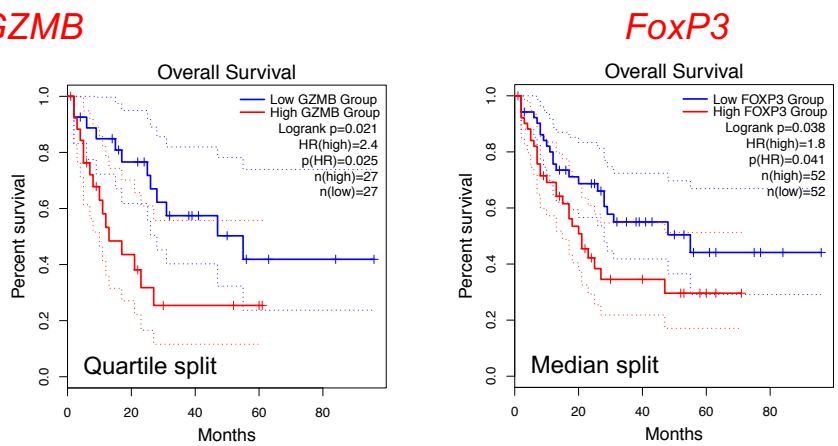

Fig. S6: Expression of SIG3 genes in TCGA-AML cases and in healthy tissues. A) Comparison of SIG3 gene expression in TCGA-AML cases $(n=173)$ and in normal blood ( $n=70$; Genotype Tissue Expression [GTEx] samples). Data were accessed and analyzed using GEPIA2 (http://gepia2.cancer-pku.cn/\#index). B) GZMB and FoxP3 survival predictions. A median split and/or quartile split of gene expression scores was used to compute KaplanMeier curves. 

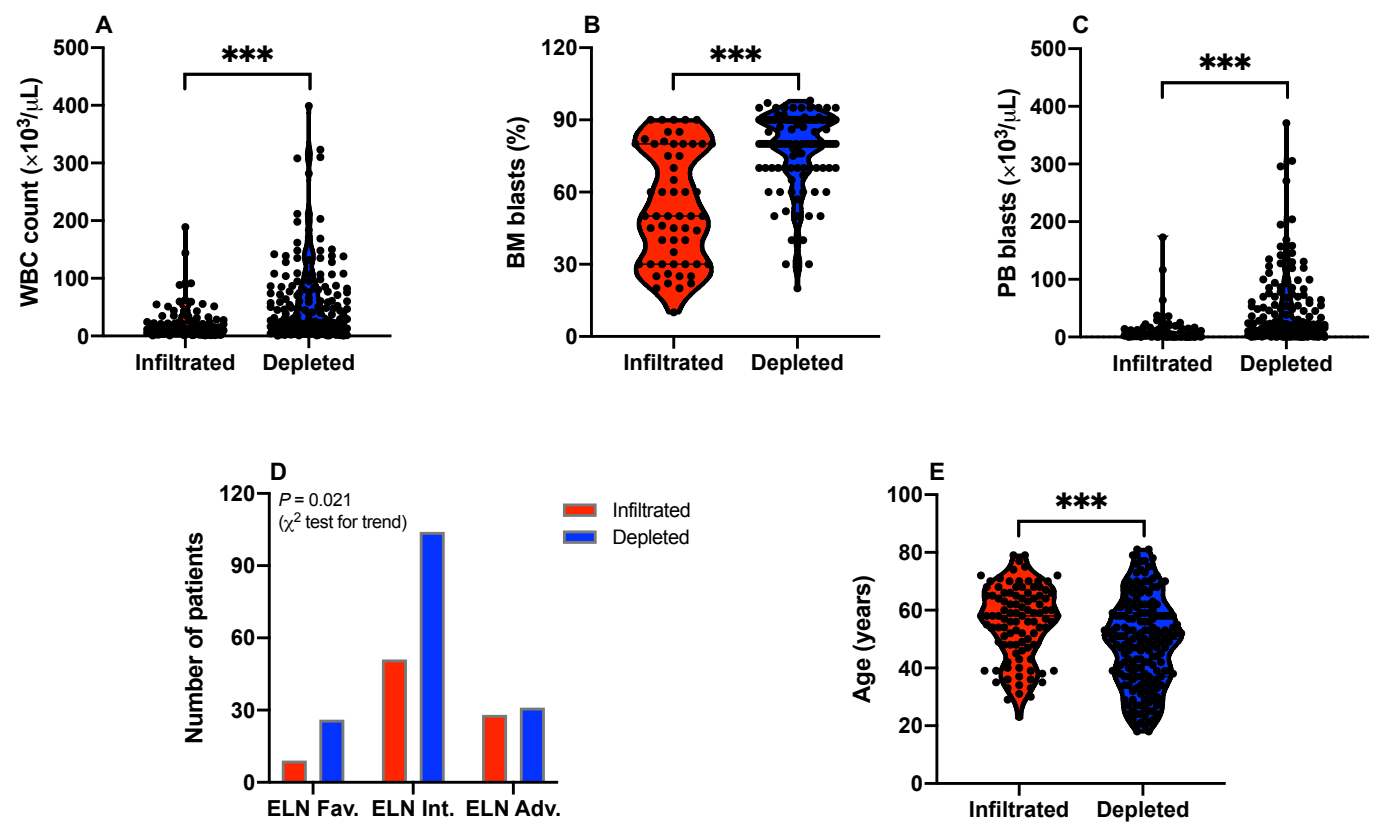

Fig. S7: Association between immune gene signatures and patients' characteristics in the AML discovery series. Violin plots summarizing white blood cell (WBC) count $(A)$, percentage of bone marrow (BM) blasts (B) and peripheral blood (PB) blasts (C), ELN cytogenetic risk category (D) and age at diagnosis (E) in immune-infiltrated and immunedepleted patients (PMCC cohort; $n=290$ cases). Immune gene signatures were derived as detailed in Fig. 1. Comparisons were performed using the Mann-Whitney $U$ test for unpaired determinations. ${ }^{*} P<0.05 ;{ }^{* * *} P<0.001$. 


\section{PMCC series}

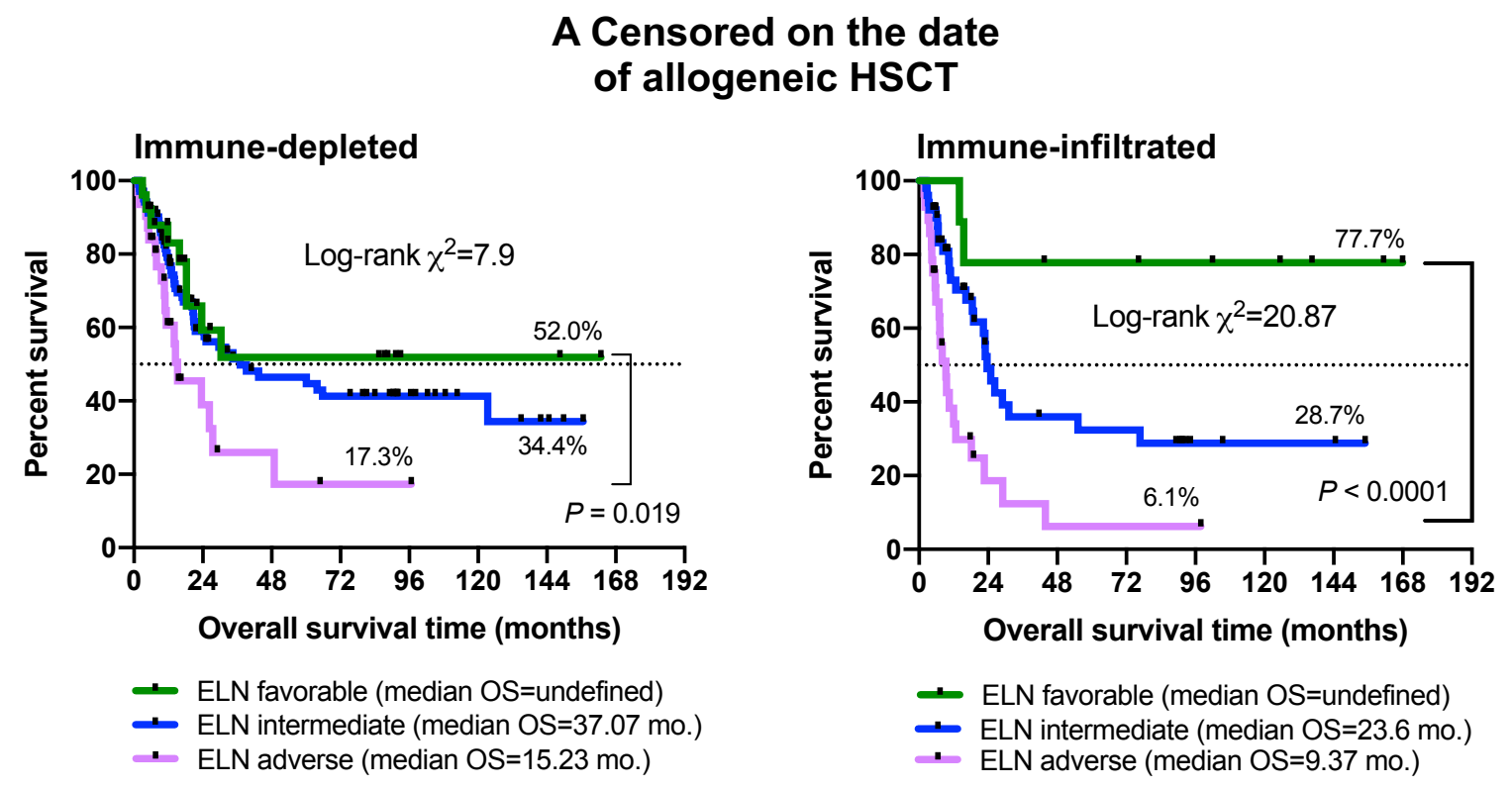

B Immune-infiltrated AML

\section{Chemotherapy}

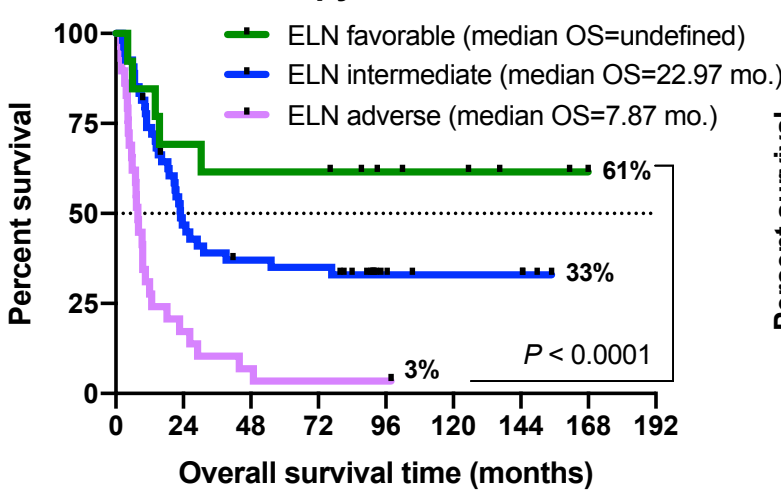

Allogeneic HSCT

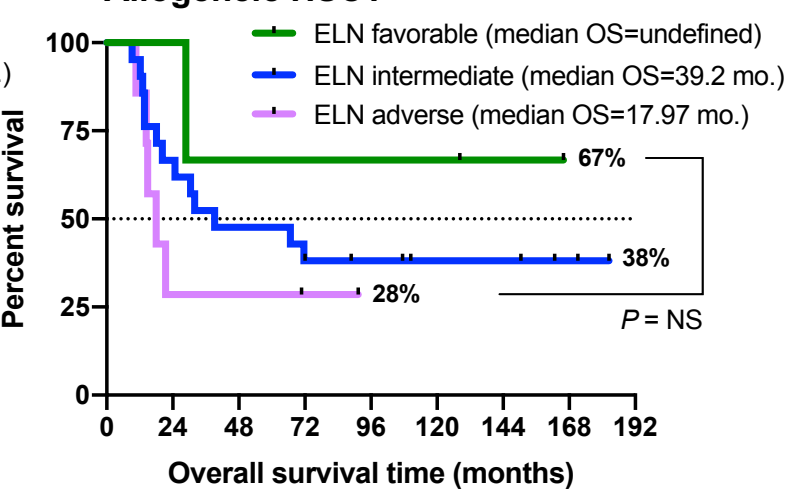

\section{Immune-depleted AML}

\section{Chemotherapy}

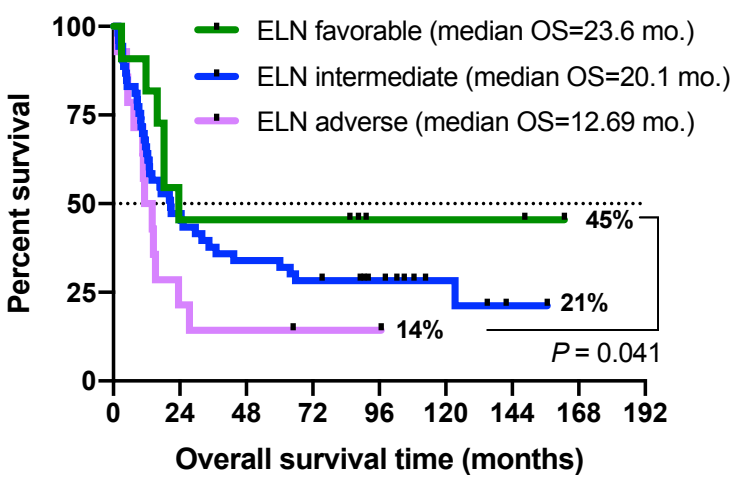

Allogeneic HSCT

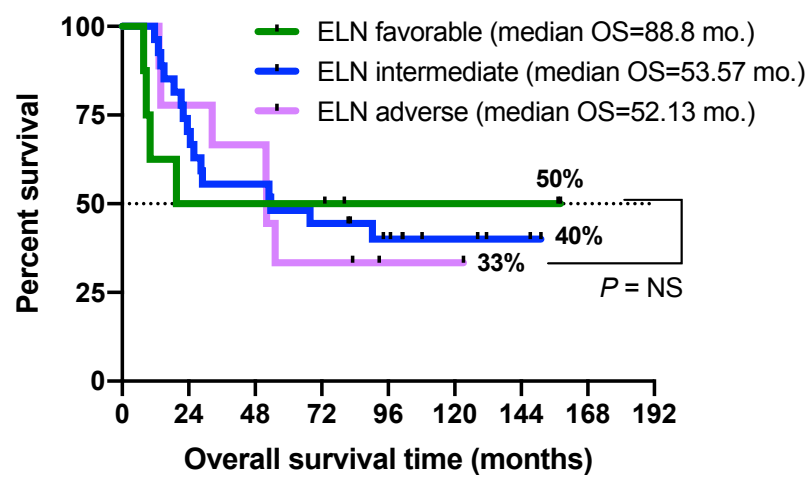

Fig. S8: Clinical outcomes of patients with immune-infiltrated and immune-depleted AML treated with allogeneic HSCT (PMCC cohort). A) Kaplan-Meier estimate of overall 
survival (OS) in patients with immune-infiltrated and immune-depleted AML censored on the date of allogeneic HSCT. Survival curves were compared using a log-rank (Mantel-Cox) test. B) Kaplan-Meier estimate of overall survival (OS) in patients with immune-infiltrated AML treated with chemotherapy either alone or in combination with allogeneic HSCT. Patients were stratified based on ELN risk. Survival curves were compared using a log-rank (Mantel-Cox) test. C) Kaplan-Meier estimate of overall survival in patients with immune-depleted AML treated with chemotherapy either alone or in combination with allogeneic HSCT. Patients were stratified based on ELN risk. Survival curves were compared using a log-rank (Mantel-Cox) test. 


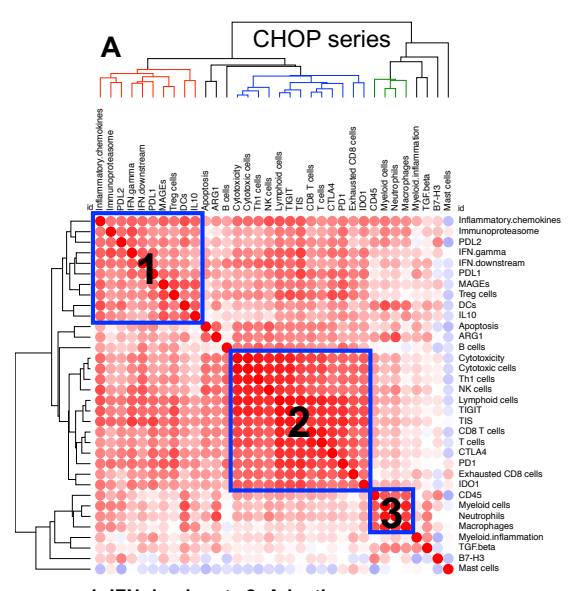

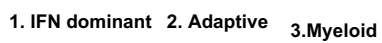

B

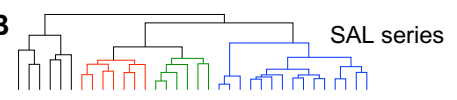

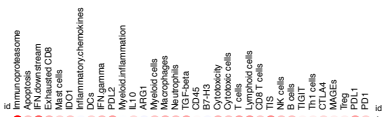

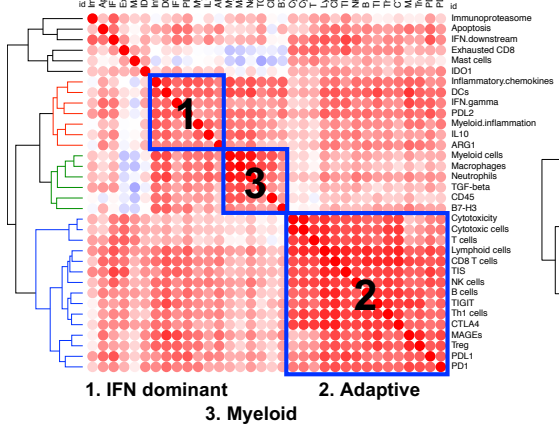

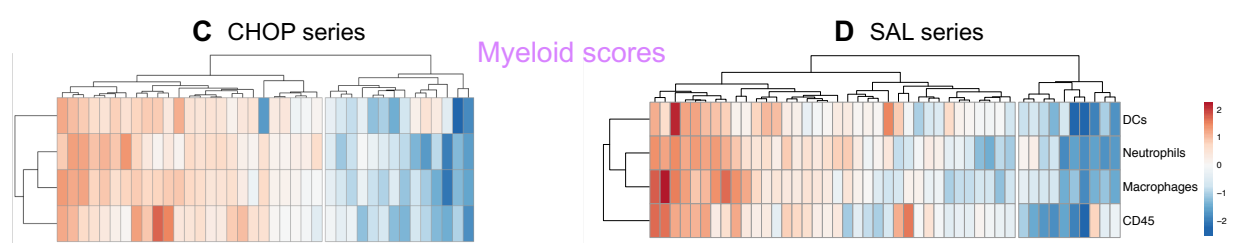

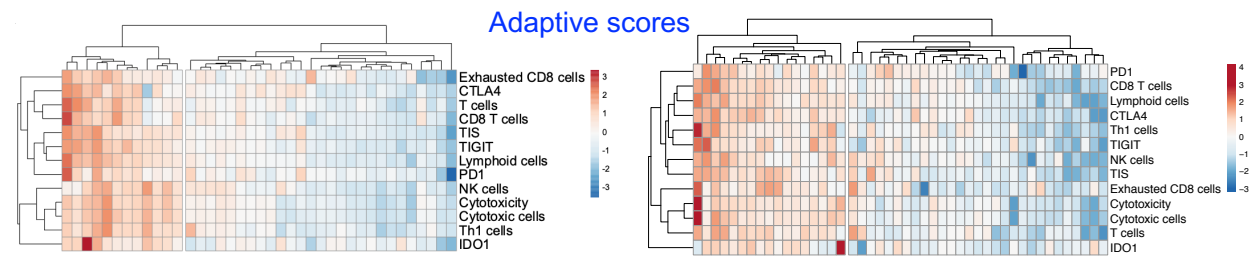

IFN scores
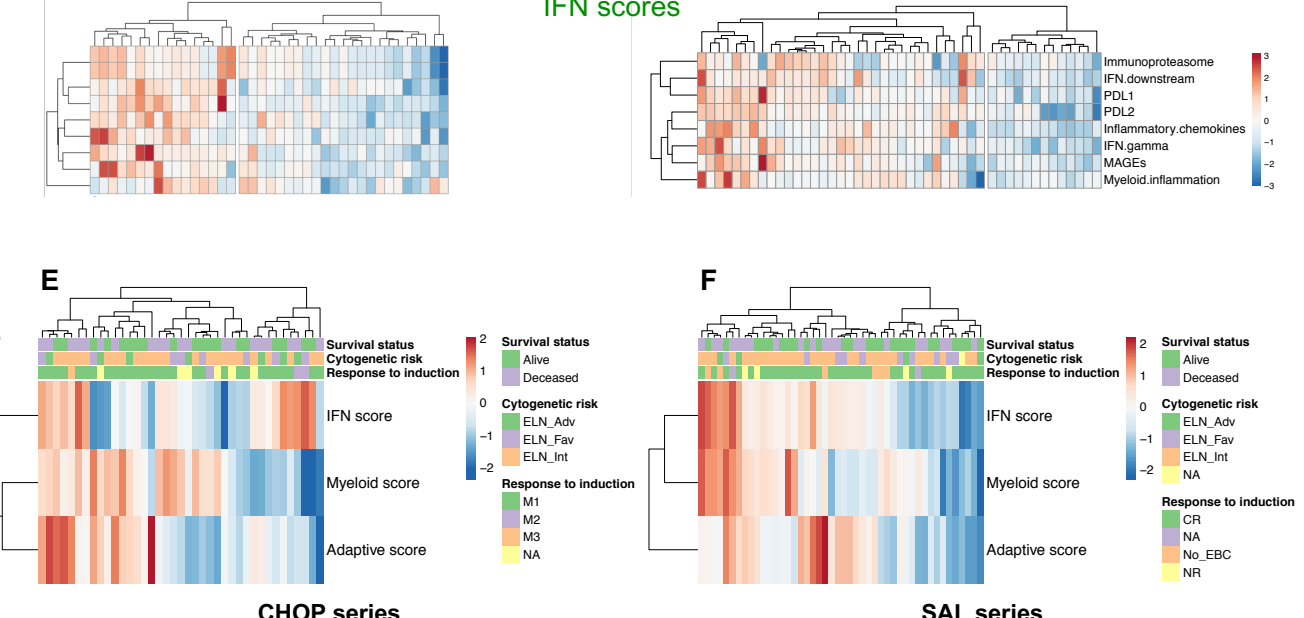

SAL series

Fig. S9: Immune gene signatures in the CHOP and SAL cohorts. A-B) Unsupervised hierarchical clustering (Euclidean distance, complete linkage) of the correlation matrix of immune and biological activity signatures highlights co-expression patterns of immune gene sets (Pearson correlation coefficient $>0.45$; blue boxes) in diagnostic bone marrow (BM) samples from children (CHOP cohort; $n=34$ ) and adults with AML (SAL cohort; $n=46$ ), namely, IFN-dominant signatures, adaptive immunity signatures and myeloid signatures. Morpheus was used for data analysis and visualization. C-D) Gene signatures in the IFN, adaptive, and myeloid scores stratify children and adults with AML (CHOP and SAL series) into subgroups with high and low expression. ClustVis was used for data analysis and visualization. E-F) IFN, adaptive, and myeloid scores in aggregate stratify children and adults with AML (CHOP and SAL series) into immune-infiltrated and immune-depleted subgroups. ClustVis was used for data analysis and visualization. ELN = 2017 European Leukemia-Net. Criteria for response to induction chemotherapy (M1 through $\mathrm{M} 3$ in the top panel and early blast clearance $[E B C] /$ complete remission $[C R]$ in the bottom panel) are detailed in Table 1. ADV=adverse; INT=intermediate; FAV=favorable; $N A=$ not available; $N R=$ no response. 

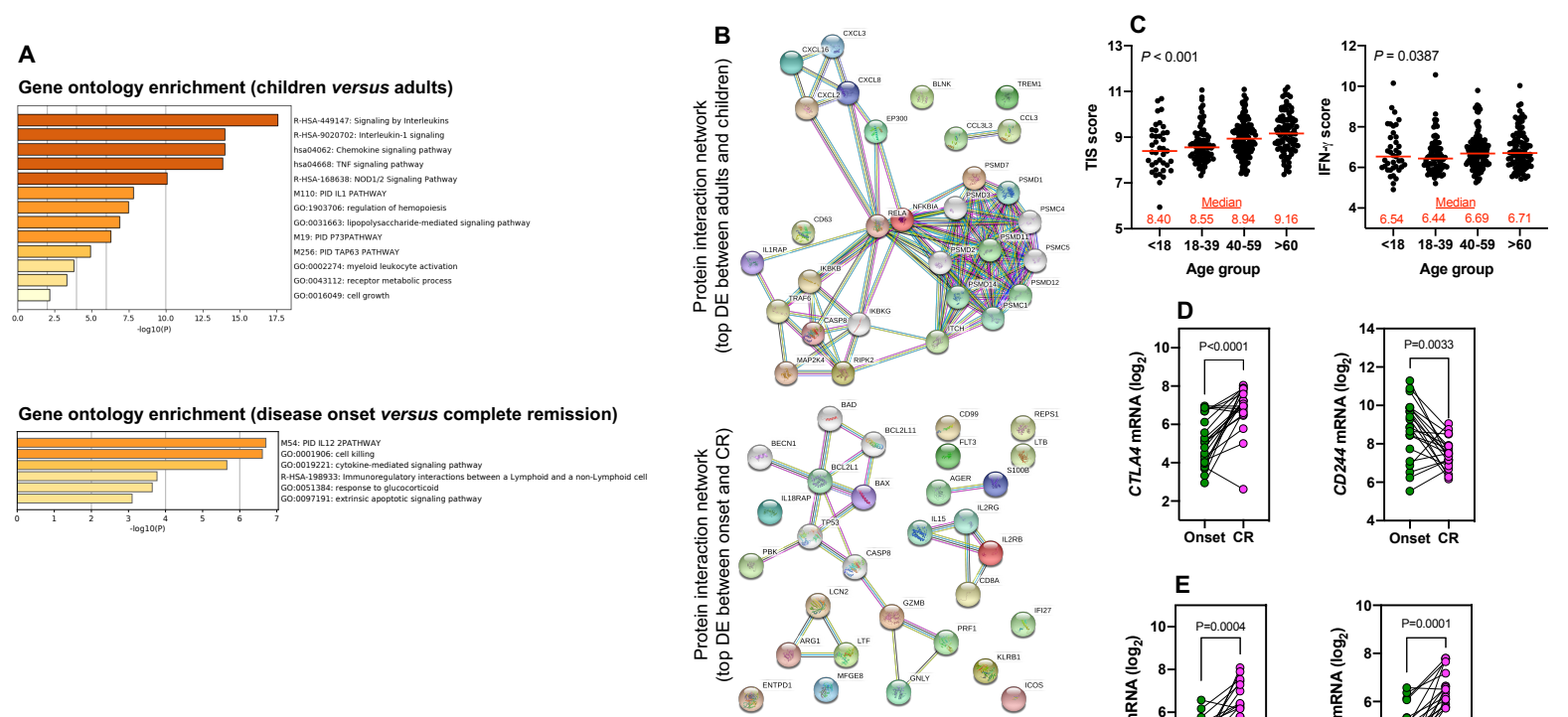

Gene ontology enrichment (disease onset versus relapse)
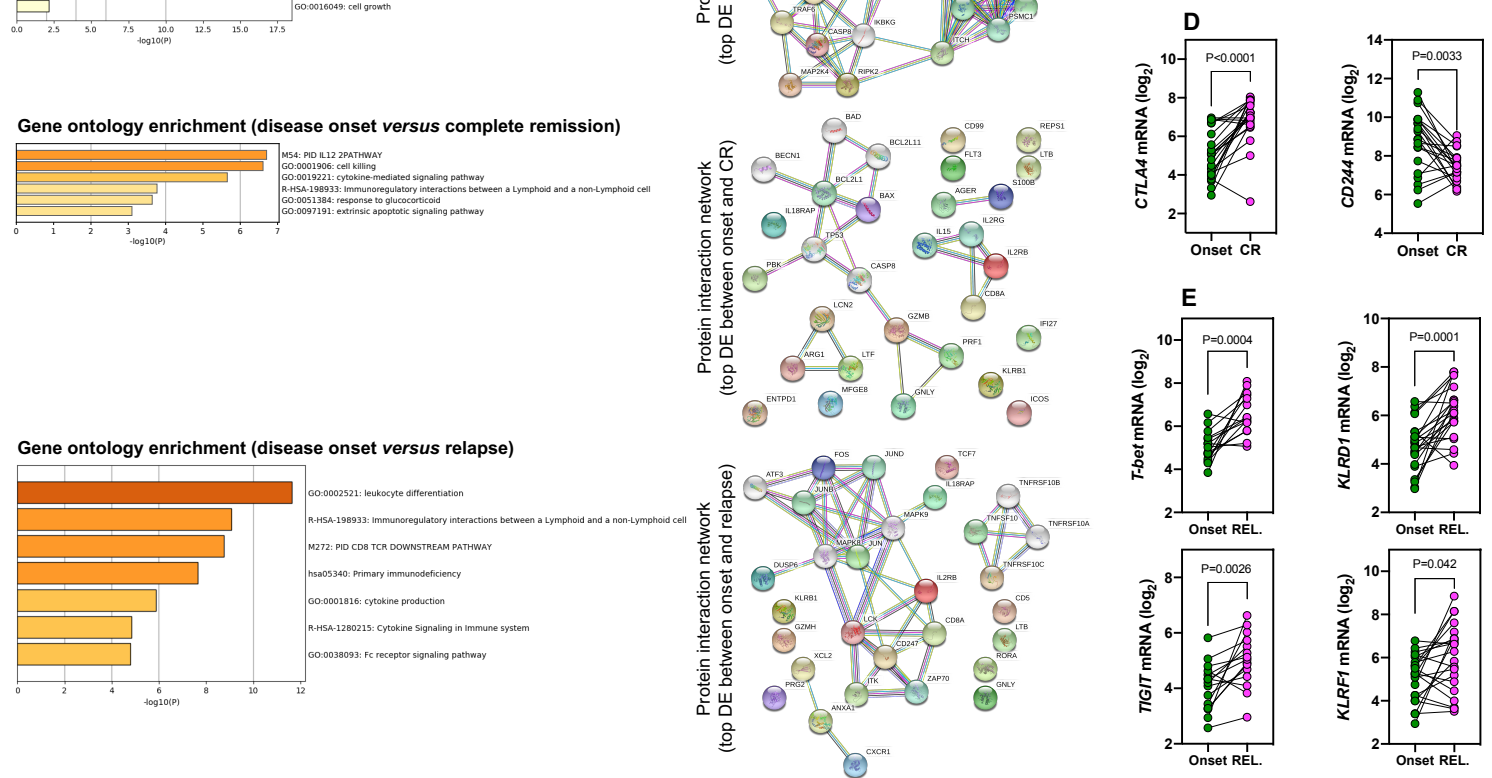

Fig. S10: Gene ontology and pathway analyses. A) Gene Ontology (GO) enrichment analysis. For each given gene list submitted to metascape.org, pathway and process enrichment analyses were carried out using all genes in the genome as the enrichment background. Terms with a $p$ value $<0.01$, a minimum count of 3 , and an enrichment factor $>1.5$ (defined as the ratio between the observed counts and the counts expected by chance) were collected and grouped into clusters based on their membership similarities. B) The Search Tool for the Retrieval of Interacting Genes/Proteins (STRING) database (http://stringdb.org) was used to critically assess and integrate protein-protein interactions between the top 20 genes differentially expressed in adults and children with AML, as well as across disease stages (diagnosis, achievement of complete remission [CR], relapse). A detailed list of significantly enriched GO processes and KEGG pathways ranked by false discovery rate $\left(<1 \times 10^{-7}\right)$ is provided as tables S4-S6. Protein network analysis and predicted functional partners of differentially expressed (DE) genes with the highest-confidence interaction scores (>0.900) are shown. Network nodes (query proteins) represent proteins produced by a single, protein-coding gene locus. White nodes represent second shells of interactors. Empty and filled nodes indicate proteins of unknown or partly known 3D structure, respectively. Edges represent protein-protein associations. Line shapes denote predicted modes of action. C) Tumor inflammation signature (TIS) and IFN- $\gamma$ scores across age groups. Data were compared using the Kruskal-Wallis test for unpaired determinations. D) Expression of select immune checkpoints and co-inhibitory molecules between matched BM samples from adult 
patients (SAL series) at disease onset and complete remission (CR; $n=22)$. E) Expression of genes associated with T-cell senescence/terminal differentiation/exhaustion (TIGIT, TBX21 or T-bet, KLRD1 and KLRF1) in matched BM samples from adult patients (SAL series) at disease onset and relapse $(n=22)$. Data were compared using the Mann-Whitney $U$ test for paired determinations. ${ }^{* *} \mathrm{P}<0.01$. ns $=$ not significant. 
A Immune cell type-specific scores
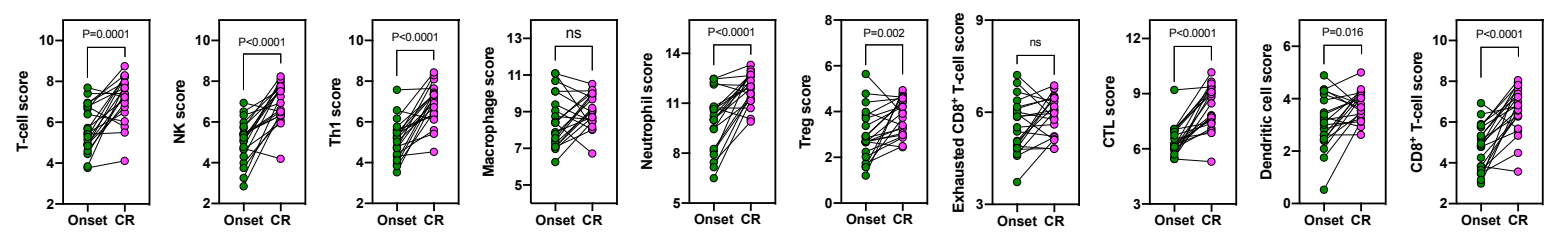

B Biological activity scores
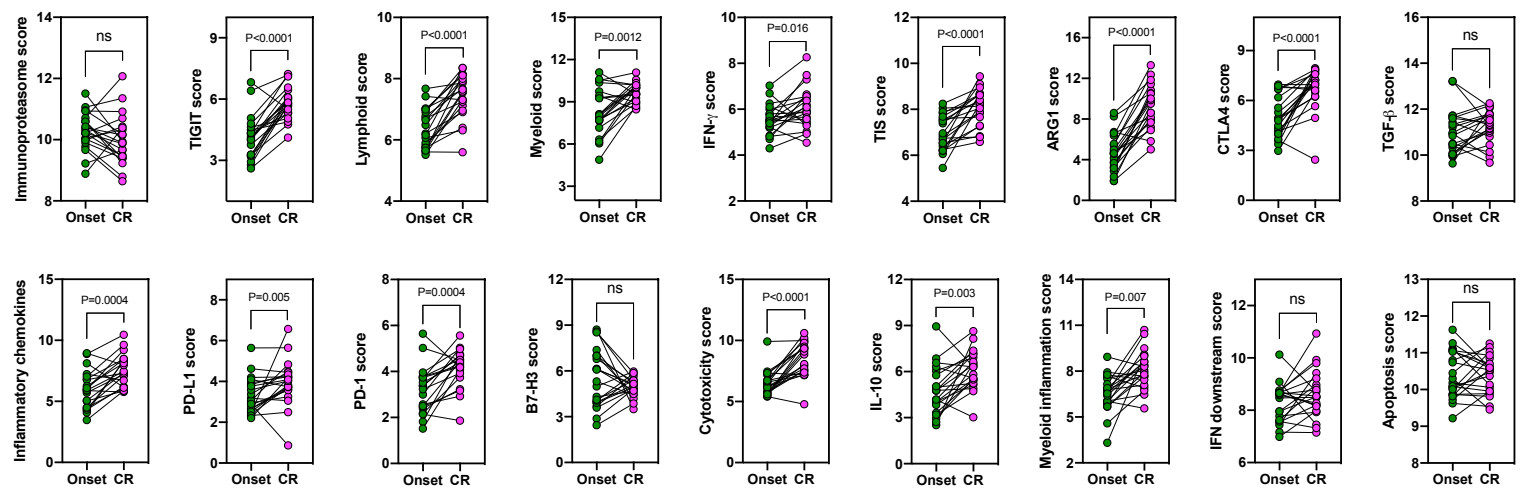

Fig. S11: Immune scores in AML patients (SAL cohort) at time of diagnosis and achievement of complete remission (paired samples). A) Immune cell type-specific scores in $\mathrm{BM}$ samples from 21 patients with $\mathrm{AML}$ at time of diagnosis and achievement of complete remission (CR; SAL cohort). B) Biological activity scores in BM samples from 21 patients with AML at time of diagnosis and achievement of complete remission (CR; SAL cohort). Data were compared using the Mann-Whitney $U$ test for paired determinations. 


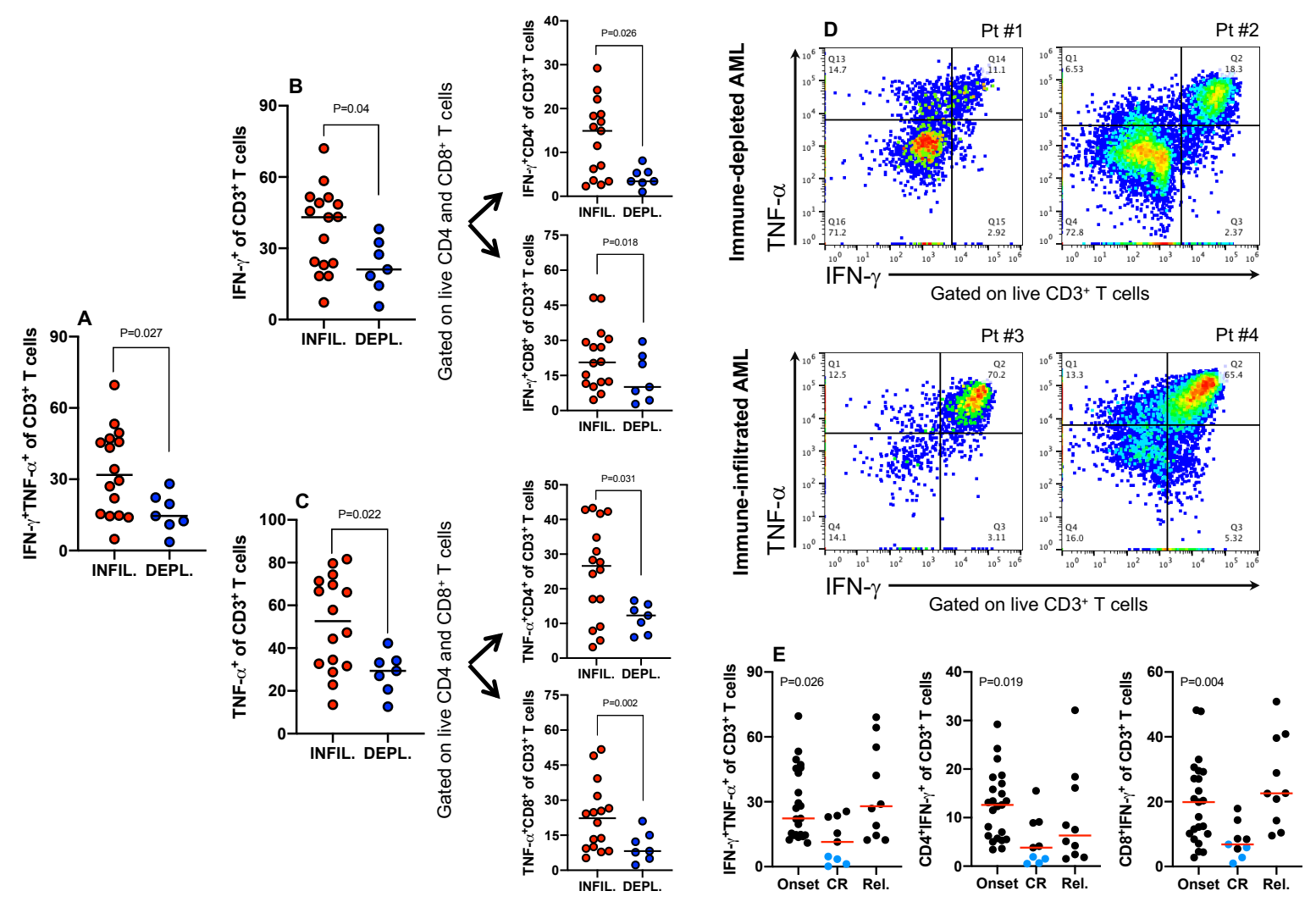

Fig. S12: Intracellular cytokine staining of BM samples from patients with immuneinfiltrated and immune-depleted AML. Frequency of polyfunctional IFN- $\gamma^{+} \mathrm{TNF}-\alpha^{+} \mathrm{T}$ cells (A), single-positive IFN- $\gamma^{+}$T cells (B) and single-positive TNF- $\alpha^{+}$T cells (C) in diagnostic BM samples from patients with immune-infiltrated $(n=16)$ and immune-depleted AML $(n=7)$. Comparisons were performed using the Mann-Whitney $U$ test for unpaired data. D) Flow cytometric analysis of intracellular cytokine production in representative BM samples from patients with immune-infiltrated (INFIL) and immune-depleted (DEPL) AML. E) Frequency of polyfunctional IFN $-\gamma^{+} \mathrm{TNF}-\alpha^{+} \mathrm{T}$ cells and IFN- $\gamma^{+}$-producing $\mathrm{CD} 4^{+}$and $\mathrm{CD} 8^{+} \mathrm{T}$ cells in diagnostic BM samples from AML patients at time of disease onset, achievement of complete remission (CR) and relapse. Data were compared using the Kruskal-Wallis test for unpaired determinations. Cases with documented minimal residual disease (MRD) negativity are shown as blue dots (immune-infiltrated subgroup). 
TCGA-AML series
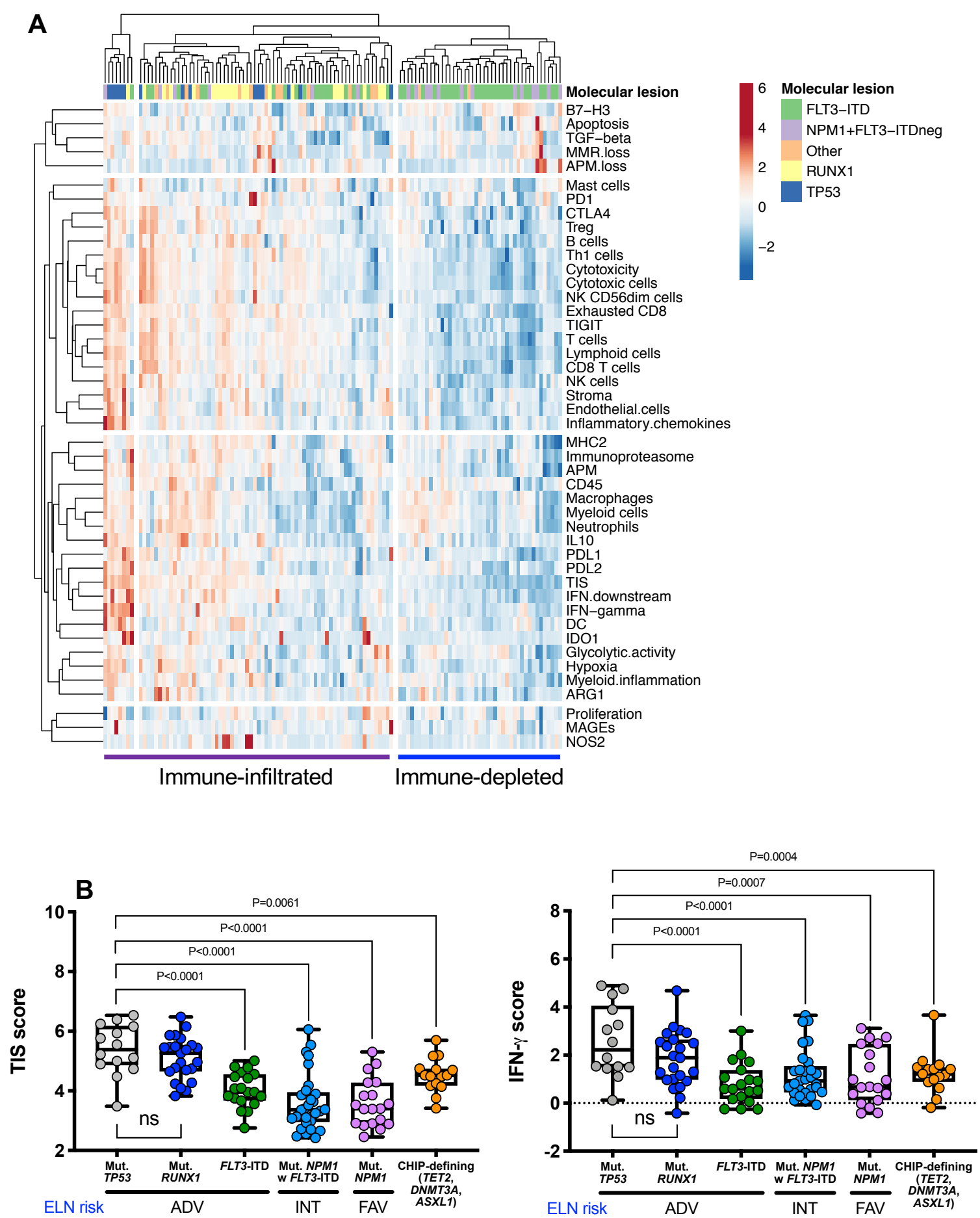

Fig. S13: Immune subtypes associate with cancer driver gene mutations in The Cancer Genome Atlas (TCGA)-AML specimens. A) Unsupervised hierarchical clustering (Euclidean distance, complete linkage) of immune cell type signatures and biological activity signatures in TCGA-AML cases stratified by prognostic molecular lesions. ClustVis was used for data analysis and visualization. B) Expression of IFN-related gene sets (as defined in Fig. 1) in TCGA cases with TP53 mutations (adverse molecular risk), NPM1 mutations without FLT3-ITD (ELN favorable 
risk), FLT3-ITD without NPM1 mutations (ELN adverse risk), RUNX1 mutations (adverse molecular risk). Other = clonal hematopoiesis of indeterminate potential (CHIP)-defining mutations (TET2, DNMT3A, ASXL1). Comparisons were performed using the Kruskal-Wallis test for unpaired determinations. 


\section{Beat-AML series}
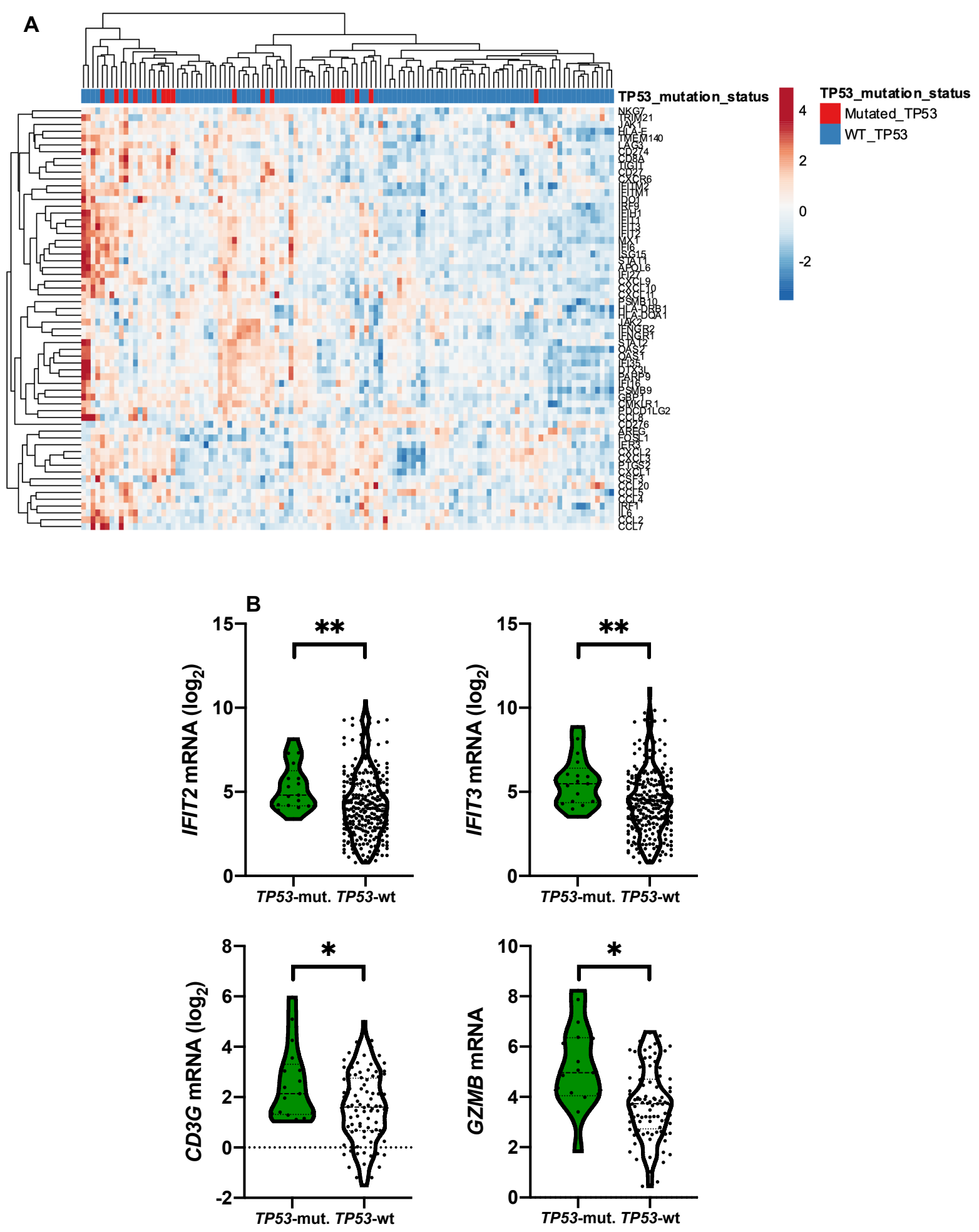

Fig. S14: Genes implicated in IFN downstream signaling in Beat AML trial specimens with TP53 mutations. A) Expression of IFN-downstream genes in Beat AML cases with $(n=21)$ or without TP53 mutations $(n=128)$. ClustVis was used for data analysis and visualization. B) Violin plots summarizing the expression of surrogate markers of T-cell infiltration and cytotoxicity in Beat 
AML cases with or without TP53 mutations. Comparisons were performed with the Mann-Whitney $U$ test for unpaired data. ${ }^{*} P<0.05$; ${ }^{*} P<0.01$. 

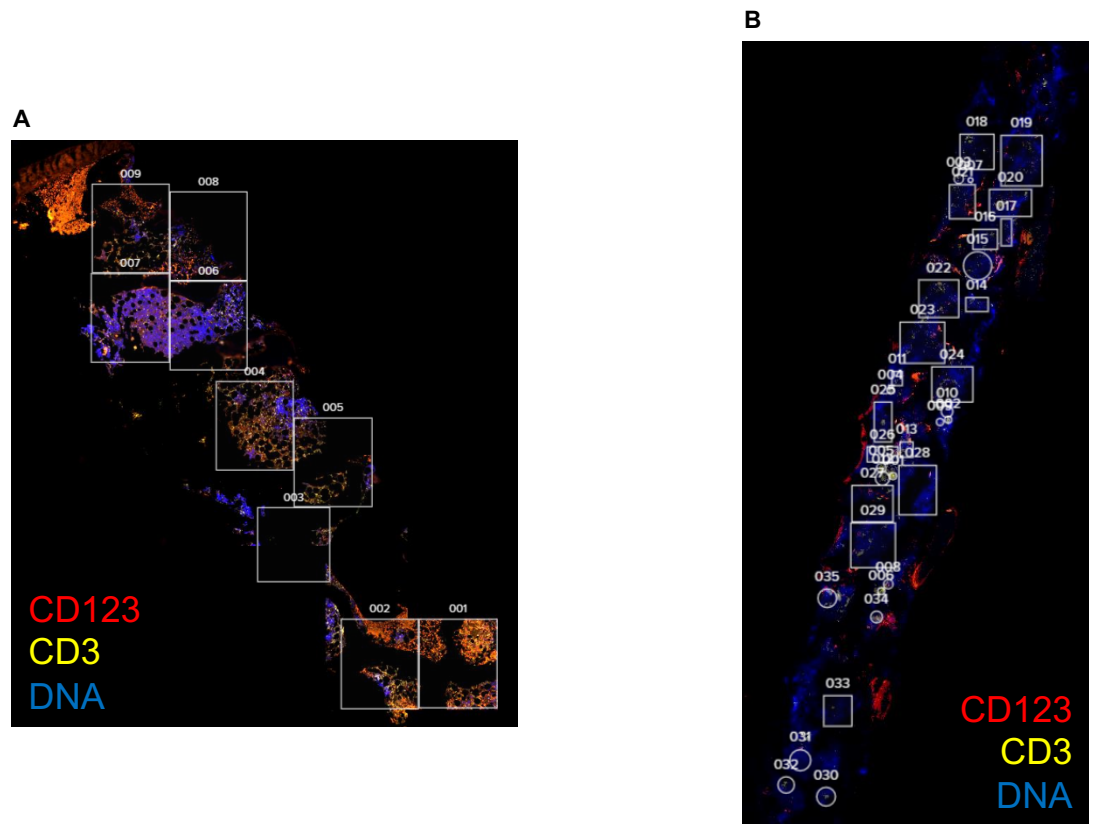

Fig. S15: Identification of regions of interest in BM biopsies from patients receiving flotetuzumab immunotherapy. Regions of interest (ROIs) covering the entire FFPE BM biopsy were selected for highly multiplexed protein profiling on the GeoMx DSP platform. Two representative patients are shown in panels $A$ and $B$. 


\section{Supplementary Tables}

Supplemental Table 1. Differentially expressed (DE) genes (false discovery rate<0.05) between ELN favorable-risk and adverse-risk AML (HOVON discovery cohort).

\begin{tabular}{|c|c|c|c|c|}
\hline $\begin{array}{l}\text { Official } \\
\text { symbol }\end{array}$ & Official full name & Location & NanoString probe ID & $\begin{array}{l}\text { Synonyms; previous } \\
\text { symbols }\end{array}$ \\
\hline$A P P$ & $\begin{array}{l}\text { Amyloid beta } \\
\text { precursor protein }\end{array}$ & $21 q 21.3$ & NM_000484.3:1725 & AD1 \\
\hline ARG2 & Arginase 2 & $14 q 24.1$ & NM_001172.3:1150 & \\
\hline CARD11 & $\begin{array}{l}\text { Caspase } \\
\text { recruitment domain } \\
\text { family member } 11\end{array}$ & $7 p 22.2$ & NM_032415.2:1075 & CARMA1, BIMP3 \\
\hline CCL23 & $\begin{array}{lr}\text { C-C } & \text { motif } \\
\text { chemokine } & \text { ligand } \\
23 & \end{array}$ & $17 q 12$ & NM_145898.1:336 & $\begin{array}{lr}\text { SCYA23, } & \text { Ckb-8, } \\
\text { MPIF-1, MIP-3, CKb8 }\end{array}$ \\
\hline CD34 & CD34 molecule & $1 q 32.2$ & NM_001025109.1:1580 & \\
\hline CD55 & CD55 molecule & $1 q 32.2$ & NM_000574.3:101 & DAF, CR, TC, CROM \\
\hline CT45A1 & $\begin{array}{l}\text { Cancer/testis } \\
\text { antigen family } 45 \\
\text { member A1 }\end{array}$ & Xq26.3 & NM_001017417.1:866 & CT45-1, CT45.1 \\
\hline CYFIP2 & $\begin{array}{l}\text { Cytoplasmic FMR1 } \\
\text { interacting protein } 2\end{array}$ & $5 q 33.3$ & NM_001037332.2:4043 & PIR121 \\
\hline DPP4 & $\begin{array}{l}\text { Dipeptidyl } \\
\text { peptidase } 4\end{array}$ & $2 q 24.2$ & NM_001935.3:2700 & $\begin{array}{ll}\text { CD26, } & \text { ADCP2, } \\
\text { DPPIV } & \end{array}$ \\
\hline F2RL1 & $\begin{array}{l}\text { F2R like trypsin } \\
\text { receptor } 1\end{array}$ & $5 q 13.3$ & NM_005242.3:940 & GPR11, PAR2 \\
\hline IL15 & Interleukin 15 & $4 q 31.21$ & NM_172174.1:1685 & IL-15, MGC9721 \\
\hline IL2RA & $\begin{array}{lr}\text { Interleukin } & 2 \\
\text { receptor } & \text { subunit } \\
\text { alpha } & \end{array}$ & $10 \mathrm{p} 15.1$ & NM_000417.1:1000 & IL2R, IDDM10, CD25 \\
\hline LILRA4 & $\begin{array}{l}\text { Leukocyte } \\
\text { immunoglobulin like } \\
\text { receptor A4 }\end{array}$ & $19 q 13.42$ & NM_012276.3:1577 & \\
\hline MAF & $\begin{array}{l}\text { MAF bZIP } \\
\text { transcription factor }\end{array}$ & $16 q 23.2$ & NM_005360.4:888 & c-MAF \\
\hline MRC1 & $\begin{array}{l}\text { Mannose receptor } \\
\text { C-type } 1\end{array}$ & 10p12.33 & NM_002438.2:525 & $\begin{array}{l}\text { MRC1L1, CLEC13D, } \\
\text { CD206, bA541I19.1, } \\
\text { CLEC13DL }\end{array}$ \\
\hline NFATC1 & $\begin{array}{l}\text { Nuclear factor of } \\
\text { activated T cells } 1\end{array}$ & $18 q 23$ & NM_172389.1:1984 & $\begin{array}{l}\text { NF-ATC, NFATc, } \\
\text { NFAT2 }\end{array}$ \\
\hline PNMA1 & $\begin{array}{l}\text { PNMA family } \\
\text { member } 1\end{array}$ & $14 q 24.3$ & NM_006029.4:1565 & MA1 \\
\hline PRM1 & Protamine 1 & $16 p 13.13$ & NM_002761.2:319 & CT94.1 \\
\hline RAG1 & $\begin{array}{l}\text { Recombination } \\
\text { activating } 1\end{array}$ & $11 \mathrm{p} 12$ & NM_000448.2:2300 & RNF74, MGC43321 \\
\hline STAT4 & $\begin{array}{l}\text { Signal transducer } \\
\text { and activator of } \\
\text { transcription } 4\end{array}$ & $\begin{array}{l}2 q 32.2- \\
q 32.3\end{array}$ & NM_003151.2:789 & ILT7, CD85g \\
\hline
\end{tabular}




\begin{tabular}{|l|l|l|l|l|}
\hline TNFRSF18 & $\begin{array}{l}\text { TNF receptor } \\
\text { superfamily } \\
\text { member 18 }\end{array}$ & $1 \mathrm{p} 36.33$ & NM_004195.2:445 & AITR, GITR, CD357 \\
\hline
\end{tabular}


Table S2. Competing risks analysis for overall survival (OS) prediction (PMCC discovery cohort).

\begin{tabular}{|c|c|c|c|c|c|}
\hline & SHR & Std. Err. & $\mathrm{z}$ & $\mathrm{P}>/ \mathrm{z} /$ & $95 \% \mathrm{Cl}$ \\
\hline IFN score & 1.053864 & 0.0266407 & 2.08 & $\mathbf{0 . 0 3 8}$ & $\begin{array}{c}1.002922- \\
1.107394\end{array}$ \\
\hline Innate score & 0.9683486 & 0.0264452 & -1.18 & 0.239 & $\begin{array}{c}0.9178796- \\
1.021593\end{array}$ \\
\hline $\begin{array}{c}\text { Adaptive } \\
\text { score }\end{array}$ & 0.9996771 & 0.0090025 & -0.04 & 0.971 & $\begin{array}{c}0.9821873- \\
1.017478\end{array}$ \\
\hline
\end{tabular}

Number of observations $=249$

Number failed $=125$

Number competing $=75$

Number censored $=49$

Wald $\chi^{2}=6.15$

Legend: SHR = Estimated subhazard ratio; Std. Err. = standard error; $\mathrm{Cl}=$ Confidence interval; IFN = interferon.

Competing risk analyses were performed using STATA/IC (version 16.0).

HSCT, a potential confounder, was treated as an event whose occurrence precluded the occurrence of the primary clinical endpoint (death). 
Table S3. Causes of death in patients receiving allogeneic hematopoietic stem cell transplantation (HSCT; PMCC cohort).

\begin{tabular}{|l|c|c|}
\hline Cause of death & Immune-infiltrated $(\mathbf{n = 3 6 )}$ & Immune-depleted (n= 52) \\
\hline Treatment-related toxicity* & $3(8 \%)$ & $1(2 \%)$ \\
\hline Graft-versus-host disease & $8(22 \%)$ & $8(15 \%)$ \\
\hline Leukemia recurrence & $9(25 \%)$ & $11(21 \%)$ \\
\hline Infections & $3(8 \%)$ & $9(17 \%)$ \\
\hline Other; not known & $0 ; 13(36 \%)$ & $4 ; 19(44 \%)$ \\
\hline
\end{tabular}

*Including sinusoidal obstruction syndrome. 
Table S4. Gene ontologies (GO) of and KEGG pathways captured by DE genes between adult (SAL cohort) and childhood AML (CHOP cohort).

\begin{tabular}{|c|c|c|c|}
\hline GO term & Description & $\begin{array}{l}\text { Count in } \\
\text { gene set }\end{array}$ & FDR \\
\hline GO:0002376 & Immune system process & 26 & $4.03 \times 10^{-17}$ \\
\hline GO:0006955 & Immune response & 22 & $1.79 \times 10^{-15}$ \\
\hline GO:0016579 & Protein deubiquitination & 13 & $5.31 \times 10^{-14}$ \\
\hline GO:0071347 & Cellular response to interleukin 1 & 10 & $1.24 \times 10^{-12}$ \\
\hline GO:0070423 & $\begin{array}{l}\text { Nucleotide-binding oligomerization domain } \\
\text { containing signaling pathway }\end{array}$ & 7 & $1.01 \times 10^{-11}$ \\
\hline GO:0006508 & Proteolysis & 17 & $2.30 \times 10^{-11}$ \\
\hline GO:0045321 & Leukocyte activation & 15 & $9.49 \times 10^{-11}$ \\
\hline GO:0006950 & Response to stress & 23 & $1.03 \times 10^{-10}$ \\
\hline GO:0050896 & Response to stimulus & 30 & $1.33 \times 10^{-10}$ \\
\hline GO:0070498 & Interleukin 1-mediated signaling pathway & 7 & $2.76 \times 10^{-10}$ \\
\hline GO:0006954 & Inflammatory response & 12 & $3.22 \times 10^{-10}$ \\
\hline GO:0002758 & $\begin{array}{l}\text { Innate immune response-activating signal } \\
\text { transduction }\end{array}$ & 9 & $3.62 \times 10^{-10}$ \\
\hline GO:0002221 & Pattern recognition receptor signaling pathway & 8 & $7.58 \times 10^{-10}$ \\
\hline GO:0051603 & $\begin{array}{l}\text { Proteolysis involved in cellular protein catabolic } \\
\text { process }\end{array}$ & 12 & $7.58 \times 10^{-10}$ \\
\hline GO:0070647 & $\begin{array}{l}\text { Protein modification by small protein } \\
\text { conjugation or removal }\end{array}$ & 14 & $1.91 \times 10^{-9}$ \\
\hline GO:0006511 & Ubiquitin-dependent protein catabolic process & 11 & $3.24 \times 10^{-9}$ \\
\hline GO:0034097 & Response to cytokine & 14 & $5.12 \times 10^{-9}$ \\
\hline GO:0010033 & Response to organic substance & 20 & $5.49 \times 10^{-9}$ \\
\hline GO:0019221 & Cytokine-mediated signaling pathway & 12 & $6.08 \times 10^{-9}$ \\
\hline Pathway ID & Description & $\begin{array}{l}\text { Count in } \\
\text { gene set }\end{array}$ & FDR \\
\hline hsa05169 & Epstein-Barr virus infection & 17 & $1.92 \times 10^{-24}$ \\
\hline hsa03050 & Proteasome & 10 & $1.21 \times 10^{-17}$ \\
\hline hsa04620 & Toll-like receptor signaling pathway & 10 & $2.03 \times 10^{-14}$ \\
\hline hsa05142 & Chagas disease (American trypanosomiasis) & 10 & $2.03 \times 10^{-14}$ \\
\hline hsa04657 & IL-17 signaling pathway & 9 & $4.62 \times 10^{-13}$ \\
\hline hsa04621 & NOD-like receptor signaling pathway & 10 & $1.12 \times 10^{-12}$ \\
\hline hsa04668 & TNF signaling pathway & 9 & $1.28 \times 10^{-12}$ \\
\hline hsa04062 & Chemokine signaling pathway & 10 & $1.92 \times 10^{-12}$ \\
\hline hsa05167 & $\begin{array}{l}\text { Kaposi's sarcoma-associated herpesvirus } \\
\text { infection }\end{array}$ & 10 & $1.92 \times 10^{-12}$ \\
\hline hsa04064 & NF-kappa B signaling pathway & 8 & $1.98 \times 10^{-11}$ \\
\hline hsa04622 & RIG-I-like receptor signaling pathway & 7 & $1.86 \times 10^{-10}$ \\
\hline hsa05161 & Hepatitis B & 8 & $4.09 \times 10^{-10}$ \\
\hline hsa05134 & Legionellosis & 6 & $2.72 \times 10^{-9}$ \\
\hline hsa05131 & Shigellosis & 6 & $5.99 \times 10^{-9}$ \\
\hline hsa05120 & $\begin{array}{l}\text { Epithelial cell signaling in Helicobacter pylori } \\
\text { infection }\end{array}$ & 6 & $7.27 \times 10^{-9}$ \\
\hline hsa05132 & Salmonella infection & 6 & $2.66 \times 10^{-8}$ \\
\hline
\end{tabular}




\begin{tabular}{|l|l|l|l|}
\hline hsa05168 & Herpes simplex infection & 7 & $6.58 \times 10^{-8}$ \\
\hline hsa05145 & Toxoplasmosis & 6 & $1.04 \times 10^{-7}$ \\
\hline hsa04380 & Osteoclast differentiation & 6 & $2.06 \times 10^{-7}$ \\
\hline hsa05160 & Hepatitis C & 6 & $2.68 \times 10^{-7}$ \\
\hline
\end{tabular}


Table S5. GO of and KEGG pathways captured by DE genes between adult AML patients at the time of diagnosis and achievement of complete remission (SAL cohort).

\begin{tabular}{|c|c|c|c|}
\hline GO term & Description & $\begin{array}{l}\text { Count in } \\
\text { gene set }\end{array}$ & FDR \\
\hline GO:0002376 & Immune system process & 20 & $7.85 \times 10^{-9}$ \\
\hline GO:0046902 & $\begin{array}{l}\text { Regulation of mitochondrial membrane } \\
\text { permeability }\end{array}$ & 7 & $2.04 \times 10^{-8}$ \\
\hline GO:0052150 & $\begin{array}{l}\text { Modulation by symbiont of host apoptotic } \\
\text { process }\end{array}$ & 4 & $2.49 \times 10^{-7}$ \\
\hline GO:1902533 & $\begin{array}{l}\text { Positive regulation of intracellular signal } \\
\text { transduction }\end{array}$ & 13 & $2.49 \times 10^{-7}$ \\
\hline GO:0006955 & Immune response & 15 & $2.58 \times 10^{-7}$ \\
\hline GO:0007166 & Cell surface receptor signaling pathway & 17 & $2.58 \times 10^{-7}$ \\
\hline GO:0019221 & Cytokine-mediated signaling pathway & 11 & $2.58 \times 10^{-7}$ \\
\hline GO:0031341 & Regulation of cell killing & 6 & $2.58 \times 10^{-7}$ \\
\hline GO:0034097 & Response to cytokine & 13 & $2.58 \times 10^{-7}$ \\
\hline GO:0071310 & Cellular response to organic substance & 17 & $2.60 \times 10^{-7}$ \\
\hline GO:0010821 & Regulation of mitochondrion organization & 7 & $2.95 \times 10^{-7}$ \\
\hline GO:1901030 & $\begin{array}{l}\text { Positive regulation of mitochondrial outer } \\
\text { membrane permeabilization involved in } \\
\text { apoptotic signaling pathway }\end{array}$ & 5 & $3.11 \times 10^{-7}$ \\
\hline GO:0048583 & Regulation of response to stimulus & 21 & $3.59 \times 10^{-7}$ \\
\hline GO:0070887 & Cellular response to chemical stimulus & 18 & $4.02 \times 10^{-7}$ \\
\hline GO:0044419 & Interspecies interaction between organisms & 11 & $4.46 \times 10^{-7}$ \\
\hline GO:0010822 & $\begin{array}{l}\text { Positive regulation of mitochondrion } \\
\text { organization }\end{array}$ & 6 & $4.67 \times 10^{-7}$ \\
\hline GO:0071345 & Cellular response to cytokine stimulus & 12 & $5.40 \times 10^{-7}$ \\
\hline GO:0048584 & Positive regulation of response to stimulus & 16 & $5.56 \times 10^{-7}$ \\
\hline GO:2001235 & $\begin{array}{l}\text { Positive regulation of apoptotic signaling } \\
\text { pathway }\end{array}$ & 7 & $5.56 \times 10^{-7}$ \\
\hline Pathway ID & Description & $\begin{array}{l}\text { Count in } \\
\text { gene set }\end{array}$ & FDR \\
\hline hsa04210 & Apoptosis & 8 & $3.58 \times 10^{-9}$ \\
\hline hsa04215 & Apoptosis - multiple species & 5 & $1.14 \times 10^{-7}$ \\
\hline hsa05200 & Pathways in cancer & 10 & $1.15 \times 10^{-7}$ \\
\hline hsa01524 & Platinum drug resistance & 5 & $2.51 \times 10^{-6}$ \\
\hline hsa05202 & Transcriptional mis-regulation in cancer & 6 & $4.90 \times 10^{-6}$ \\
\hline hsa04151 & PI3K-Akt signaling pathway & 7 & $1.52 \times 10^{-5}$ \\
\hline hsa05014 & Amyotrophic lateral sclerosis (ALS) & 4 & $2.03 \times 10^{-5}$ \\
\hline hsa05166 & HTLV-I infection & 6 & $2.84 \times 10^{-5}$ \\
\hline hsa04060 & Cytokine-cytokine receptor interaction & 6 & $3.36 \times 10^{-5}$ \\
\hline hsa05212 & Pancreatic cancer & 4 & $6.24 \times 10^{-5}$ \\
\hline hsa05220 & Chronic myeloid leukemia & 4 & $6.27 \times 10^{-5}$ \\
\hline hsa01521 & EGFR tyrosine kinase inhibitor resistance & 4 & $6.35 \times 10^{-5}$ \\
\hline hsa05210 & Colorectal cancer & 4 & $8.12 \times 10^{-5}$ \\
\hline hsa05340 & Primary immunodeficiency & 3 & 0.00023 \\
\hline hsa05161 & Hepatitis B & 4 & 0.00049 \\
\hline
\end{tabular}




\begin{tabular}{|l|l|l|l|}
\hline hsa04630 & JAK-STAT signaling pathway & 4 & 0.00072 \\
\hline hsa05213 & Endometrial cancer & 3 & 0.00072 \\
\hline hsa05225 & Hepatocellular carcinoma & 4 & 0.00072 \\
\hline hsa04137 & Mitophagy - animal & 3 & 0.00077 \\
\hline hsa05223 & Non-small cell lung cancer & 3 & 0.00084 \\
\hline
\end{tabular}


Table S6. GO of and KEGG pathways captured by DE genes between adult AML patients at the time of relapse and achievement of complete remission (SAL cohort).

\begin{tabular}{|c|c|c|c|}
\hline GO term & Description & $\begin{array}{l}\text { Count in } \\
\text { gene set }\end{array}$ & FDR \\
\hline GO:0007166 & Cell surface receptor signaling pathway & 23 & $1.96 \times 10^{-13}$ \\
\hline GO:0002376 & Immune system process & 21 & $1.90 \times 10^{-10}$ \\
\hline GO:0034097 & Response to cytokine & 16 & $1.90 \times 10^{-10}$ \\
\hline GO:0006955 & Immune response & 18 & $2.07 \times 10^{-10}$ \\
\hline GO:0007165 & Signal transduction & 26 & $2.56 \times 10^{-10}$ \\
\hline GO:0051716 & Cellular response to stimulus & 28 & $5.39 \times 10^{-10}$ \\
\hline GO:0071345 & Cellular response to cytokine stimulus & 14 & $4.73 \times 10^{-9}$ \\
\hline GO:0050896 & Response to stimulus & 29 & $7.20 \times 10^{-9}$ \\
\hline GO:0010033 & Response to organic substance & 20 & $1.27 \times 10^{-9}$ \\
\hline GO:0019221 & Cytokine-mediated signaling pathway & 12 & $1.27 \times 10^{-9}$ \\
\hline GO:0071310 & Cellular response to organic substance & 18 & $2.31 \times 10^{-9}$ \\
\hline GO:0010941 & Regulation of cell death & 16 & $2.72 \times 10^{-9}$ \\
\hline GO:0002521 & Leukocyte differentiation & 9 & $7.30 \times 10^{-9}$ \\
\hline GO:0009612 & Response to mechanical stimulus & 8 & $8.11 \times 10^{-9}$ \\
\hline GO:0030217 & T cell differentiation & 7 & $1.02 \times 10^{-7}$ \\
\hline GO:0010942 & Positive regulation of cell death & 11 & $1.63 \times 10^{-7}$ \\
\hline GO:0050776 & Regulation of immune response & 12 & $1.90 \times 10^{-7}$ \\
\hline GO:0002682 & Regulation of immune system process & 14 & $2.88 \times 10^{-7}$ \\
\hline GO:0002768 & $\begin{array}{l}\text { Immune response-regulating cell surface } \\
\text { receptor signaling pathway }\end{array}$ & 8 & $3.57 \times 10^{-7}$ \\
\hline Pathway ID & Description & $\begin{array}{l}\text { Count in } \\
\text { gene set }\end{array}$ & FDR \\
\hline hsa04659 & Th17 cell differentiation & 9 & $5.81 \times 10^{-12}$ \\
\hline hsa04658 & Th1 and Th2 cell differentiation & 8 & $6.86 \times 10^{-11}$ \\
\hline hsa04660 & T cell receptor signaling pathway & 8 & $1.11 \times 10^{-10}$ \\
\hline hsa04210 & Apoptosis & 8 & $8.77 \times 10^{-10}$ \\
\hline hsa04060 & Cytokine-cytokine receptor interaction & 9 & $3.86 \times 10^{-9}$ \\
\hline hsa04380 & Osteoclast differentiation & 7 & $1.58 \times 10^{-8}$ \\
\hline hsa04650 & Natural killer cell mediated cytotoxicity & 7 & $1.58 \times 10^{-8}$ \\
\hline hsa05164 & Influenza A & 7 & $8.94 \times 10^{-8}$ \\
\hline hsa04217 & Necroptosis & 6 & $1.63 \times 10^{-6}$ \\
\hline hsa05210 & Colorectal cancer & 5 & $2.47 \times 10^{-6}$ \\
\hline hsa04657 & IL-17 signaling pathway & 5 & $3.27 \times 10^{-6}$ \\
\hline hsa05142 & Chagas disease (American trypanosomiasis) & 5 & $4.67 \times 10^{-6}$ \\
\hline hsa04668 & TNF signaling pathway & 5 & $5.93 \times 10^{-6}$ \\
\hline hsa05162 & Measles & 5 & $1.48 \times 10^{-5}$ \\
\hline hsa05166 & HTLV-I infection & 6 & $1.48 \times 10^{-5}$ \\
\hline hsa05120 & $\begin{array}{l}\text { Epithelial cell signaling in Helicobacter pylori } \\
\text { infection }\end{array}$ & 4 & $2.48 \times 10^{-5}$ \\
\hline hsa04010 & MAPK signaling pathway & 6 & $3.20 \times 10^{-5}$ \\
\hline hsa05133 & Pertussis & 4 & $3.40 \times 10^{-5}$ \\
\hline hsa05132 & Salmonella infection & 4 & $5.21 \times 10^{-5}$ \\
\hline hsa01522 & Endocrine resistance & 4 & $7.90 \times 10^{-5}$ \\
\hline
\end{tabular}


Table S7: Binary logistic regression predicting therapeutic resistance (PMCC cohort).

Model 1

ELN risk category

Favorable vs. adverse

Intermediate vs. adverse

Model $\chi 2$

$P$ value

$-2 L L$

Nagelkerke (pseudo R2)

Sensitivity

Specificity

False positive rate

False negative rate

AUROC

\section{Model 2}

ELN risk category

Favorable vs. adverse

Intermediate vs. adverse

WBC

Model $\chi 2$

$P$ value

$-2 \mathrm{LL}$

Nagelkerke (pseudo R2)

Sensitivity

Specificity

False positive rate

False negative rate

AUROC

\section{Model 3}

ELN risk category

Favorable vs. adverse

Intermediate vs. adverse

WBC

AML type (primary versus secondary)

Model $\chi 2$

$P$ value

$-2 L L$

Nagelkerke (pseudo R2)

Sensitivity

Specificity

False positive rate

False negative rate

AUROC
B

$-2.97$

$-1.52$

33.43

0

254.6

0.183

$48.0 \%$

$85.2 \%$

$45.8 \%$

$17.9 \%$

0.702

\section{B}

$-3.001$

$-1.613$

0.003

34.82

0

253.2

0.190

$50.0 \%$

$85.2 \%$

$45.0 \%$

$17.5 \%$

0.731

B

$-3.005$

$-1.583$

0.004

$-0.65$

37.33

0

250.6

0.203

$50.0 \%$

$85.2 \%$

$45.0 \%$

$17.5 \%$

0.731
Wald $\chi 2 \quad p \quad$ OR

28.64

$\begin{array}{lll}14.77 & 0 & 0.05\end{array}$

$\begin{array}{lll}21.36 & 0 & 0.22\end{array}$

$\begin{array}{lll}\text { Wald } \chi 2 & \boldsymbol{p} & \text { OR } \\ 29.63 & 0 & \\ 15.1 & 0 & 0.05 \\ 22.37 & 0 & 0.2 \\ 1.44 & 0.23 & 1.003\end{array}$

Model 4

B

Wald $\chi^{2} \quad p \quad$ OR

Wald $\chi^{2} \quad p \quad$ OR

28.51

$\begin{array}{lll}14.88 & 0 & 0.05\end{array}$

$\begin{array}{lll}21.29 & 0 & 0.22\end{array}$

1.84

$0.175 \quad 1.004$

2.59

$0.108 \quad 0.52$ 
ELN risk category

Favorable vs. adverse

Intermediate vs. adverse

WBC

AML type (primary versus secondary)

Age

Model $\chi 2$

$P$ value

$-2 L L$

Nagelkerke (pseudo R2)

Sensitivity

Specificity

False positive rate

False negative rate

AUROC

\section{Model 5}

ELN risk category

Favorable vs. adverse

Intermediate vs. adverse

WBC

AML type (primary versus secondary)

Age

Myeloid inflammation

Inflammatory chemokines

IFN- $\gamma$

IFN downstream

Immunoproteasome

IL-10

PD-L1

PD-L2

MAGEs

Model $\chi 2$

$\mathrm{P}$ value

$-2 L L$

Nagelkerke (pseudo R2)

Sensitivity

Specificity

False positive rate

False negative rate

AUROC
$28.35 \quad 0$

$\begin{array}{lll}15.37 & 0 & 0.05\end{array}$

$\begin{array}{lll}20.57 & 0 & 0.21\end{array}$

$\begin{array}{lll}1.54 & 0.215 & 1.003\end{array}$

$\begin{array}{lll}3.371 & 0.066 & 0.457\end{array}$

$\begin{array}{lll}1.111 & 0.292 & 0.989\end{array}$
38.45

0

249.5

0.318

$50.0 \%$

$85.2 \%$

$46.0 \%$

$17.5 \%$

0.775

B

$-3.63$

$-1.65$

0.006

$-0.49$

$-0.02$

0.6

$-0.37$

$-0.82$

0.87

0.62

$-0.17$

$-0.09$

0.397

0.16

65.87

0

222.1

0.339

$63.6 \%$

$88.0 \%$

$34.3 \%$

$13.0 \%$

0.815
Wald $\chi 2 \quad p \quad$ OR

27.38

$\begin{array}{lll}18.83 & 0 & 0.03\end{array}$

$\begin{array}{lll}16.9 & 0 & 6\end{array}$

$\begin{array}{lll}3.83 & \mathbf{0 . 0 5} & 1.003\end{array}$

$\begin{array}{lll}0.84 & 0.36 & 0.65\end{array}$

$\begin{array}{lll}1.36 & 0.244 & 0.985\end{array}$

$\begin{array}{lll}9.06 & \mathbf{0 . 0 0 3} & 1.822\end{array}$

$\begin{array}{lll}1.99 & 0.16 & 0.69\end{array}$

$\begin{array}{lll}6.07 & \mathbf{0 . 0 1 4} & 0.439\end{array}$

$\begin{array}{lll}4.49 & \mathbf{0 . 0 3 4} 2.4\end{array}$

$\begin{array}{lll}1.29 & 0.256 & 1.86\end{array}$

$\begin{array}{lll}0.58 & 0.45 & 0.85\end{array}$

$\begin{array}{lll}0.58 & 0.75 & 0.85 \\ 0.07 & 0.79 & 0.914\end{array}$

$\begin{array}{lll}0.92 & 0.166 & 1.49\end{array}$

$\begin{array}{lll}0.11 & 0.74 & 1.18\end{array}$

Legend: Predicted likelihood of response to induction chemotherapy in AML patients from the PMCC cohort ( $n=249$ cases). 2017 ELN cytogenetic risk, white blood cell count at diagnosis, disease type (primary versus secondary $\mathrm{AML}$ ), patient age at diagnosis and immune gene signatures were selected as pre-treatment covariates. 
Table S8: Multinomial logistic regression predicting therapeutic response (HOVON cohort).

Parameter estimates

\begin{tabular}{|c|c|c|c|c|c|c|c|c|}
\hline \multirow[b]{2}{*}{ EFS } & \multirow[b]{2}{*}{ Intorcent } & $\beta$ & SE & Wald $\chi^{2}$ & \multirow{2}{*}{$\begin{array}{l}\text { P value } \\
0.69\end{array}$} & \multirow[t]{2}{*}{ HR } & \multicolumn{2}{|c|}{$95 \% \mathrm{Cl}$ for $\mathrm{HR}$} \\
\hline & & -2.345 & 5.881 & 0.159 & & & & \\
\hline & BM blasts & 0 & 0.006 & 0.002 & 0.966 & 1 & 0.988 & 1.011 \\
\hline & Age & -0.006 & 0.009 & 0.498 & 0.48 & 0.994 & 0.977 & 1.011 \\
\hline & Lymphoid cells & 1.487 & 1.48 & 1.01 & 0.315 & 4.425 & 0.243 & 80.533 \\
\hline & Myeloid cells & -0.309 & 0.441 & 0.492 & 0.483 & 0.734 & 0.309 & 1.742 \\
\hline & APM & -0.148 & 0.585 & 0.064 & 0.801 & 0.863 & 0.274 & 2.717 \\
\hline & MHC2 & -0.24 & 0.132 & 3.323 & 0.068 & 0.787 & 0.608 & 1.018 \\
\hline & IFN- $\gamma$ & 0.271 & 0.483 & 0.314 & 0.575 & 1.311 & 0.509 & 3.38 \\
\hline & Cytotoxicity & -0.358 & 0.65 & 0.303 & 0.582 & 0.699 & 0.195 & 2.5 \\
\hline & Immunoproteasome & 0.326 & 0.325 & 1.011 & 0.315 & 1.386 & 0.733 & 2.619 \\
\hline & Apoptosis & -0.132 & 0.261 & 0.254 & 0.614 & 0.877 & 0.525 & 1.463 \\
\hline & Inflamm. chemokines & 0.246 & 0.319 & 0.596 & 0.44 & 1.279 & 0.685 & 2.389 \\
\hline & MAGEs & -3.168 & 1.014 & 9.76 & 0.002 & 0.042 & 0.006 & 0.307 \\
\hline & IFN downstream & -1.159 & 0.362 & 10.255 & 0.001 & 0.314 & 0.154 & 0.638 \\
\hline & Myeloid inflammation & -0.047 & 0.183 & 0.067 & 0.795 & 0.954 & 0.667 & 1.364 \\
\hline & B cells & 0.114 & 0.389 & 0.085 & 0.77 & 1.12 & 0.522 & 2.403 \\
\hline & CD45 & -0.068 & 0.293 & 0.054 & 0.817 & 0.934 & 0.526 & 1.659 \\
\hline & $\mathrm{CD}^{+}$cells & -0.295 & 0.437 & 0.454 & 0.501 & 0.745 & 0.316 & 1.755 \\
\hline & CTLs & 0.505 & 0.852 & 0.351 & 0.554 & 1.657 & 0.312 & 8.805 \\
\hline & DCs & 1.574 & 0.831 & 3.589 & 0.058 & 4.827 & 0.947 & 24.601 \\
\hline & Exhausted $\mathrm{CD}^{+}{ }^{+}$cells & -0.487 & 0.497 & 0.96 & 0.327 & 0.614 & 0.232 & 1.628 \\
\hline & Macrophages & 0.222 & 0.295 & 0.57 & 0.45 & 1.249 & 0.701 & 2.225 \\
\hline & Mast cells & 0.066 & 0.123 & 0.292 & 0.589 & 1.069 & 0.84 & 1.359 \\
\hline & PMN & 0.004 & 0.176 & 0 & 0.984 & 1.004 & 0.711 & 1.417 \\
\hline & NK cells & -0.462 & 0.523 & 0.782 & 0.377 & 0.63 & 0.226 & 1.755 \\
\hline & T cells & -0.279 & 0.471 & 0.352 & 0.553 & 0.756 & 0.301 & 1.902 \\
\hline & Th1 & -0.004 & 0.478 & 0 & 0.993 & 0.996 & 0.39 & 2.541 \\
\hline & TIS & 1.728 & 0.982 & 3.1 & 0.078 & 5.63 & 0.822 & 38.548 \\
\hline & {$[E L N=A D V]$} & 0.039 & 0.365 & 0.012 & 0.914 & 1.04 & 0.509 & 2.127 \\
\hline & [ELN=FAV] & 1.492 & 0.287 & 26.969 & 0 & 4.445 & 2.531 & 7.804 \\
\hline & [ELN=INT] & $0^{\wedge}$ & . & . & . & . & . & \\
\hline NR & Intercept & -18.35 & 6.566 & 7.808 & 0.005 & & & \\
\hline & BM blasts & 0.001 & 0.006 & 0.05 & 0.823 & 1.001 & 0.989 & 1.014 \\
\hline & Age & 0.02 & 0.01 & 4.354 & 0.037 & 1.02 & 1.001 & 1.04 \\
\hline & Lymphoid cells & 1.185 & 1.557 & 0.579 & 0.447 & 3.271 & 0.155 & 69.157 \\
\hline & Myeloid cells & 0.262 & 0.481 & 0.297 & 0.586 & 1.3 & 0.506 & 3.338 \\
\hline & APM & 0.129 & 0.629 & 0.042 & 0.838 & 1.138 & 0.331 & 3.905 \\
\hline & MHC2 & -0.158 & 0.139 & 1.286 & 0.257 & 0.854 & 0.65 & 1.122 \\
\hline & IFN- $\gamma$ & -0.562 & 0.553 & 1.034 & 0.309 & 0.57 & 0.193 & 1.685 \\
\hline & Cytotoxicity & -0.4 & 0.715 & 0.313 & 0.576 & 0.67 & 0.165 & 2.721 \\
\hline & Immunoproteasome & 0.764 & 0.358 & 4.55 & 0.033 & 2.148 & 1.064 & 4.335 \\
\hline & Apoptosis & 0.135 & 0.289 & 0.219 & 0.64 & 1.145 & 0.65 & 2.016 \\
\hline & Inflamm. chemokines & 0.053 & 0.336 & 0.025 & 0.874 & 1.055 & 0.546 & 2.036 \\
\hline & MAGEs & -2.088 & 1.022 & 4.172 & 0.041 & 0.124 & 0.017 & 0.919 \\
\hline & IFN downstream & -0.437 & 0.368 & 1.414 & 0.234 & 0.646 & 0.314 & 1.328 \\
\hline & Myeloid inflammation & 0.337 & 0.183 & 3.417 & 0.065 & 1.401 & 0.98 & 2.004 \\
\hline
\end{tabular}




\begin{tabular}{|c|c|c|c|c|c|c|c|c|}
\hline B cells & 0.081 & 0.41 & 0.039 & & 0.844 & 1.084 & 0.485 & 2.422 \\
\hline CD45 & 0.121 & 0.312 & 0.149 & & 0.699 & 1.128 & 0.612 & 2.081 \\
\hline $\mathrm{CD}^{+}$cells & -0.522 & 0.47 & 1.234 & & 0.267 & 0.593 & 0.236 & 1.491 \\
\hline CTLs & 0.439 & 0.919 & 0.228 & & 0.633 & 1.551 & 0.256 & 9.384 \\
\hline DCs & 1.651 & 0.873 & 3.579 & & 0.059 & 5.215 & 0.942 & 28.861 \\
\hline Exhausted $\mathrm{CD} 8^{+}$cells & 0.456 & 0.528 & 0.744 & & 0.388 & 1.578 & 0.56 & 4.443 \\
\hline Macrophages & -0.176 & 0.335 & 0.277 & & 0.599 & 0.838 & 0.435 & 1.616 \\
\hline Mast cells & 0.328 & 0.131 & 6.279 & & 0.012 & 1.389 & 1.074 & 1.795 \\
\hline PMN & -0.156 & 0.197 & 0.623 & & 0.43 & 0.856 & 0.581 & 1.26 \\
\hline NK cells & -0.164 & 0.532 & 0.096 & & 0.757 & 0.848 & 0.299 & 2.406 \\
\hline T cells & -0.275 & 0.497 & 0.308 & & 0.579 & 0.759 & 0.287 & 2.009 \\
\hline Th1 & 0.018 & 0.476 & 0.001 & & 0.97 & 1.018 & 0.401 & 2.587 \\
\hline TIS & 0.331 & 1.034 & 0.102 & & 0.749 & 1.392 & 0.183 & 10.563 \\
\hline$[E L N=A D V]$ & 1.168 & 0.3 & 15.151 & & 0 & 3.217 & 1.786 & 5.794 \\
\hline$[\mathrm{ELN}=\mathrm{FAV}]$ & -0.619 & 0.362 & 2.917 & & 0.088 & 0.539 & 0.265 & 1.096 \\
\hline$[\mathrm{ELN}=\mathrm{INT}]$ & $0^{\wedge}$ & $\cdot$ & . & & 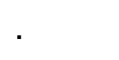 & & 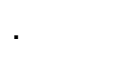 & 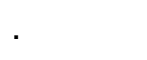 \\
\hline Intercept & 11.659 & 7.472 & 2.435 & 1 & 0.119 & & & \\
\hline BM blasts & -0.009 & 0.008 & 1.303 & 1 & 0.254 & 0.991 & 0.977 & 1.006 \\
\hline Age & 0.019 & 0.011 & 2.811 & 1 & 0.094 & 1.019 & 0.997 & 1.042 \\
\hline Lymphoid cells & 0.937 & 1.812 & 0.267 & 1 & 0.605 & 2.551 & 0.073 & 88.87 \\
\hline Myeloid cells & -0.233 & 0.561 & 0.173 & 1 & 0.677 & 0.792 & 0.264 & 2.377 \\
\hline APM & -1.241 & 0.726 & 2.922 & 1 & 0.087 & 0.289 & 0.07 & 1.199 \\
\hline $\mathrm{MHC2}$ & 0.118 & 0.179 & 0.435 & 1 & 0.509 & 1.126 & 0.792 & 1.6 \\
\hline IFN- $\gamma$ & -1.154 & 0.808 & 2.039 & 1 & 0.153 & 0.315 & 0.065 & 1.537 \\
\hline Cytotoxicity & 0.192 & 0.817 & 0.055 & 1 & 0.814 & 1.211 & 0.244 & 6.003 \\
\hline Immunoproteasome & 0.216 & 0.412 & 0.276 & 1 & 0.6 & 1.241 & 0.554 & 2.782 \\
\hline Apoptosis & -0.176 & 0.327 & 0.291 & 1 & 0.589 & 0.838 & 0.442 & 1.59 \\
\hline Inflamm. chemokines & 0.401 & 0.407 & 0.972 & 1 & 0.324 & 1.493 & 0.673 & 3.313 \\
\hline MAGEs & -1.897 & 1.283 & 2.186 & 1 & 0.139 & 0.15 & 0.012 & 1.855 \\
\hline IFN downstream & -0.852 & 0.482 & 3.123 & 1 & 0.077 & 0.426 & 0.166 & 1.097 \\
\hline Myeloid inflammation & 0.051 & 0.228 & 0.05 & 1 & 0.823 & 1.052 & 0.673 & 1.644 \\
\hline $\mathrm{B}$ cells & 0.102 & 0.481 & 0.045 & 1 & 0.833 & 1.107 & 0.431 & 2.843 \\
\hline CD45 & 0.141 & 0.387 & 0.132 & 1 & 0.716 & 1.151 & 0.539 & 2.458 \\
\hline $\mathrm{CD}^{+}$cells & -0.791 & 0.541 & 2.142 & 1 & 0.143 & 0.453 & 0.157 & 1.308 \\
\hline CTLs & -0.324 & 1.061 & 0.093 & 1 & 0.76 & 0.723 & 0.09 & 5.792 \\
\hline DCs & -0.484 & 1.101 & 0.193 & 1 & 0.66 & 0.616 & 0.071 & 5.336 \\
\hline Exhausted $\mathrm{CD}^{+}{ }^{+}$cells & -0.03 & 0.617 & 0.002 & 1 & 0.961 & 0.97 & 0.289 & 3.254 \\
\hline Macrophages & 0.21 & 0.384 & 0.298 & 1 & 0.585 & 1.233 & 0.581 & 2.617 \\
\hline Mast cells & -0.149 & 0.167 & 0.803 & 1 & 0.37 & 0.861 & 0.621 & 1.194 \\
\hline PMN & 0.13 & 0.221 & 0.348 & 1 & 0.555 & 1.139 & 0.739 & 1.755 \\
\hline NK cells & -0.334 & 0.652 & 0.262 & 1 & 0.609 & 0.716 & 0.199 & 2.571 \\
\hline T cells & 0.562 & 0.562 & 1.001 & 1 & 0.317 & 1.755 & 0.583 & 5.28 \\
\hline Th1 & 0.154 & 0.586 & 0.069 & 1 & 0.793 & 1.166 & 0.37 & 3.675 \\
\hline TIS & 2.164 & 1.301 & 2.769 & 1 & 0.096 & 8.707 & 0.681 & 111.402 \\
\hline$[E L N=A D V]$ & 0.209 & 0.414 & 0.255 & 1 & 0.614 & 1.232 & 0.548 & 2.773 \\
\hline [ELN=FAV] & 0.661 & 0.36 & 3.366 & 1 & 0.067 & 1.937 & 0.956 & 3.924 \\
\hline [ELN=INT] & $0^{\wedge}$ & & & 0 & & & & \\
\hline
\end{tabular}

* The reference category is: Relapse.

$\wedge$ This parameter is set to 0 because it is redundant. 
Legend: ELN cytogenetic risk, patient age, leukemia burden at diagnosis and immune gene signatures were selected as covariates (HOVON validation cohort; $n=618$ cases). HR = hazard ratio; DF = degrees of freedom; $\mathrm{EFS}=$ event-free survival; NR = no response to induction chemotherapy; $\mathrm{NRM}=$ non-relapse mortality; $\mathrm{BM}=$ bone marrow; TIS = tumor inflammation signature; MAGE = melanoma-associated antigen; IFN = interferon; $\mathrm{PMN}=$ polymorphonuclear cells; $\mathrm{DC}=$ dendritic cell; $\mathrm{CTL}=$ cytotoxic $\mathrm{T}$ lymphocyte; $\mathrm{APM}=$ antigen processing machinery; ELN = European Leukemia-Net; ADV = adverse cytogenetic risk; FAV = favorable cytogenetic risk; INT = intermediate cytogenetic risk. 
Table S9: Study participants in the CP-MGD006-01 clinical trial (NCT\#02152956) of flotetuzumab immunotherapy.

\begin{tabular}{|c|c|}
\hline Number of patients & 30 \\
\hline Number of BM samples & 49 \\
\hline Baseline & 30 \\
\hline End of cycle 1 & 19 \\
\hline Males/females (n) & $14 / 16$ \\
\hline Median age (range) & $57(27-74)$ \\
\hline \multicolumn{2}{|l|}{ Age (years) } \\
\hline $18-65$ & 23 \\
\hline $66-75$ & 7 \\
\hline \multicolumn{2}{|l|}{ Disease status at time of enrollment } \\
\hline Relapsed AML & 7 \\
\hline Chemotherapy-refractory AML & 23 \\
\hline Secondary AML & 12 \\
\hline \multicolumn{2}{|l|}{ Cytogenetic risk group (ELN; n) } \\
\hline Favorable & 6 \\
\hline Intermediate & 6 \\
\hline Adverse & 17 \\
\hline Unknown & 1 \\
\hline Prior lines of therapy ( $n$ and range) & $4(1-9)$ \\
\hline \multicolumn{2}{|l|}{ Response } \\
\hline $\mathrm{CR} / \mathrm{CRh} / \mathrm{CRi}$ & 8 \\
\hline PR & 1 \\
\hline $\mathrm{OB}$ & 3 \\
\hline $\mathrm{TF} / \mathrm{SD} / \mathrm{PD}$ & 18 \\
\hline
\end{tabular}

Legend: $\mathrm{ELN}=$ European Leukemia Net; $\mathrm{CR}=$ complete remission; $\mathrm{CRi}=\mathrm{CR}$ with incomplete hematologic recovery; $\mathrm{CRh}=\mathrm{CR}$ with partial hematologic recovery; $\mathrm{OB}=$ other benefit; $\mathrm{TF}=$ treatment failure; $\mathrm{SD}=$ stable disease; $\mathrm{PD}=$ progressive disease; $\mathrm{BM}=$ bone marrow; N.A. = not available. 
Table S10: Protein panel used for GeoMx Digital Spatial Profiling (DSP) of FFPE bone marrow biopsies (SAL cohort $[A]$ and flotetuzumab cohort $[B]$ ).

\begin{tabular}{|l|l|}
\hline Panel A & \\
\hline Housekeeping proteins & RPS6 \\
\hline Signal-to-noise ratio & Histone H3 \\
\hline & Mouse IgG control \\
\hline NK cells & IgG rabbit isotype control \\
\hline & CD56 \\
\hline T cells & GZMB \\
\hline & CD3 \\
\hline & CD4 \\
\hline & CD8A \\
\hline B cells & CD45RO \\
\hline & FoxP3 \\
\hline Monocytes/macrophages & CD19 \\
\hline & CD20 \\
\hline Immune checkpoints & CD14 \\
\hline & CD68 \\
\hline & B7-H3 (CD276) \\
\hline & VISTA (PD-1H) \\
\hline Signaling pathways & PD1 (CD279) \\
\hline & PD-L1 (CD274) \\
\hline & STAT3 \\
\hline & P-STAT3 \\
\hline Cancer drivers & AKT \\
\hline & P-AKT \\
\hline Other molecules & Bcl-2 \\
\hline & PTEN \\
\hline & $\beta$-catenin \\
\hline & Ki-67 \\
\hline & CD44 \\
\hline & CD45 \\
\hline & Pan-cytokeratin \\
\hline & $\beta 2-$ microglobulin \\
\hline & \\
\hline & \\
\hline & \\
\hline & \\
\hline & \\
\hline &
\end{tabular}

\begin{tabular}{|l|l|}
\hline Panel B & \\
\hline Housekeeping proteins & RPS6 \\
\hline & Histone H3 \\
\hline & GAPDH \\
\hline Isotype controls & Mouse IgG1 \\
\hline & Rabbit IgG1 \\
\hline & Mouse IgG2A \\
\hline Immune cell profiling core & $\beta 2-$ microglobulin \\
\hline & CD11c \\
\hline & CD20 \\
\hline & CD3 \\
\hline & CD4 \\
\hline
\end{tabular}




\begin{tabular}{|c|c|}
\hline & CD45 \\
\hline & CD56 \\
\hline & CD68 \\
\hline & CD8 \\
\hline & CTLA4 \\
\hline & GZMB \\
\hline & Ki-67 \\
\hline & PD1 (CD279) \\
\hline & PD-L1 (CD274) \\
\hline & Pan-cytokeratin \\
\hline & HLA-DR \\
\hline & SMA \\
\hline & Fibronectin \\
\hline IO drug target module & 4-1BB (CD137) \\
\hline & LAG3 \\
\hline & OX40L \\
\hline & Tim-3 \\
\hline & VISTA (PD-1H) \\
\hline & ARG1 \\
\hline & B7-H3 (CD276) \\
\hline & IDO1 \\
\hline & STING \\
\hline & GITR \\
\hline & 4-1BB (CD137) \\
\hline Immune activation status module & CD127 \\
\hline & CD25 \\
\hline & CD80 \\
\hline & $\mathrm{ICOS}$ \\
\hline & PD-L2 \\
\hline & CD40 \\
\hline & CD27 \\
\hline & CD44 \\
\hline & CD127 \\
\hline Immune cell typing module & CD45RO \\
\hline & FoxP3 \\
\hline & CD34 \\
\hline & CD66b \\
\hline & CD14 \\
\hline & FAP- $\alpha$ \\
\hline & CD163 \\
\hline & CD45RO \\
\hline Pan-tumor module & MART1 \\
\hline & NY-ESO-1 \\
\hline & S100B \\
\hline & $\mathrm{Bcl}-2$ \\
\hline & EpCAM \\
\hline & Her2/ErbB2 \\
\hline & PTEN \\
\hline & PR \\
\hline
\end{tabular}


Table S11: Antibody panel used for intracellular cytokine staining of bone marrow cell suspensions.

\begin{tabular}{|c|c|c|}
\hline Antigen & Clone & Fluorochrome \\
\hline \multicolumn{3}{|c|}{ Surface staining } \\
\hline CD3 & UCHT1 & PE-Dazzle 594 \\
\hline CD4 & RPA-T4 & Brilliant Violet 421 \\
\hline CD8 & RPA-T8 & APC-Fire 750 \\
\hline \multicolumn{3}{|c|}{ Intracellular staining } \\
\hline IL-2 & MQ1-17H12 & FITC \\
\hline IL-4 & 8D4-8 & $\mathrm{PE}$ \\
\hline IL-10 & JES3-9D7 & Alexa Fluor 647 \\
\hline IL-17 & BL168 & Per-CP-Cy5.5 \\
\hline TNF- & MAb11 & PE-Cy7 \\
\hline IFN- & B27 & Alexa Fluor 700 \\
\hline
\end{tabular}

